\title{
The Antarctic Impulsive Transient Antenna Ultra-high Energy Neutrino Detector Design, Performance, and Sensitivity for 2006-2007 Balloon Flight
}

\author{
P. W. Gorham ${ }^{1}$, P. Allison ${ }^{1}$, S. W. Barwick ${ }^{2}$, J. J. Beatty ${ }^{3}$, D. Z. Besson ${ }^{4}$, W. R. Binns ${ }^{5}$, C. Chen ${ }^{6}$, \\ P. Chen ${ }^{6,13}$, J. M. $\mathrm{Clem}^{7}$, A. Connolly ${ }^{8}$, P. F. Dowkontt ${ }^{5}$, M. A. DuVernois ${ }^{10}$, R. C. Field ${ }^{6}$, \\ D. Goldstein ${ }^{2}$, A. Goodhue ${ }^{9}$ C. Hast ${ }^{6}$, C. L. Hebert ${ }^{1}$, S. Hoover ${ }^{9}$, M. H. Israel ${ }^{5}$, J. Kowalski, ${ }^{1}$ \\ J. G. Learned ${ }^{1}$, K. M. Liewer ${ }^{11}$, J. T. Link ${ }^{1,13}$, E. Lusczek ${ }^{10}$, S. Matsuno ${ }^{1}$, B. C. Mercurio ${ }^{3}$, C. Miki ${ }^{1}$, \\ P. Miočinović ${ }^{1}$, J. Nam ${ }^{2,12}$, C. J. Naudet ${ }^{11}$, R. J. Nichol ${ }^{8}$, K. Palladino ${ }^{3}$, K. Reil ${ }^{6}$, A. Romero-Wolf ${ }^{1}$ \\ M. Rosen ${ }^{1}$, L. Ruckman ${ }^{1}$, D. Saltzberg ${ }^{9}$, D. Seckel ${ }^{7}$, G. S. Varner ${ }^{1}$, D. Walz ${ }^{6}$, Y. Wang ${ }^{12}$, F. Wu ${ }^{21}$ \\ (ANITA Collaboration) \\ 1 Dept. of Physics and Astronomy, Univ. of Hawaii, Manoa, \\ HI 96822. ${ }^{2}$ Univ. of California, Irvine CA 92697. ${ }^{3}$ Dept. of Physics, \\ Ohio State Univ., Columbus, $\mathrm{OH} 43210 .{ }^{4}$ Dept. of Physics and Astronomy, \\ Univ. of Kansas, Lawrence, KS 66045. ${ }^{5}$ Dept. of Physics, \\ Washington Univ. in St. Louis, MO 63130. ${ }^{6}$ Stanford Linear Accelerator Center, \\ Menlo Park, CA, 94025. ${ }^{7}$ Dept. of Physics, University of Delaware, \\ Newark, DE 19716. ${ }^{8}$ Dept. of Physics, University College London, London, \\ United Kingdom. ${ }^{9}$ Dept. of Physics and Astronomy, Univ. of California, \\ Los Angeles, CA 90095. ${ }^{10}$ School of Physics and Astronomy, Univ. of Minnesota, \\ Minneapolis, MN 55455. ${ }^{11}$ Jet Propulsion Laboratory, Pasadena, \\ CA 91109. ${ }^{12}$ Dept. of Physics, National Taiwan University, Taipei, \\ Taiwan. ${ }^{13}$ Currently at NASA Goddard Space Flight Center, Greenbelt, MD, 20771.
}

\begin{abstract}
We present a detailed report on the experimental details of the Antarctic Impulsive Transient Antenna (ANITA) long duration balloon payload, including the design philosophy and realization, physics simulations, performance of the instrument during its first Antarctic flight completed in January of 2007, and expectations for the limiting neutrino detection sensitivity. Neutrino physics results will be reported separately.
\end{abstract}

\section{INTRODUCTION}

Cosmic neutrinos of energy in the Exavolt and higher $\left(1 \mathrm{EeV}=10^{18} \mathrm{eV}\right)$ energy range, though as yet undetected, are expected to arise through a host of energetic acceleration and interaction processes at source locations throughout the universe. However, in only one of these sources-the distributed interactions of the ultra-high energy cosmic ray flux-does the combination of observational evidence and interaction physics lead to a strong requirement for resulting high energy neutrinos. Whatever the sources of the highest energy cosmic rays, their observed presence in the local universe, combined with the expectation that their sources occur widely throughout the universe at all epochs, leads to the conclusion that their interactions with the cosmic microwave background radiation (CMBR)- the so-called GZK process (after Greisen, Zatsepin, and Kuzmin [1])- must yield an associated cosmogenic neutrino flux, as first noted by Berezinsky and Zatsepin [2]. These neutrinos are often called the GZK neutrinos, as they arise from the same interactions of the ultra-high energy cosmic rays (UHECR) that cause the GZK cutoff, but they are perhaps more properly referred to as the BZ neutrinos.

In BZ neutrino production scenarios, current experimental UHECR measurements invariably point to the presence of an associated ultra-high energy neutrino flux. For UHECR above several times $10^{19} \mathrm{eV}$, intergalactic space is optically thick to UHECR propagation through the CMBR at a distance scale of several tens of Mpc. Each UHECR source at all epochs is thus subject to local conversion of its hadronic flux to secondary, lower energy particles over a distance scale of order $100 \mathrm{MPc}$ in the current epoch. Neutrinos are the only secondary particle that may freely propagate to cosmic distances, and the resulting neutrino flux at earth is thus related to the integral over the highest-energy cosmic ray history of the universe, to the earliest epoch at which they occur.

Although local sources may also contribute to the EeV-ZeV neutrino flux at earth, the bulk of the flux is generally believed to arise from a much wider spectral convolution, and will thus be imprinted with the cosmological source distribution in addition to effects from local sources. This leads to strong motivations to detect the BZ neutrino flux: first, it is required by standard model physics, and thus its absence could signal new physics beyond the standard model. Second, it is the only way to directly observe the UHECR source behavior over cosmic distance scales. Finally, once established, the spectrum and absolute flux of such neutrinos may afford a calibrated "test beam" for both particle 
physics and astrophysics experimentation, providing center-of-momentum energies on target nucleons of 100-1000 $\mathrm{TeV}$, an energy scale not likely to be reached by other methods in the near future.

The Antarctic Impulsive Transient Antenna was designed with the goal of measuring the BZ neutrino flux directly, or limiting it at a level which would provide compelling and useful constraints on the early UHECR source history. The BZ neutrino flux is potentially very low-of order 1 neutrino per square kilometer per week arriving over $2 \pi$ steradians is a typical estimate. This flux presents an extreme challenge to detection, since the low neutrino interaction cross section also means that any target volume will have an inherently low efficiency for converting any given neutrino. ANITA's methodology centers on observing the largest possible volume of the most transparent possible target material: Antarctic ice, which has been demonstrated to provide extremely low-loss transmission of radio signals through its bulk over much of the continent. ANITA then exploits the Askaryan effect [3], coherent, impulsive radio emission from the charge asymmetry in the electromagnetic component of a high energy particle cascade in a dielectric medium.

ANITA searches for cascades initiated by a primary neutrino interacting in the Antarctic ice sheet within its field of view from the Long-Duration Balloon (LDB) altitude of $35-37 \mathrm{~km}$. The observed area of ice from these altitudes is of order $1.5 \mathrm{M} \mathrm{km}^{2}$. Combining this with the electromagnetic field attenuation length of ice which is of order $1 \mathrm{~km}$ at ANITA's observation frequency range, ANITA is sensitive to a target volume of order 1-2 $\mathrm{M} \mathrm{km}^{3}$. The acceptance, however, is constrained by the fact that at any location within the target, the allowed solid angle of arrival for a neutrino to be detectable at the several-hundred-km average distance of the payload is a small fraction of a steradian. Folding in these constraints, the volumetric acceptance is still of order hundreds to thousands of $\mathrm{km}^{3}$ steradians over the range of energy overlap- $10^{18.5-20} \mathrm{eV}$-with the BZ neutrino spectrum. This large acceptance, while tempered by the limited exposure in time provided by a balloon flight, still yields the largest sensitivity of any experiment to date for BZ neutrinos. In this report we document the ANITA instrument and our estimates of its sensitivity and performance for the first flight of the payload, completed in January of 2007. A separate report will detail the results on the neutrino flux.

\section{THEORETICAL BASIS FOR ANITA METHODOLOGY.}

The concept of detecting high energy particles through the coherent radio emission from the cascade they produce can be traced back over 40 years to Askaryan [3], who argued persuasively for the presence of strong coherent radio emission from these cascades, and even suggested that any large volume of radio-transparent dielectric, such as an ice sheet, a geologic saltbed, or the lunar regolith could provide the target material for such interactions and radio emission. In fact all of these approaches are now being pursued [ [ , 5, 6].

Although significant early efforts were successful in detecting radio emission from high energy particle cascades in the earth's atmosphere [7], it is important to emphasize that the cascade radio emission that ANITA detects is unrelated to the primary mechanism for air shower radio emission. Particle cascades induced by neutrinos in Antarctic ice are very compact, consisting of a "plug" of relativistic charged particles several $\mathrm{cm}$ in diameter and $\sim 1 \mathrm{~cm}$ thick, which develops at the speed of light over a distance of several meters from the vertex of the neutrino interaction, before dissipating into residual ionization in the ice. The resulting radio emission is coherent Cherenkov radiation with a particularly clean and simple geometry, providing high information content in the detected pulses. In contrast, the radio emission from air showers is a complex phenomenon entangled with geomagnetic and near field effects. Attempts to understand and exploit this form of air shower emission for cosmic ray studies have been hampered by this complexity since its discovery in the mid-1960's, although this effort has seen a recent renaissance [? ].

Surprisingly little work was done on Askaryan's suggestions that solids such as ice could be important media for detection until the mid-1980's, when Gusev and Zheleznykh [10], and Markov \& Zheleznykh [11] revisited these ideas. More recently a host of investigators including Zheleznykh [12], Dagkesamansky \& Zheleznykh [13], Frichter, Ralston, \& McKay [17], Zas, Halzen, \& Stanev [14], Alvarez-Muñiz \& Zas [15], and Razzaque et. al [19] among others have taken up these suggestions and confirmed the basic results through more detailed analysis. Of equal importance, a set of experiments at the Stanford Linear Accelerator center have now clearly confirmed the effect and explored it in significant detail [6, 16, 20, 21]

\section{A. First Order Energy Threshold \& Sensitivity.}

To illustrate the methodology, we consider a specific example. The coherent radio Cherenkov emission in an electromagnetic $e^{+} e^{-}$cascade arises from the $\sim 20 \%$ electron excess in the shower, which is itself produced primarily 
by Compton scattering and positron annihilation in flight. Considering deep-inelastic scattering charged-current interactions of a high energy neutrino $v$ with a nucleon $N$, given generically by $v+N \rightarrow \ell^{ \pm}+X$, the charged lepton $\ell^{ \pm}$escapes while the hadronic debris $X$ leads to a hadronic cascade. If the initial neutrino has energy energy $E_{v}$, the resulting hadronic cascade energy will $E_{c}=y E_{v}$, where $y$ is the Bjorken inelasticity, with a mean of $\langle y\rangle \simeq 0.22$ at very high energies, and a very broad distribution. The average number of electrons and positrons $N_{e+e-}$ near total shower maximum is of order the cascade energy expressed in $\mathrm{GeV}$, or

$$
N_{e+e-} \simeq \frac{E_{c}}{1 \mathrm{GeV}} .
$$

Consider a case with $E_{\mathrm{v}}=10^{19} \mathrm{eV}$ and a slightly positive fluctuation above the mean giving $y=0.4$. This leads to $E_{c}=4 \times 10^{18} \mathrm{eV}$, giving $N_{e+e-} \sim 4 \times 10^{9}$. The radiating charge excess is then of order $N_{e x} \simeq 0.2 N_{e+e-}$. Singlecharged-particle Cherenkov radiation gives a total radiated energy, for tracklength $L$ over a frequency band from $v_{\min }$ to $\mathrm{v}_{\max }$, of:

$$
W_{t o t}=\left(\frac{\pi h}{c} \alpha\right) L\left(1-\frac{1}{n^{2} \beta^{2}}\right)\left(v_{\max }^{2}-v_{\min }^{2}\right)
$$

where $\alpha \simeq 1 / 137$ is the fine structure constant, $h$ and $c$ are Planck's constant and the speed of light, and $n$ and $\beta$ are the medium dielectric constant, and the particle velocity relative to $c$, respectively. For a collection of $N$ charged particles radiating coherently (e.g., with mean spacing small compared to the mean radiated wavelength), the total energy will be of order $W_{t o t}=N^{2} w$. In solid dielectrics with density comparable to ice or silica sand, the cascade particle bunch is compact, with transverse dimensions of several $\mathrm{cm}$, and longitudinal dimensions of order $1 \mathrm{~cm}$. Thus coherence will obtain up to several $\mathrm{GHz}$ or more.

For a $4 \times 10^{18} \mathrm{eV}$ cascade, $N_{e x} \simeq 8 \times 10^{8}$, and $L \simeq 6 \mathrm{~m}$ in the vicinity of shower maximum in a medium of density $\sim 0.9$ with $n \sim 1.8$ as in Antarctic ice. Taking the mean radio frequency to be $0.6 \mathrm{GHz}$ with an effective bandwidth of $600 \mathrm{MHz}$, the net radiated energy is $W_{\text {tot }}=10^{-7} \mathrm{~J}$. This energy is emitted into a restricted solid angle defined by the Cherenkov cone at an angle $\theta_{c}$ defined by $\cos \theta_{c}=(n \beta)^{-1}$, and a width determined (primarily from diffraction considerations) by $\Delta \theta_{c} \simeq c \sin \theta_{c} /(\bar{v} L)$. The implied total solid angle of emittance is $\Omega_{c} \simeq 2 \pi \Delta \theta_{c} \sin \theta_{c}=0.36 \mathrm{sr}$.

The pulse is produced by coherent superposition of the amplitudes of the Cherenkov radiation shock front, which yields extremely broadband spectral power over the specified frequency range. The intrinsic pulse width is less than $100 \mathrm{ps}$ [20], and this pulse thus excites a single temporal mode of the receiver, with characteristic time $\Delta t=(\Delta v)^{-1}$, or about $1.6 \mathrm{~ns}$ in our case here. Radio source intensity in radio astronomy is typically expressed in terms of the flux density Jansky (Jy), where $1 \mathrm{Jy}=10^{-26} \mathrm{~W} \mathrm{~m}^{-2} \mathrm{~Hz}^{-1}$. The energy per unit solid angle derived above, $W_{\text {tot }} / \Omega_{c}=$ $2.7 \times 10^{-7} \mathrm{~J} / \mathrm{sr}$ in a $600 \mathrm{MHz}$ bandwidth, produces an instantaneous peak flux density of $S_{c}=1.4 \times 10^{7} \mathrm{Jy}$ at the mean geometric distance $D=480 \mathrm{~km}$ of the ice in view, after accounting for the fact that at this distance the geometry constrains the Fresnel coefficient for transmission through the ice surface to $\sim 0.12$, since the radiation emerges from angles close to the total-internal-reflectance (TIR) angle.

The sensitivity of a radio antenna is determined by its collecting aperture and the thermal noise background, called the system temperature $T_{\text {sys. }}$. The RMS level of power fluctuations in this thermal noise, expressed in $\mathrm{W} \mathrm{m}^{-2} \mathrm{~Hz}^{-1}$, is given by

$$
\Delta S=\frac{k T_{\text {sys }}}{A_{\text {eff }} \sqrt{\Delta t \Delta v}} \mathrm{~W} \mathrm{~m}^{-2} \mathrm{~Hz}^{-1}
$$

where $k$ is Boltzmann's constant and $A_{\text {eff }}$ is the effective area of the antenna. Note that in our case, because the pulse is band-limited, the term $\sqrt{\Delta t \Delta v} \simeq 1$. For ANITA, a single on-board antenna has a frequency-averaged effective area of $0.2 \mathrm{~m}^{2}$. For observations of ice the system temperature is dominated by the ice thermal emissivity with $T_{s y s} \leq 320 \mathrm{~K}$, assuming $\sim 140 \mathrm{~K}$ receiver noise temperature. The implied RMS noise level is thus $\Delta S=2 \times 10^{6} \mathrm{Jy}$, giving a signalto-noise ratio of 6.3 in this case. These simple arguments show that the expected threshold for neutrino detection is of order $10^{19} \mathrm{eV}$ even to the edges of the observed area viewed by ANITA. In practice, events may be detected at lower energies due to fluctuations or interactions closer to the payload, but more detailed simulations of the energy-dependent acceptance of ANITA do not depart greatly from this first-order example. 


\section{INSTRUMENT DESIGN}

\section{A. Overview of Technical Approach}

As indicated by its acronym, ANITA is conceptually an antenna or antenna array optimized to detect impulsive RF events with a characteristic signature established by careful modeling and experimental measurements. The array of antennas should view most of the entire Antarctic ice sheet beneath the balloon, out to the horizon, to retain sensitivity to most of the potential ice volume available for neutrino event production. It should have the ability to trigger with high efficiency on events of interest, and should have the lowest feasible intrinsic noise levels in its receivers to maximize sensitivity. It should have broad radio spectral coverage and dual-polarization capability to improve its ability to identify the signals and reject the backgrounds. It must have immunity to transient or steady radio-frequency interference. It must have enough spatial resolution of the source of measured pulses to determine if they match expected signal sources, and to allow for first-order geolocation and subsequent sky mapping if the event is found to be consistent with a neutrino. It must make as many distinct and statistically independent measurements as possible of each impulse that triggers the system covering all available degrees of freedom (spatial, temporal, polarization, and spectral), because the number of potential neutrino events among these triggers may be close to zero, and this potential rarity of events demands that the information content of each measured event be maximized.

These guiding principles have led to a technical approach that centers around dual-polarization, broadband antenna clusters with overlapping fields-of-view, combined with a trigger system based on a heritage of RF impulse detection instruments, both space-based (the FORTE satellite [22, 23] ) and ground-based (the GLUE and RICE experiments [ 7 , 5]). The need for direction determination, combined with the constraints on usable radio frequency range dictated by ice parameters, leads to an overall geometry for both individual antennas and the entire array, that is governed by the requirements for radio pulse interferometry over the spectral band of interest.

The key challenges for ANITA are in the area of background rejection and management of electromagnetic interference (EMI). Impulsive interference events are likely to be primarily from anthropogenic sources, and in most cases do not mimic real cascade Cherenkov radio impulses because they lack many of the required properties such as polarization-, spectral-, and phase-coherence. A subset of impulsive anthropogenic interference, primarily from systems where spark gaps or rapid solid-state switching relays are employed, can produce events which are difficult to distinguish from events of interest to ANITA, and thus the task of pinpointing the origin of any impulsive event is of high importance to the final selection of neutrino candidates. If, after rejection of all impulsive events associated with any known current or prior human activity, there remains a class of events which are distributed across the integrated field of view of the payload and in time in a way that is inconsistent with human origin, we may then begin to consider this event class as containing neutrino candidates. Whether they survive with that designation will ultimately depend on our ability to exclude all other known possibilities.

In a previous balloon experiment [24], we found Antarctica to be relatively radio quiet at balloon altitudes once the payload leaves the vicinity of the largest bases. What continuous wave $(\mathrm{CW})$ interference is present can be managed with careful trigger configuration and threshold adjustment to servo-adjust for the temporary increases in narrow-band power that occasionally are seen. With regard to impulsive interference, we found triggers due to it to be relatively infrequent away from the main bases though even the smaller bases did occasionally produce bursts of triggers.

A less well-understood background may arise from ultra-high energy air showers which can produce a tail of radio emission out to ANITA frequencies, but these events, though they may produce triggers, are eliminated on the basis of their direction, arising from above the horizon, and their loss of coherence at VHF and UHF frequencies. However, in all cases above, ANITA may be presented with unexpected challenges.

\section{B. Background Interference Issues.}

Because ANITA operates with extremely high radio bandwidth over frequencies that are not reserved for scientific use, the problem of radio backgrounds, both anthropogenic and natural, is crucial to the development of a robust mission design. We have noted previously that the thermal noise power [61] provides the ultimate background limitation, for both impulsive and time-averaged measurements, in much the same way that photon noise provides one of the ultimate limits to optical imaging systems.

Electromagnetic interference may take different forms: near-sinusoidal "Carrier Wave" (CW) interference can have very high narrow-band power and saturate the system, or it can appear at a low level, sometimes as a composite of contributions from many bands, and effectively act to raise the aggregate system noise. Impulsive EMI often arises from electronic switching phenomena, and may trigger the system even if it cannot be mistaken for signals of interest, 
since the trigger should be as inclusive as possible. ANITA has only one chance per true neutrino event to detect and characterize the radio wavefront as it passes by the payload; thus it must be as efficient as possible at triggering on anything similar to the events of interest. In the end, it is the information content of a given triggered measurement that will determine the confidence with which we can ascribe it to a neutrino origin. This conclusion is the primary mission design driver for the type of payload and the number of antennas.

The design of the mission, payload, ballooncraft, and all ancillary instrumentation must therefore be evaluated in the light of whether it produces EMI, mitigates it, responds appropriately to it, or facilitates rejection of it. In the end, when all background interference has been rejected, what is left becomes the substance for ANITA science.

\section{Anthropogenic Backgrounds.}

Backgrounds from man-made sources do not in general pose a risk of being mistaken for the signals of scientific interest, unless they arise from locations where no human activity is previously known. As we will show later in this report, ANITA's angular reconstruction ability for terrestrial interference events gives accuracies of order 1 degree or better, enabling ground location of event sources to a level more than adequate to remove events that originate from known camps or anthropogenic sources. Human activity in Antarctica is highly controlled and positions and locations for all such activity are logged with high reliability during a season. However, man-made sources can still pose a significant risk of interfering with the operation of the instrument. Interference from man-made terrestrial or orbital sources is a ubiquitous problem in all of radio astronomy. In this respect ANITA faces a variety of potential interfering signals with various possible impacts on the data acquisition and analysis.

a. Satellite signals. Orbiting satellite transmitter power is generally low in the bands of interest. For example, the GPS constellation satellites at an altitude of $21000 \mathrm{~km}$, have transmit powers of order $50 \mathrm{~W}$ in the $1227 \mathrm{MHz}$ and 1575 $\mathrm{MHz}$ bands, with antenna gains of $11-13 \mathrm{dBi}$. The implied power at the earth's surface is $-127 \mathrm{~dB} \mathrm{~W} \mathrm{~m}^{-2}$ maximum in the $1227 \mathrm{MHz}$ band. The implied RMS noise voltage for ANITA, given the antenna's effective area at this frequency, is of order $0.7 \mu \mathrm{V}$, far below the RMS thermal noise voltage $(\sim 10-15 \mu \mathrm{V}$ RMS $)$ referenced to the receiver inputs. Current satellite systems do not typically operate in ANITA's band, however there are some legacy systems that can produce detectable power within ANITA's band. As we will discuss in a later section, ANITA has encountered some satellite interference in the $200-300 \mathrm{MHz}$ range, but it has not caused significant performance degradation to date.

Satellites do not in general intentionally produce nanosecond-scale impulsive signals; however, such signals may be produced by solid-state relay or actuator activity on a satellite that is changing its configuration. Such signals would appear to come from above the horizon, but might also show up in reflection off the ice surface. In this latter case, the Fresnel coefficient for such a reflection will in general signficantly boost the horizontal polarization of such a reflection, and this characteristic provides a strong discriminator, if the initial above-the-horizon impulse was for some reason not detected.

b. Terrestrial signals. The primary risk for terrestrial signals is not that they trigger the system. Terrestrial sources often do produce significant impulsive interference, and will trigger our system at significant rates anytime the payload is within view of such anthropogenic sources. However, such triggers are easily selected against in postanalysis since their directions can be precisely associated with known sources in Antarctica. The greater issue for ANITA occurs if there is a strong transmitter in the field of view which saturates the LNA, causing its gain to decrease so that the sensitivity in that antenna is lost. The present LNA design tolerates up to about $1 \mathrm{dBm}$ output before saturation, with an input stage gain of $36 \mathrm{~dB}$. Thus a signal of $0.25 \mu \mathrm{W}$ coupled into the antenna would pose a risk of saturation and temporary loss of sensitivity.

Since the antenna effective area is of order $0.6 \mathrm{~m}^{2}$ at the low end of the band, ANITA therefore tolerates up to a 0.2 MW in-band transmitter at or near the horizon, or a several $\mathrm{kW}$ in-band transmitter near the nadir, accounting for the off-axis response of the ANITA antennas. Most of the higher power radar and other transmitters in use in Antarctica are primarily at the South Pole and McMurdo stations. Such systems did reduce our sensitivity when the payload was in close proximity to McMurdo station, and to a lesser degree, when in view of the South Pole station.

\section{Other possible backgrounds.}

a. Lightning. Lightning is known to produce intense bursts of electromagnetic energy, but these have a spectrum that falls steeply with frequency, with very little power extending into the UHF and microwave regimes. Although lightning does occur over the Southern Ocean [25, 26], it is unknown on the Antarctic continent. We do not expect lightning to comprise a significant background to ANITA. 
b. Cosmic Ray Air Shower backgrounds. Cosmic ray extensive air showers (EAS) at EeV energies also produce an electromagnetic pulse, known from observations since the late 1960's. The dominant RF emission comes from synchrotron radiation in the geomagnetic field. This emission is coherent below about $100 \mathrm{MHz}$, transitioning to partial coherence above about $200 \mathrm{MHz}$ in the ANITA band. Although there has been a recent increase in activity to measure the radio characteristics of EAS events in the coherent regime below $100 \mathrm{MHz}$ [27], there is still little reliable information regarding the partially coherent regime where ANITA is sensitive to such events, although in fact several of the early detections of such events were at $500 \mathrm{MHz}$ [28]. The radio emission from EAS is highly beamed, so the acceptance for such events is naturally suppressed by geometry. They are also expected to have a steeply falling radio spectral signature, and thus an inverted spectrum compared to events originating from the Askaryan process, which has an intrinsic rising spectrum over the frequency region that coherence obtains, and a slow plateau and decline above those frequencies.

ANITA may detect such events either by direct signals or reflected signals off the ice surface, in a manner similar to that mentioned above for posible impulses from satellites. The EAS signals are known to be linearly polarized, with the plane of polarization determined by the local geomagnetic field direction. Since the field is largely vertical in the polar regions, there is a tendency for the EAS radio emission to be horizontally polarized for air showers with large zenith angles. ANITA's field-of-view, which has maximum sensitivity near the horizon, thus favors EAS events with these large zenith angles. Such events when observed directly arrive from angles above the horizon, but under the right circumstances they may also be seen in reflection, thus appearing to originate from below the horizon. They might thus be confused with neutrino-like events originating from under the ice, if their radio-spectral and polarization signature was not considered. In an appendix we will address this possible physics background and show why it is straightforward to separate it from the events of interest.

\section{CSBF Support Instrumentation Package.}

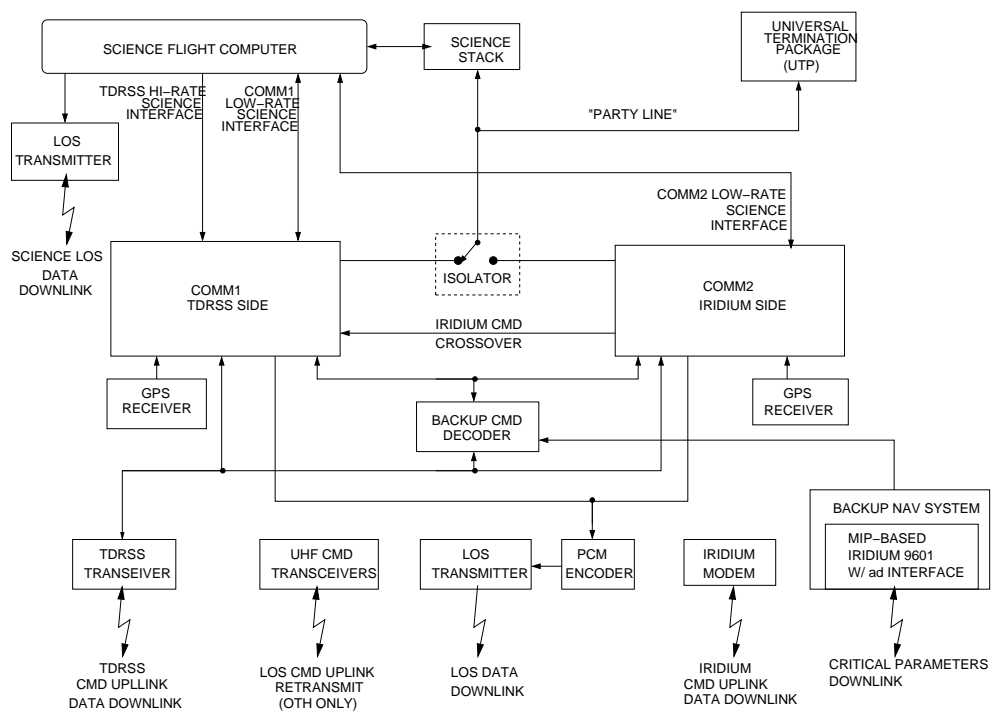

FIG. 1: Block diagram of the NSBF SIP.

Support for NASA long-duration balloon payload launches and in-flight services is provided through the staff of the Columbia Scientific Balloon Facility (CSBF), based in Palestine, Texas, USA. CSBF has developed a ballooncraft Support Instrument Package (SIP), an integrated suite of computers, sensors, actuators, relays, transmitters, and antennas, for use with all LDB science instruments. The CSBF SIP is controlled by a pair of independent flight computers which handle science telemetry, balloon operations, navigation, ballast control and the final termination and descent of the payload. A system diagram of the SIP is provided in Figure 1. A Science Stack, a configurable set of block modules, is also available as an option to the SIP providing such functions as a simple science flight computer, analog-to-digital conversion, and open-collector command outputs for additional instrument command and control. 
The SIP also provides the telemetry link between the ANITA flight computer and data acquisition system and ground based operations. Data from the ANITA computer is sent over serial lines to the SIP package which handles routing and transmission over line-of-sight (LOS), Tracking and Data Relay Satellite System (TDRSS), and IRIDIUM communication pathways. ANITA utilizes the NSBF SIP Science Stack to provide the ability to command the flight CPU system off and on and reboot the computer during flight.

With regard to computational resources of the SIP, these are designed to fulfill existing LDB requirements, including preserving a full archive of all telemetered data that is passed through the SIP from the science instrument. This function thus provides an additional redundant copy of the telemetered data that can be used if there is telemetry loss or corruption.

One important characteristic of the SIP relevant to ANITA is that it is not highly shielded from producing local EMI, at least at the extremely low level required for compatibility with ANITA science goals. Of necessity the SIP was thus enclosed in an external Faraday housing, with connectors, and penetrators designed in a manner similar to what was done for the ANITA primary electronics instrumentation.

\section{Gondola Structure.}
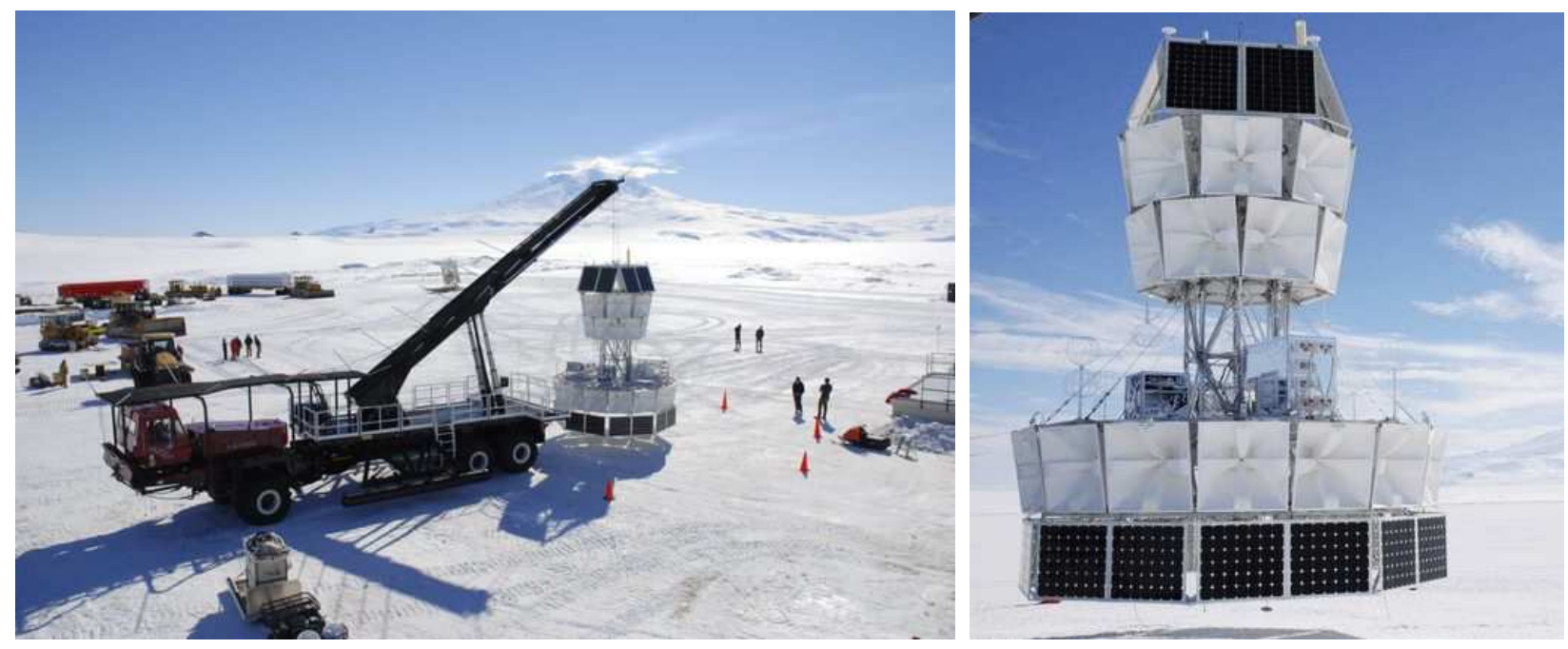

FIG. 2: ANITA payload in flight-ready configuration with launch vehicle.

The gondola structure consists primarily of an aircraft-grade aluminum alloy frame. A matrix of tubular components is pinned together via a combination of socket joints, tongue and clevis joints, and quarter-turn cam-lock fasteners. Views just prior to the launch of the payload show the structural elements of the gondola in Fig. 2. The frame is based on octagonal symmetry where eight vertical members, plus cross bracing, provide an internal backbone that allows for the attachment of spoke-like trusses to which the horn antennas fasten. Three ring-shaped clusters of quad-ridged horn antennas constitute the primary ANITA sensors. The top two antenna clusters have eight antennas each. Positioned around the perimeter of the base is a sixteen horn cluster. All of the eight horn clusters have a $45^{\circ}$ azimuthal offset angle between adjacent antennas, with a $22.5^{\circ}$ azimuthal offset between the top two rings. The antennas in the sixteen horn ring are offset from each other by $22.5^{\circ}$. All of the antennas in the upper two eight horn rings and the ones in the sixteen horn ring are canted down $10^{\circ}$ below horizontal to optimize their sensitivity, based on Monte Carlo studies of the effects of the tapering of the antenna beam when convolved with the neutrino arrival directions and energy spectrum.

The nearly circular plane that is established by the sixteen antenna ring, near the base of the gondola, provides a large deck area for most of the other payload components. This region is covered by lightweight panels made of dacron sailcloth on the topside and a reflective layer on the underside to maintain thermal balance. The ANITA electronics housing, the NASA/CSBF SIP, and the battery packs are mounted on the structural ribs of the deck. Most external metallic structure is painted white to avoid overheating in the continuous sunlight, and critical components such as the instrument housings and receivers are covered with silver-backed teflon-coated tape to provide high reflective rejection of solar radiation and high emissivity for internal heat dissipation. 


\section{E. Power subsystem.}

The ANITA power system is composed of a photovoltaic (PV) array, a charge controller, batteries, relays, and DCto-DC converters. The PV array is an omni-directional array consisting of eight panels configured in an octagon, with the panels hanging vertically (see instrument figure). Although PV panels flown on high-altitude balloons are typically oriented at $\sim 23^{\circ}$ to the horizontal, in Antarctica, the large solar albedo from the ice results in more irradiance incident on the panels (for most conditions) if they hang vertically. Each panel consists of 84 solar cells electrically connected in series. They were mounted on frames made of aircraft-grade spruce wood with a coarse webbing (Shearweave style1000-P02) stretched on the frames.

The PV arrays were designed and fabricated by SunCat Solar. The solar cells used were Sunpower A-300 cells with a rated efficiency of $21.5 \%$ and dimensions $12.5 \mathrm{~cm}$ square and thickness $260 \mathrm{um}$. Bypass diodes were placed in parallel with successive groups of 12 cells within a panel (7-diodes/panel) to mitigate the effect of a possible single cell open circuit failure during flight. Additionally, a blocking diode was placed between each panel output and the charge controller to prevent cross-charging of panels with different output voltages resulting from different illuminations and temperatures. To reduce Fresnel reflection losses for high-refractive index silicon ( $\mathrm{n}=3.46$ at $700 \mathrm{~nm})$, the silicon cells had two anti-reflective (AR) coatings applied. An AR coating with refractive index $n=1.92$ was applied by the solar cell manufacturers. Additionally, during fabrication of the panels by SunCat Solar, a second AR coating with refractive index 1.47 was applied. This results in calculated Fresnel losses of $13-14 \%$ for incidence angles from 0 to $40^{\circ}$.

The maximum power point (MPP) voltage and current generated by these cells under standard conditions (STC) are $0.560 \mathrm{~V}$ and $5.54 \mathrm{~A}$ respectively. However, the actual $\mathrm{V}$ and I vary considerably depending upon the irradiance and cell temperature. The single-cell temperature coefficient for the voltage is $-1.9 \mathrm{mV} / \mathrm{C}$. PV panel temperatures varied over the range of $-10 \mathrm{C}$ to $+95 \mathrm{C}$, depending upon the irradiance incident upon the cells. The temperatures were measured by semiconductor temp sensors (AD590) glued to the back of cells. PV array circuit components (diodes) also introduce losses in the output voltage and power. The actual measured PV voltage input to the charge controller during the flight ranged from 42.5 to $47 \mathrm{~V}$ (in good agreement with estimates using the cell temperature and temperature coefficient) and the current was about 9 A giving a total power of $400 \mathrm{~W}$.

The omni-directional array is inherently an unbalanced system; i.e. the irradiance incident on each panel differs. For a given orientation of the gondola, some panels are directly irradiated by sunlight plus solar albedo from the ice and others are irradiated only indirectly from solar albedo. Additionally, for those that are directly irradiated, the solar incidence angle is different. This results in individual panels that generate very different currents at any given time. Because of the differing temperatures, the individual panels also have significantly different output voltages (the voltage differences are small compared to the current differences) that feed into the charge controller. As mentioned above, the blocking diodes prevent cross-charging of panels generating different voltages.

When using an unbalanced array, to achieve the maximum power output, it is important to use a charge controller that senses and operates at the actual MPP as opposed to one that operates at a constant offset voltage from the array open-circuit voltage. We used an Outback MX-60 charge controller to supply power to the ANITA instrument. Conductive heat sinks were installed on the power FETs and transistors and the heat was conducted to the instrument radiator plate. We operated in the $24 \mathrm{~V}$ mode and flew nine pairs of $12 \mathrm{~V}$ Panasonic LC-X1220P (20 AH) lead acid batteries that were charged by the charge controller and would have provided 12 hours of power in case of PV array failure.

The Instrument Power box consisted of the MX-60 charge controller, solid-state power relays, and Vicor DC/DC converters for the external radio-frequency conditional module (RFCM) amplifiers. The main power relays for the cPCI crate were controlled by discrete commands from the SIP. All other solid-state relays were controlled by the CPU, either under software control or by commands from the ground. The DC/DC box consisted of Vicor DC/DC converters which provided the $+5,+12,-12,+3.3,+1.5$, and 5 voltages required by the cPCI crate and peripherals. All voltages and currents were read by the housekeeping system.

\section{F. Radio Frequency subsystem}

\section{Antennas.}

Figure 2 shows the ANITA payload configuration just prior to launch in late 2006 at Williams Field, Antarctica. The individual horns are a custom design produced for ANITA by Seavey Engineering, Inc., now a subsidiary of Antenna Research Associates, Inc. These horns are the primary ANITA antennas, and may be thought of as a flared quad-ridged waveguide section; the back of the horn does in fact terminate in a short section of waveguide. The dimension of the 

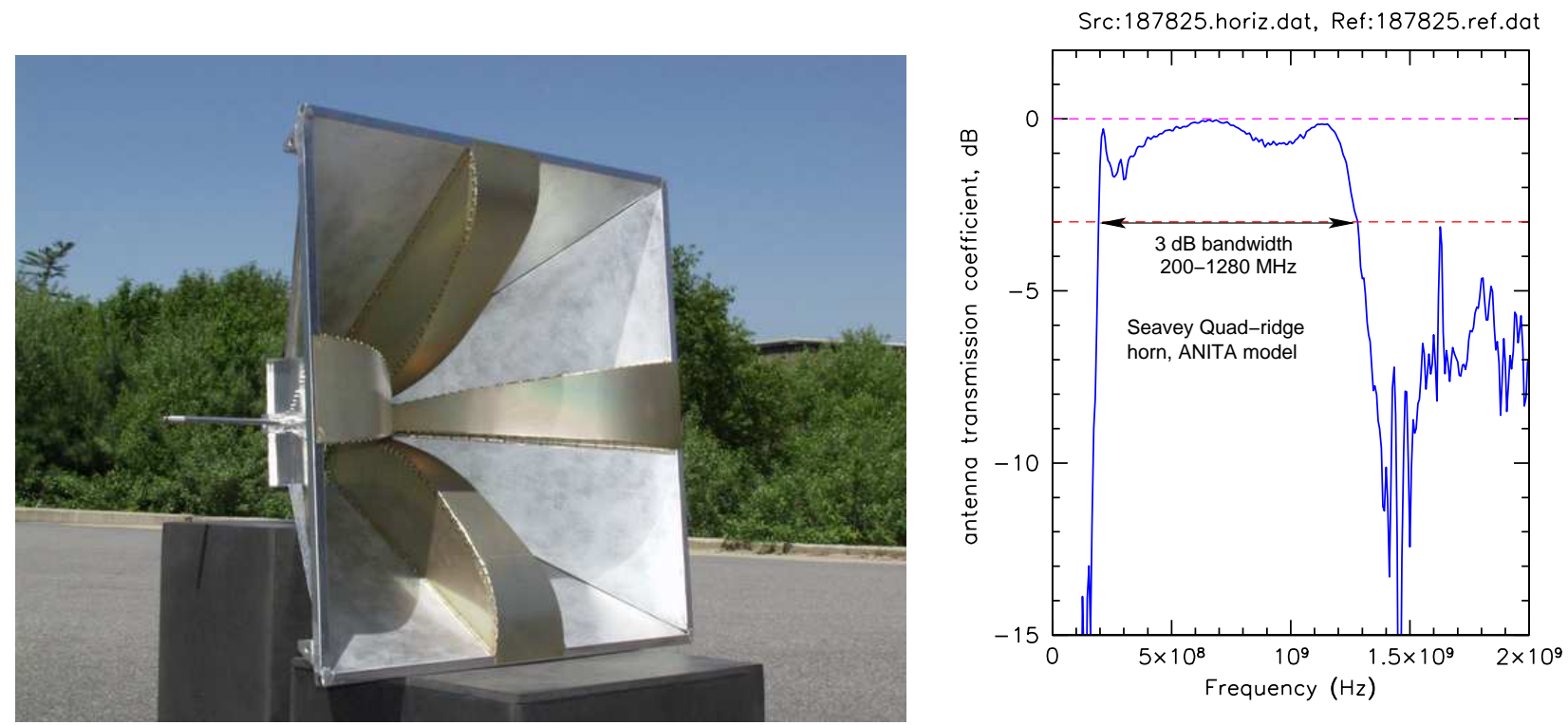

FIG. 3: Left: A photograph of an ANITA quad-ridged dual-polarization horn. Right: Typical transmission coefficient for signals into the quad-ridged horn as a function of radio frequency.

mouth is of order $0.8 \mathrm{~m}$ across, and the horns can be close-packed with minimal disturbance of the beam response since the fringing fields outside the mouth of the horn are small. Figure 3 shows an individual antenna prior to painting, and a corresponding typical transmission curve indicating the efficiency for coupling power into the antenna, as a function of radio frequency.

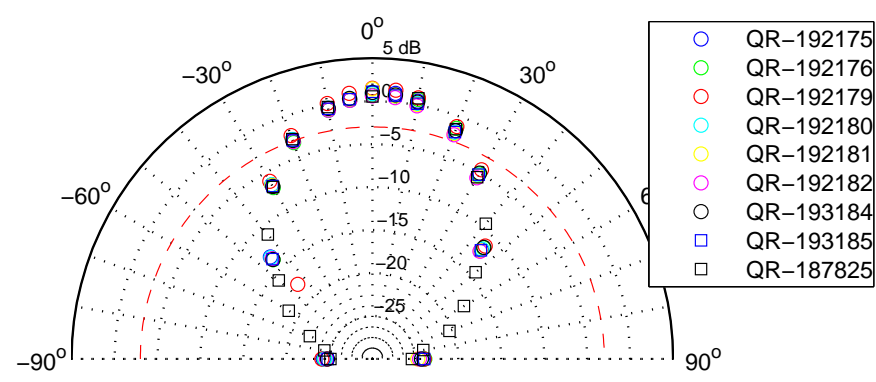

Vertical $\mathrm{H}$-plane mean relative gain

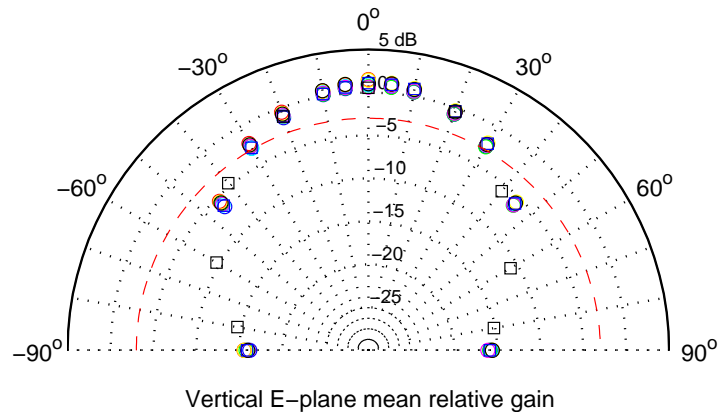

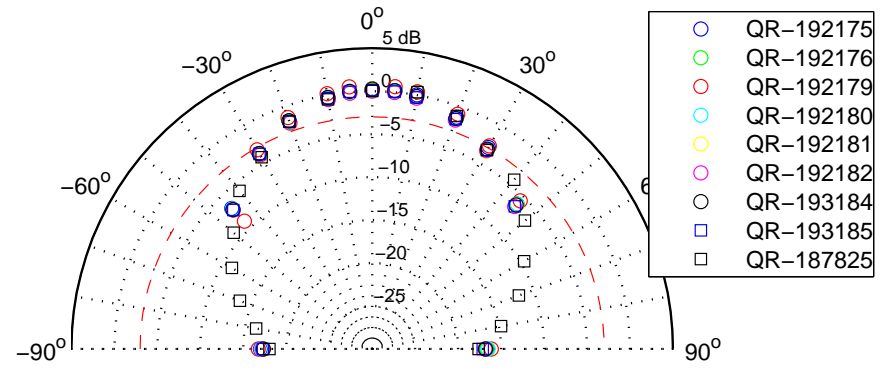

Horizontal E-plane mean relative gain

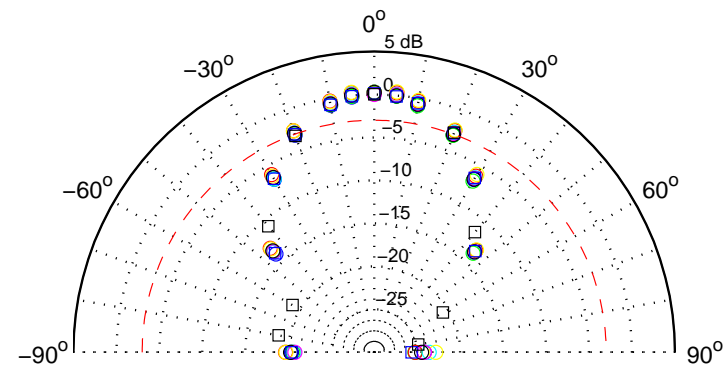

Horizontal $\mathrm{H}$-plane mean relative gain

FIG. 4: Left: Antenna vertical-polarization relative directivity in $\mathrm{dB}$ relative to the peak gain for both E and H-planes. Right: the same quantities for the horizontal polarization. Nine different antennas are shown. Gain is frequency-averaged for a flat-spectrum impulse across the band for different angles in 
The average full-width-at-half-maximum (FWHM) beamwidth of the antennas is about $45^{\circ}$ with a corresponding directivity gain (the ratio of $4 \pi$ to the main beam solid angle) of approximately $10 \mathrm{dBi}$ average across the band. Fig. 4 illustrates this for nine different ANITA antennas, showing the frequency-averaged response relative to peak response along the principal antenna planes (E-plane and H-plane for both polarizations) as a function of angle. The choice of beam pattern for these antennas also determined the $22.5^{\circ}$ angular offsets in azimuth, as this was chosen to provide good overlap between the response of adjacent antennas, but still maintaining reasonable directivity for determination of source locations.

By arranging an azimuthally symmetric array of 2 cluster groups of $8+8$ (upper) and 16 (lower) antennas, each with a downward cant of about $10^{\circ}$, we achieve complete coverage of the horizon down to within $40^{\circ}$ of the nadir, virtually all of the observable ice area. The antenna beams in this configuration overlap within their $3 \mathrm{~dB}$ points, giving redundant coverage in the horizontal plane. The $\sim 3 \mathrm{~m}$ separation between the upper and lower clusters of 16 antennas provides a vertical baseline for establishing pulse direction in elevation angle. Because the pulse from a cascade is known to be highly linearly-polarized, we convert the two linear polarizations of the antenna into dual circular polarizations using standard $90^{\circ}$ hybrid phase-shifting combiners. This is done for two reasons: first, a linearly polarized pulse will produce equal amplitudes in both circular polarizations, and thus some background rejection is gained by accepting only linearly-polarized signals; and second, the use of circular polarizations removes any bias in the trigger toward horizontal or vertically polarized impulses.
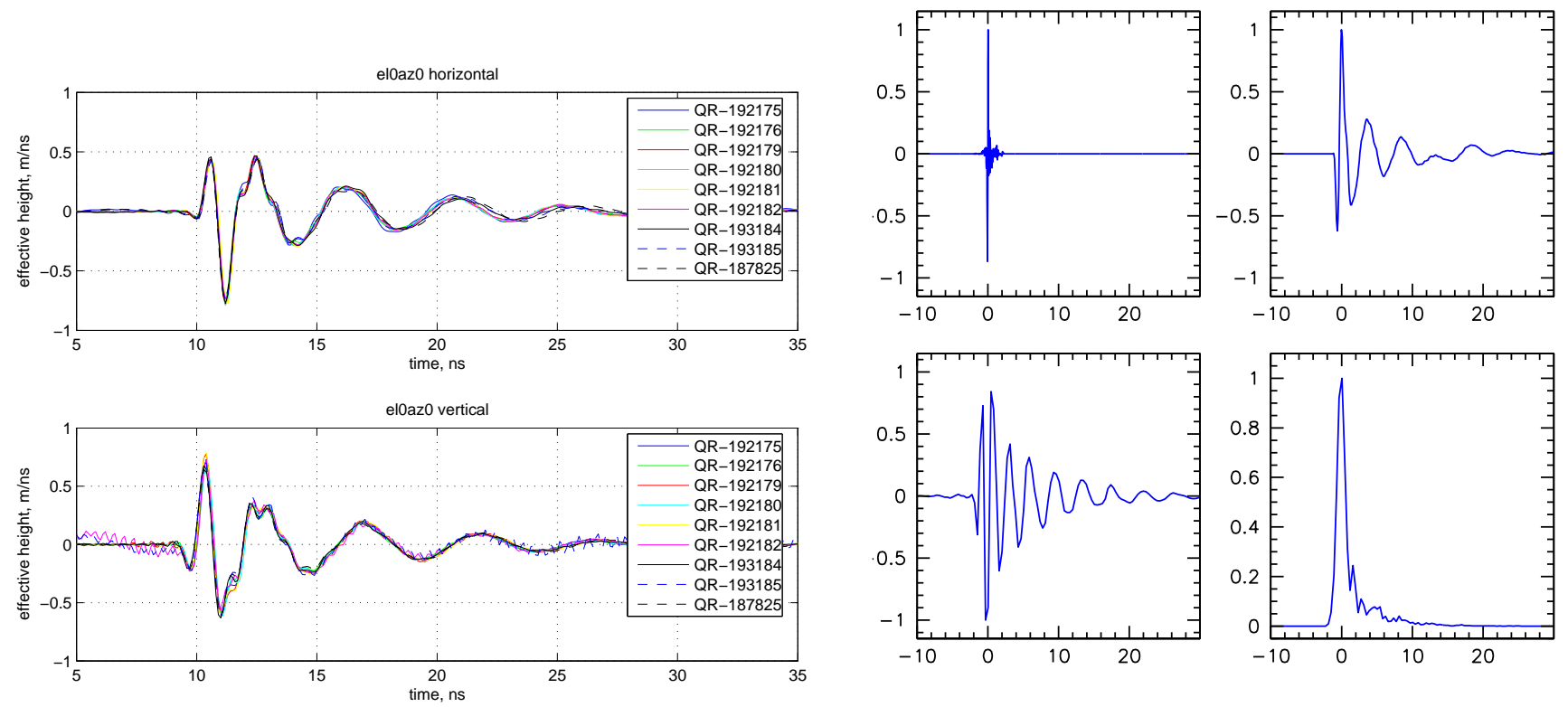

FIG. 5: Left: Antenna impulse response as measured for nine ANITA antennas, here in units that also give the instantaneous effective height [20]. Right: ANITA impulse response as it appears various stages of the signal chain.

Because ANITA's sensitivity to neutrino events depends crucially on its ability to trigger on impulses that rise above the intrinsically impulsive thermal noise floor, ANITA's antennas and receiving system must preserve the narrow impulsive nature of any signal that arrives at the antenna. Fig. 5 shows the measured behavior of the system impulse response at various stages. On the left, we show details of the measured impulse response of nine of the flight antennas, in units that give the instantaneous effective height $h_{e f f}(t)$. The actual voltage time response $\mathcal{V}(t)$ at the antenna terminals, assuming they are attached to a matched load, is then just the convolution of this function with the incident field $\mathcal{E}(t)$ :

$$
\mathcal{V}(t)=\frac{1}{2} \mathcal{E}(t) \otimes h_{e f f}(t)
$$

where the convolution operator is indicated by the symbol $\otimes$. This equation can also be expressed as an equivalent frequency domain form, though in that case the quantities are in general complex.

On the right, we show the evolution of an Askaryan impulse through the ANITA system. The initial Askaryan impulse (a) is completely unresolved by the ANITA system, since its intrinsic width is of order 100 ps (reference [20] 
provides the actual measured data that form the basis for this). The horn antenna response (b) includes group delay at the edges of the frequency band which leads to the low-frequency tail, and such group delay variation is more pronounced after the system bandpass filters are applied (c). However, the voltage envelope is slightly misleading, as the total power (or intensity) response (d) of the system is still confined to $1.5 \mathrm{~ns}$ FWHM.

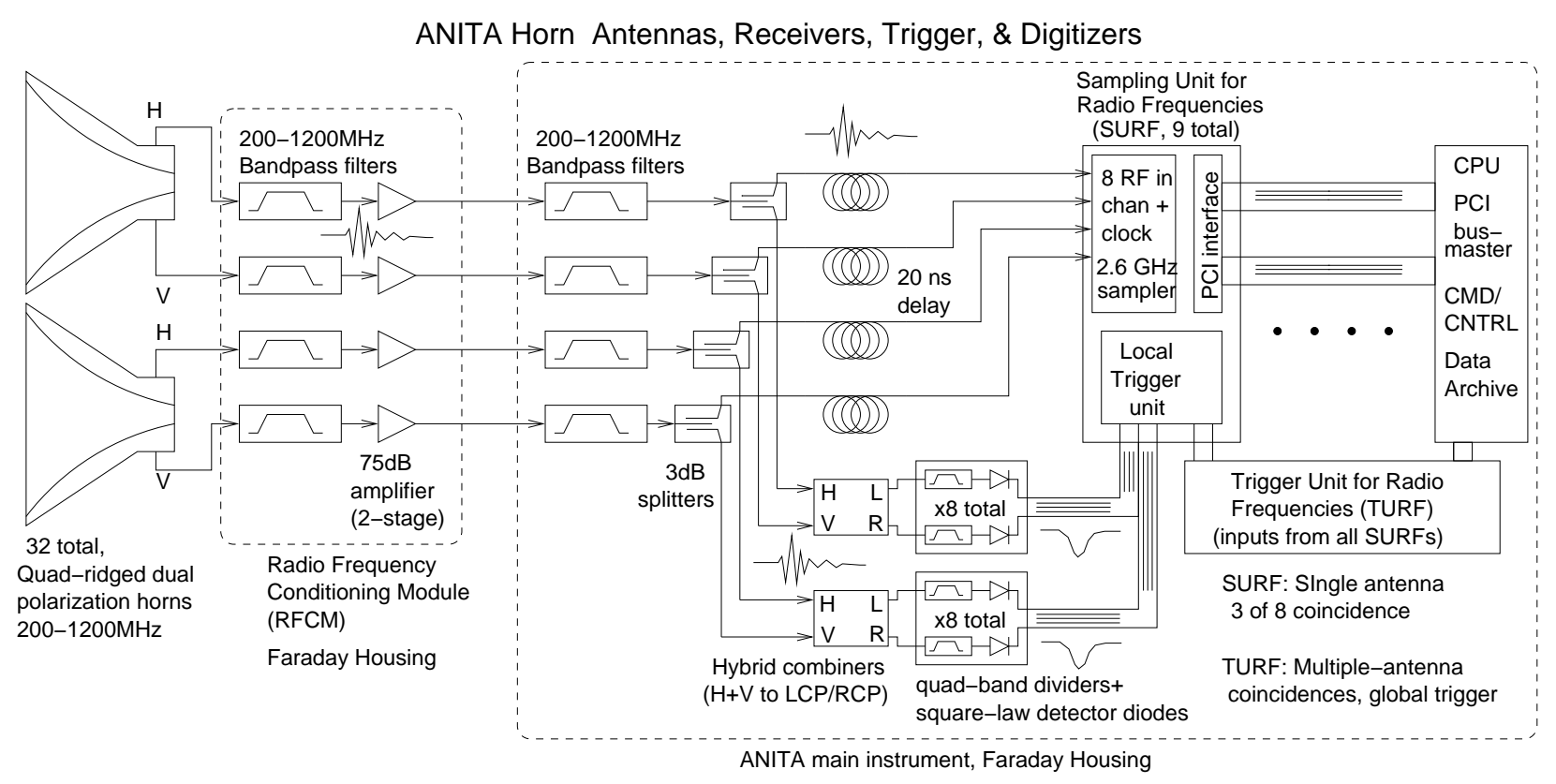

FIG. 6: Block diagram of the primary RF subsystems for ANITA.

\section{Receivers.}

The RF front end for ANITA consists of a bandpass filter, followed by a low-noise-amplifier (LNA)/power limiter combination, then followed by a 2 nd stage booster amplifier. An example of one of the receivers is shown in Fig. 7(left), with its enclosure cover removed to show the internal components. These elements are all in close proximity to the horn antennas to ensure no transfer losses through cables, and are enclosed in a Faraday box for additional EMI immunity. Once the signals are boosted by the 2nd stage amplifier, they are transmitted via coaxial cable to the receiving section of the trigger and digitizer, which is contained in a large shielded Faraday enclosure. Once the signals arrive at this location, a second bandpass filter is applied to remove the out-of-band noise from the LNA, and the signals are then ready for insertion into the trigger/digitizer system.

Figure 7(right) shows a composite overlay of measurements of the total gains and noise temperature for all ANITA channels, including cable attenuation losses up to the inputs of the digitizers. The gain slope is dominated by the intrinsic amplifier response. The noise temperature arises from the LNAs, with about $90 \mathrm{~K}$ intrinsic amplifier noise, combined with the front-end bandpass filter, which creates most of the additional $\sim 50 \mathrm{~K}$ due to emissivity along the signal path. The combined average noise temperature of $\left\langle T_{\text {sys }}\right\rangle \simeq 140 \mathrm{~K}$ was found to contribute at most about $40-45 \%$ of the total noise while at float, as we describe in a later section. Several of the receivers also had a coupler section added to them to allow for insertion of a calibration pulse into their respective antennas; these have a higher noise figure, but these channels were not used for the primary signal triggering. 

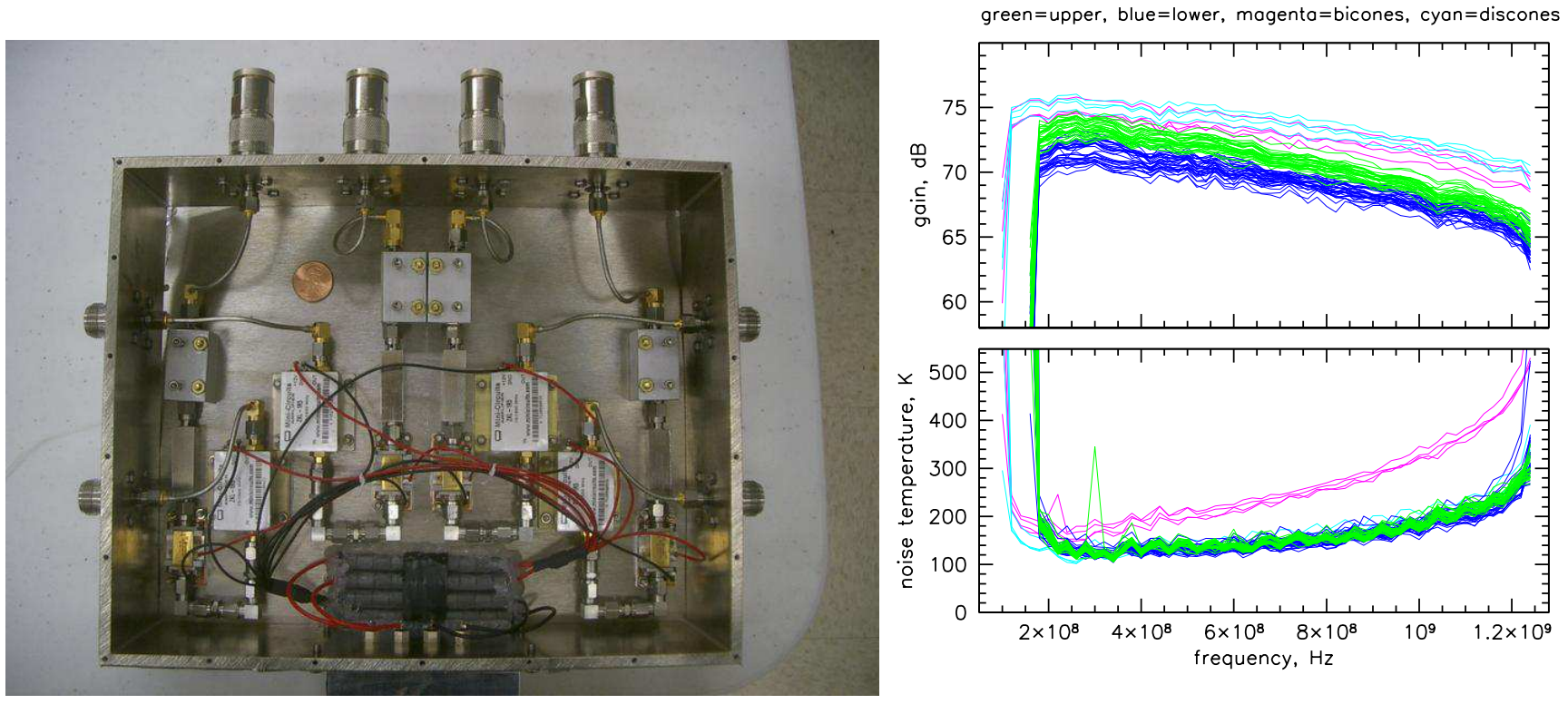

FIG. 7: Left: Photo Layout of ANITA receiver module. Right: Gain and noise measurements for all ANITA receivers receivers.

\section{G. Trigger and Digitizer Subsystem}

\section{Trigger System.}

In a long-duration balloon flight, primary power is solar which places tight restrictions on the payload power budget. Just as severe is the need to eliminate the heat generated by the payload electronics, which places practical limits of a $\mathrm{kW}$ or less on the entire payload instrumentation. ANITA seeks to digitize a large number of radio-frequency channels at rates of at least Nyquist frequencies for a bandwidth that extends up to $1.2 \mathrm{GHz}$, implying Nyquist sampling of at least 2.4 Gsamples/second. Commercial digitizers that run continuously at such rates are generally high-power devices, typically 5 watts per channel at the time of ANITA electronics development. For 80 channels, the required power for only the digitizers alone would be several hundred Watts, and the downstream electronics then required to parse and decimate the huge data rate (several Terabits per second) would use a comparable amount of power. Folding in the requirements for amplifiers and other analog and digital power needs, the payload power budget was not viable using commercial digitizers.

To make this problem tractable within the power, space, and weight budget of an LDB payload, we elected to develop a separate analog trigger system which would detect the presence of an incoming plane-wave impulse, then only digitize a time window around this pre-detected signal. The as-built design for the low-powered RF trigger and digitization electronics is summarized in Table 1 . A divide-and-conquer strategy to address the power and performance issues raised by these specifications is shown in Fig. 8.

a. Triggering at Thermal Noise Levels Actual neutrino signals are not expected to be observed at a rate greater than of order several per hour at most, if all previous bounds are saturated. However, to avoid creating too restrictive a trigger condition, the trigger was designed not to depend on exact time-alignment (or phase) of the incoming signal, over a time window of order $10 \mathrm{~ns}$. This allows accidentals from random thermal noise to also trigger the system, so that a continuous stream of events would be recorded, allowing a continuous sampling of the instrument health. Since the thermal noise floor in radio measurements is very well defined by the overall system temperature, this ensures that the sensitivity of the instrument remains high. As long as these thermal noise triggers do not saturate the data acquisition system, causing deadtime to actual events of interest, this methodology is effective. The thermal noise events so recorded have negligible probability of time alignment to mimic an actual signal. Appendix ?? gives further results demonstrating this conclusion.

b. Trigger Banding In order to provide optimal robustness in the presence of unknown but potentially incapacitating Electro-Magnetic Interference (EMI) backgrounds, a system of non-overlapping frequency bands has been adopted. Typical anthropogenic backgrounds are narrow-band, and while a strong emitter in a given band would likely 
TABLE I: ANITA Electronics Specifications.

\begin{tabular}{|l|c|}
\hline Design Parameter & As-built Value \& Comments \\
\hline \# of RF channels & $80=32$ top, 32 bottom, 8 monitor \\
\hline Sampling rate & $2.6 \mathrm{GSa} / \mathrm{s}$, greater than Nyquist \\
\hline Sample resolution & $\geq 9 \mathrm{bits}=3$ bits noise + dynamic range \\
\hline Samples in window & 260 for a 100 ns window \\
\hline Buffer depth & 4 to allow rapid re-trigger \\
\hline Power/channel & $<10 \mathrm{~W}$ including LNA \& triggering \\
\hline \hline \# of Trigger bands & 4, with roughly equal power per band \\
\hline \# of Trigger channels & 8 per antenna (4 bands x 2 pols.) \\
\hline Trigger threshold & $\leq 2.3 \sigma$ above Gaussian thermal noise \\
\hline Accidental trigger rate & $\leq 5 \mathrm{~Hz}$, gives 'heartbeat' rate \\
\hline Raw event size & $\sim 35 \mathrm{kB}$, uncompressed waveform samples \\
\hline
\end{tabular}

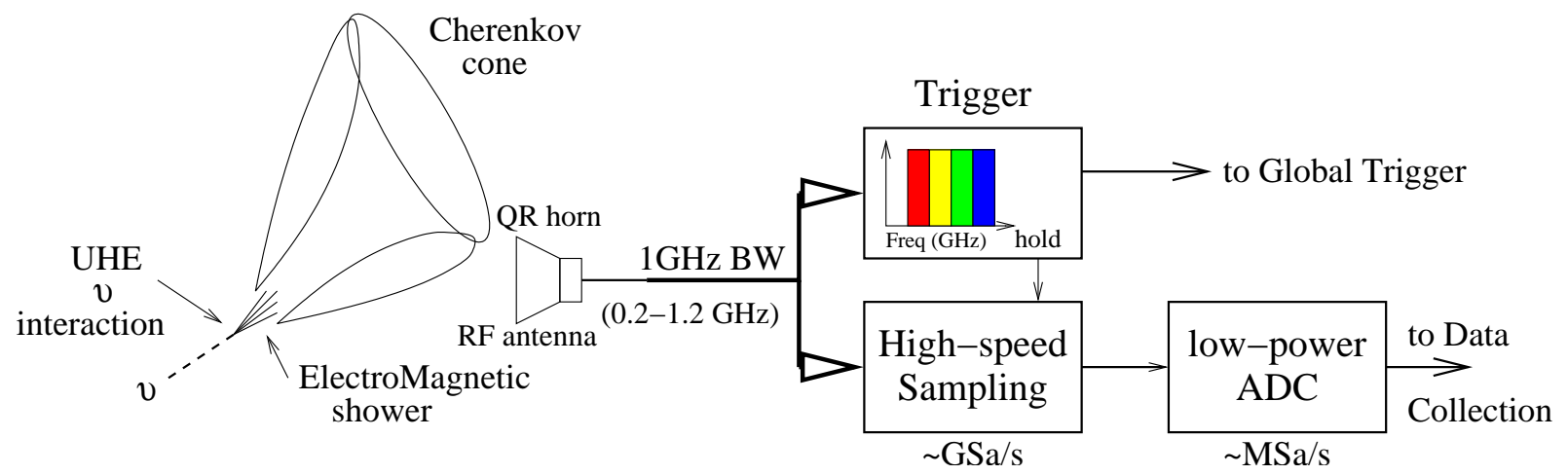

FIG. 8: In order to minimize the power required, signals from the antennas are split into analog sampling and trigger paths. To provide trigger robustness, the full $1 \mathrm{GHz}$ bandwidth is split into 4 separate frequency bands, which serve as separate trigger inputs.

raise the trigger threshold (at constant rate) such that it would be effectively disabled, the other trigger bands could continue to operate at thermal noise levels.

Signals from the vertical and horizontal polarizations of the quad-ridge horn antennas are amplified and conditioned in the RF chain described in the preceding subsection. These RF signals are then split into two paths, a trigger (lower) and digitizer (upper) path as indicated in Fig. 6. The trigger path first passes through the hybrid $90^{\circ}$ combiner which converts the $\mathrm{H} \& \mathrm{~V}$ polarizations to LCP and RCP. The signals then enter a sub-band splitter section where they are divided into frequency bands with centers at $\mathrm{v}_{c}=265,435,650$, and $990 \mathrm{MHz}$ and bandwidths of $\Delta v=130$, $160,270,415 \mathrm{MHz}$, or fractional bandwidths $\Delta \mathrm{v} / \mathrm{v}_{c} \simeq 44 \%$ on average. This partitioning is performed in order to provide rejection power against narrow-bandwidth anthropogenic backgrounds. In contrast, as true Askaryan pulses are temporally compact and coherent, significant RF power is expected across several bands. In addition the thermal noise in each of the bands are statistically independent, and requiring a multi-band coincidence thus permits operation at a lower effective thermal noise floor. This is illustrated schematically in Fig. 9 .

c. Level 1 trigger. The frequency sub-band signals are then led into a square-law-detector section which uses a tunnel-diode, a common technique in radio astronomy. The resulting output unipolar pulses (negative-going in this case, typically $7 \mathrm{~ns}$ FWHM) are filtered and sent to a local-trigger unit (a field-programmable gate array or FPGA) on one of the nine 8-input-channel Sampling Unit for Radio Frequencies (SURF) boards, within the compactPCI ANITA crate, and therefore attached to the host computer and global trigger bus. Within the SURF FPGA, the square-law detector outputs are led through a discriminator section with a programmable threshold. The single-band thresholds are set in a noise-riding mode where they servo on their rate, with a time constant of several seconds, maintaining typical rates of 2.6-2.8 $\mathrm{MHz}$ under pure thermal noise conditions, corresponding approximately to the $2.3 \sigma_{V}\left(\sigma_{V}=V_{r m s}\right.$, the root-mean-square received voltage) level mentioned above. The SURF FPGA also then applies the single-antenna trigger requirement: the eight sub-bands generate a $10 \mathrm{~ns}$ logic level for each signal above threshold, and when any three of these logic gates overlap, a single-antenna trigger is generated. These triggers are denoted Level 1 (L1) triggers, and occur at typical rates of $150 \mathrm{kHz}$ per antenna for thermal-noise conditions. 
SINGLE ANTENNA TRIGGER HEIRARCHY
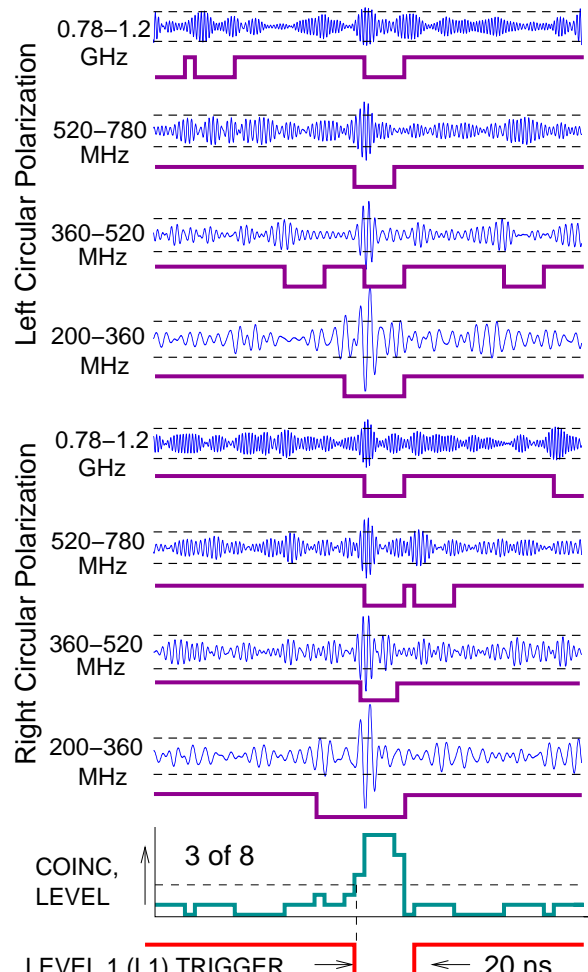

run220 2-of-3 trigger 3 upper+ 3 lower

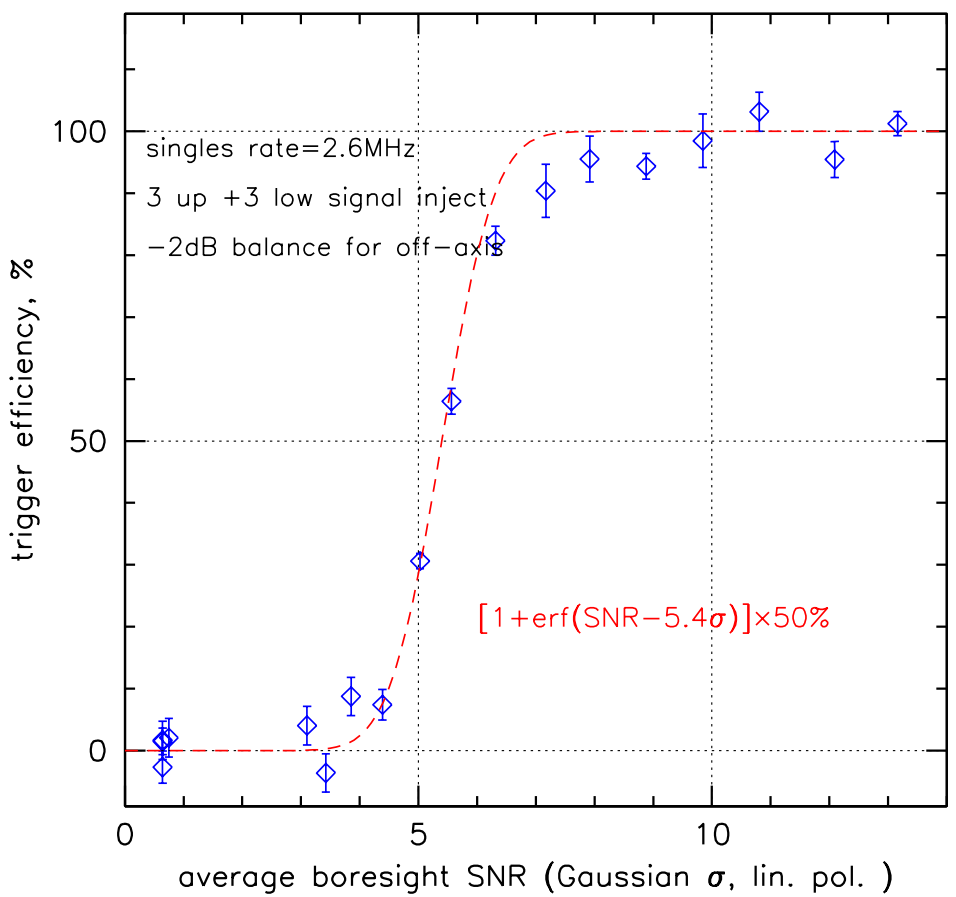

FIG. 9: Left: The baseline single-antenna (L1) triggering is illustrated schematically. In practice the double-sided voltage thresholds shown are implemented by first using a tunnel-diode square-law detector to rectify the band voltage signals into a unipolar pulse. Right: Plot of measured global (L3) trigger efficiency vs. threshold in standard deviations above the RMS thermal noise voltages for ANITA, using a shaped Askaryan-like pulse from a pulse generator under pure thermal noise conditions.

To determine the expected ANITA L1 accidental rate $R_{L 1}$ of $k$-fold coincidences among the $n=8$ sub-band singleantenna channels, consider a trial event, defined by a hit in any one of the $n$ channels, which then triggers a logic transition out of the discriminator to the logic TRUE state for a duration $\tau$. Then consider the probability during this trial that $k-1$ or more ( $k=3$ for ANITA) additional sub-band discriminator logic signals arrive while the first is still in TRUE state, corresponding to a hit above threshold for that channel. The rate of TRUE states per channel is $r$. We do not for now assume $r \tau<<1$.

The probability to observe exactly $k-1$ out of $n-1$ additional channels in the TRUE state after one channel has changed its state is given by the binomial (e.g., the $k$ out of $n$ 'coin toss') probability:

$$
P(k-1: n-1)=\frac{(n-1) !}{(k-1) !(n-k) !} p^{k-1}(1-p)^{n-k} .
$$

The single channel 'coin-toss' probability $p$ is just given by the fractional occupancy of the TRUE state per second per channel: $p=r \tau$.

The probability per trial to observe greater than $k-1$ out of $n-1$ channels is then just the cumulative probability density of the binomial distribution times the observation interval:

$$
P(\geq k-1: n-1)=\sum_{j=k-1}^{n-1} \frac{(n-1) !}{(j) !(n-1-j) !}(r \tau)^{j}[1-r \tau]^{n-j} d t
$$

For $r \tau<<1$ as it often is in practice, this simplifies to

$$
P(\geq k-1: n-1) \simeq \frac{(n-1) !}{(k-1) !(n-k) !}(r \tau)^{k-1}
$$


since only the leading term in the sum contributes significantly and the term $1-r \tau \simeq 1$.

The rate is then determined by multiplying the single-trial probability by the number of ensemble trials per second, which is just equal to the total number of channels times the singles rate per channel. The singles rate per channel is given simply by $r$, and the total singles rate across all channels is $n r$. Thus the total rate in the limit of $r \tau<<1$, is:

$$
R_{L 1}=n r P(\geq k-1: n-1) \simeq n \frac{(n-1) !}{(k-1) !(n-k) !} r^{k} \tau^{k-1}=\frac{(n) !}{(k) !(n-k) !} k r^{k} \tau^{k-1} .
$$

d. Level 2 trigger. When a given SURF module detects an L1 trigger, indicating either a possible signal or (most probably) a thermal noise fluctuation, it reports this immediately to the Trigger Unit for Radio Frequencies (TURF) module, which occupies a portion of the compact-PCI backplane common to all of the SURF modules. The TURF contains another FPGA, which determines whether a level 2 (L2) trigger, which corresponds to two L1 events in any adjacent antennas of either the upper or lower ring, within a $20 \mathrm{~ns}$ window, has occurred. L2 triggers occur at rate of about $2.5 \mathrm{Khz}$ per antenna pair, or about $40 \mathrm{kHz}$ aggregate rate for thermal noise.

e. Level 3 trigger. If a pair of L2s occur in the upper and lower rings within a $30 \mathrm{~ns}$ window and any up-down pair of the antennas share the same azimuthal sector (known as a "phi sector"), a level 3 (L3) global trigger is issued, and the digitization of the event proceeds. These occur at a rate of about $4-5 \mathrm{~Hz}$ for thermal noise. Fig. 9 (right) shows a measurement of the effective threshold for L3 triggers in terms of the peak pulse SNR above thermal noise. Here three upper and three lower antennas were stimulated with a shaped pulse and the L3 rate was measured as a function of the input pulse SNR to estimate the effective global threshold, here in Gaussian standard deviations above the thermal noise RMS voltage. ANITA begins to respond at about $4 \sigma_{V}$, reaches of order $50 \%$ efficiency at $5.4 \sigma_{V}$ and is fully efficient at of order $\sim 7 \sigma_{V}$.

In Appendix B 4, we further analyze the rate of accidentals in terms of their ability to reconstruct coherently to mimic a true signal event, and we find that the chance probability for this is of order 0.003 events for the ANITA flight, presenting a negligible background.
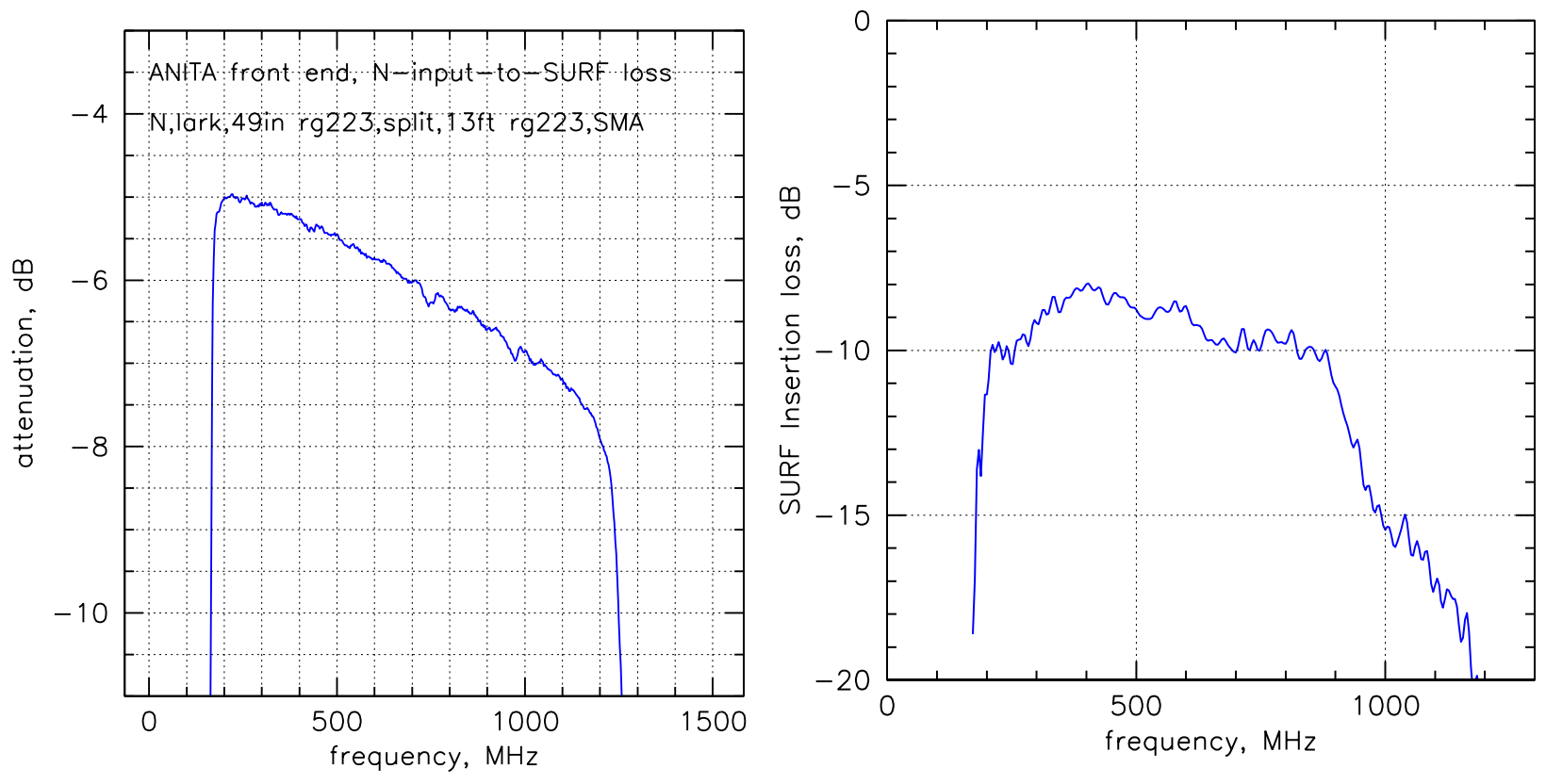

FIG. 10: Left: Detailed view of input signal chain insertion losses, up to the input to the SURF. The losses in the trigger path are slightly lower since the $20 \mathrm{~ns}$ delay cable is omitted. Right:Typical insertion losses for the SURF digitizer inputs used to record the waveforms. The rolloff above about $850 \mathrm{MHz}$ leads to a loss of SNR of the recorded waveforms compared to the actual RF trigger path. 


\section{Digitizer System.}

The upper path in Figure 6 is the digitization path. A low-power, high sampling-speed Switched Capacitor Array (SCA) continuously samples the raw RF inputs over the entire $1 \mathrm{GHz}$ of analog bandwidth defined by the upstream RF conditioning, at a sample rate of 2.6 Gsamples/s. Sampling is halted and the analog waveform samples held for readout upon the fulfillment of a trigger condition. The SCA sampler, which does not actually digitize its stored samples until commanded to do so for a trigger, uses far lower power than traditional high speed continuous-digitizing samplers (such as oscilloscopes). Without the custom development of this technology by G. Varner of the ANITA team, the power budget for ANITA would have grown substantially, from of order $1 \mathrm{~W} / \mathrm{ch}$ to perhaps $10 \mathrm{~W}$ per channel for a commercial digitizer as was used for ANITA-lite [24]. In addition, the continuously sampled data would have added a processing load of order $200 \mathrm{Gbyte} / \mathrm{sec}$ to the trigger system.

Fig. 10 shows measurements of the signal chain and SURF channel insertion loss vs. radio frequency. On the left, the losses up to the input of the SURFs are shown; these are primarily cable and second-stage bandpass filter losses. Similar losses apply to the trigger path as well, though slightly lower since there is no 20 ns delay cable in that path. On the right, the SURF insertion losses are shown, and these are unique to the waveform recording path. The loss above about $850 \mathrm{MHz}$ tend to significantly reduce the intrinsic peak voltages in the most impulsive waveforms compared to what is seen by the analog trigger inputs. These amplitude losses can be corrected to some degree as shown in a later section, but there is still a net loss of SNR in the deconvolved waveforms compared to what is seen by the trigger path.

It is evident from this plot that ANITA's digitizers did not fully achieve the design input bandwidth span of 200-1200 $\mathrm{MHz}$; the last quarter of this band has reduced response compared to the design goal. The main impact of this is not to reduce the trigger sensitivity of the instrument, since the digitizer does not constrain the input bandwidth of the trigger system. Rather, the primary impact is in evaluation of potential neutrino candidates, for which we would like to be able to reconstruct an accurate spectral density for the received radio signal. For the current digitizer system, the reconstructed spectral content above $900 \mathrm{MHz}$ will thus be subject to errors that increase with frequency; however, this frequency region is also the region where ice attenuation is rising quickly [29].

\section{H. Navigation, attitude, \& timing}

a. Absolute Orientation. In order to geometrically reconstruct neutrino events, accurate position, altitude, absolute time, and pointing information are required. To provide such data on an event-by-event basis, a pair of Global Positioning System (GPS) units was used. They provide more than sufficient accuracy to fulfill the science requirements, see Table 20. In addition these units provide the ability to synchronously trigger and read out the system on an absolute timing mark (such as the nearest second), a feature which is essential to the ground-to-flight calibration sequence, where a ground transmitter needs to be globally synchronized to the system during flight, including a propagation delay offset.

ANITA had a mission-critical requirement for accurate payload orientation knowledge, to ensure that the freerotation of the payload would not preclude reconstruction of directions for events at the sub-degree level of accuracy. Such measurements were accomplished with a redundant system of 4 sun-sensors, a magnetometer, and a differential GPS attitude measurement system (Thales Navigation/Magellan ADU5). These systems performed well in flight and met the mission design goals. In calibration done just prior to ANITA's 2006 launch, we measured a total $(\Delta \phi)_{R M S}=$ $0.071^{\circ}$, very close to the limit of the ADU5 sensor specification, and well within our allocated error budget.

The ADU5 is connected to the flight computer with a pair of RS-232 interfaces; one carries the attitude information packets for the housekeeping readout, and the second provides readout when during the UTC second a digital trigger line was set at the ADU5. The second GPS unit, a G12 sensor from Thales, is used to get a second trigger timing piece of information over one serial line and timing information for the flight computer's NTP (Network Time Protocol) internal clock. Position and attitude information is updated every second. Also every second the NTP server gets an update to keep good overall clock time at the computer. The trigger is also connected directly to GPS time: GPS second from the 1 -second readout and fraction of a second from the phototiming data block. Additional pointing information is derived from the sun sensors and a tip-tilt sensor mounted near the experiment's center of mass. They provide a simple crosscheck to the attitude data with very different systematics and also offer a measure of redundancy. 
TABLE II: Navigation, attitude, and timing sensor requirements and provided accuracy.

\begin{tabular}{|l|l|l|l|}
\hline Parameter & Determination method & Required Accuracy & System Accuracy \\
\hline \hline Position/Altitude & Ordinary GPS & $10 \mathrm{~m}$ horiz./20m vert. & 5m Horizontal/10m Vertical \\
UTC & GPS Phototiming pulse & $20 \mathrm{~ns}$ & $<1$ ns \\
Pointing & Short-baseline differential GPS & $0.3^{\circ}$ rotation $/ 0.3^{\circ}$ tip & $<0.07^{\circ} /<0.14^{\circ}$ \\
Pointing Sun-sensor & Tip-tilt sensor & $0.3^{\circ}$ rotation $/ 0.3^{\circ}$ tip & $1^{\circ} / 1^{\circ}$ \\
\hline
\end{tabular}

\section{FLIGHT SOFTWARE \& DATA ACQUISITION}

The ANITA-I flight computer was a standard c-PCI single board computer, based on the Intel Mobile Pentium III CPU. The operating system was Red Hat 9 Linux, which was selected due to driver availability.

The design philosophy behind the ANITA flight software was to create, as far as possible, autonomous software processes. Inter-process data transfer consists of FIFO queues of data files and links on the system ramdisk, these queues are managed using standard Linux filesystem calls. Process control is achieved using configuration files and a small set of POSIX signals (corresponding to: re-read config files, restart, stop, and kill). A schematic overview of the flight software processes is shown in Figure 11.

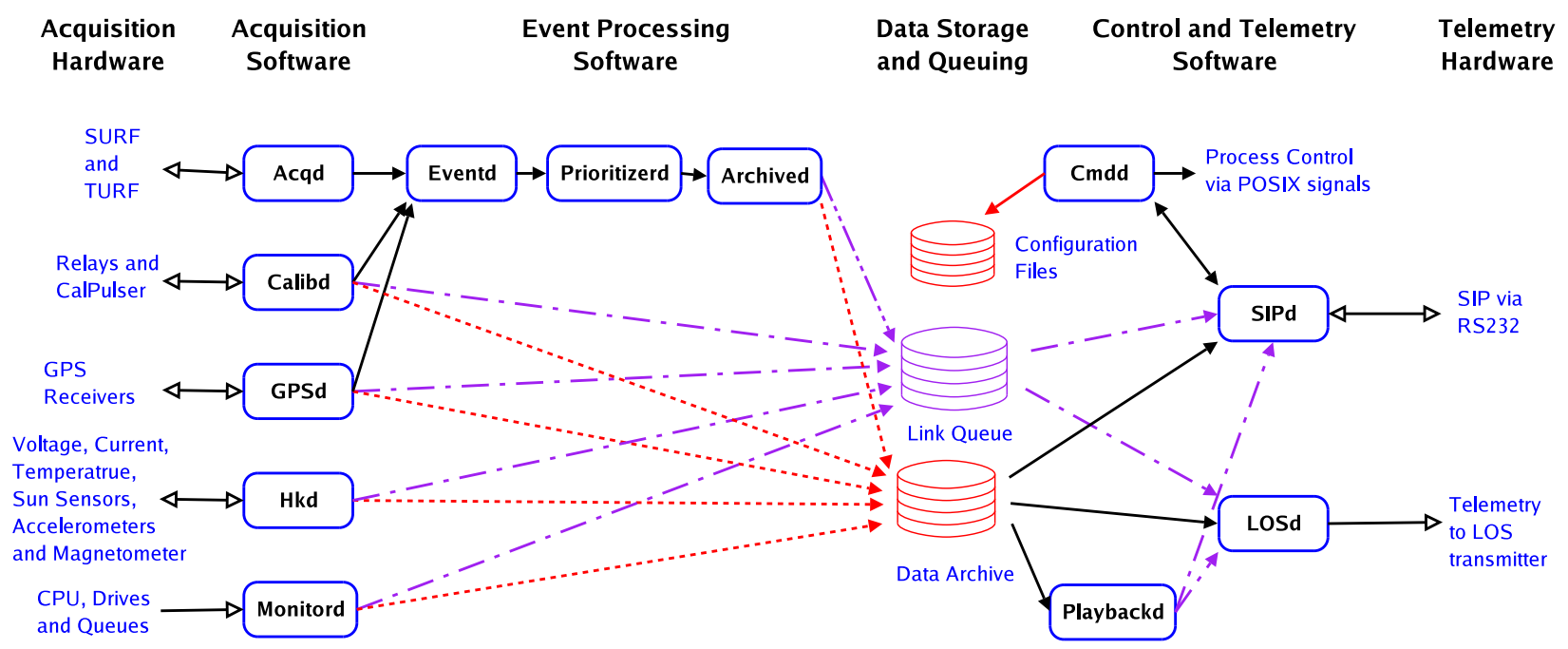

FIG. 11: A schematic overview of the flight software processes. The open arrows show software interaction with hardware components, and closed arrows indicate data transfer between processes. The telemetry data flow across the ramdisk is indicated by the dot-dashed (purple) line, and permanent data storage to the on-board drives is shown by the the dotted (red) lines.

The flight software processes break down into three main areas, which will be discussed in following sections. These areas are:

1. Data acquisition - processes which control specific hardware and through which all data from that hardware is obtained.

2. Event processing - processes which augment or analyze the waveform data in the on-line environment.

3. Control and telemetry - processes which control the telemetry hardware and those which are responsible for process control.

\section{A. Data Acquisition}

The bulk of the data, around 98\%, acquired during the flight is in the form of digitized waveform data from the SURF boards. The remaining $2 \%$ consists of auxiliary information necessary to process and interpret the waveform 
data (payload position, trigger thresholds, etc.) and data that is used to monitor the health of the instrument during flight (temperatures, voltages, disk spaces, etc.).

\section{Waveform Data}

The process that is responsible for the digitization and triggering hardware, the SURF and TURF, is Acqd (the Acquisition Daemon). The Acqd process has four main tasks:

- Acquiring waveform data from the SURFs

- Acquiring trigger and timing data from the TURF (via TURFIO).

- Acquiring housekeeping data from the SURF (scaler rates, read back thresholds, and RF power).

- Setting the thresholds and trigger band masks to control the trigger, dynamically adjusting the thresholds to maintain a constant rate.

Once the TURF has triggered an event and the SURFs' have finished digitizing, the event data is available for transfer across the c-PCI backplane to the flight computer. The flight computer polls the SURFs to check when an event has finished digitization and the data is ready to be transferred across the c-PCI backplane.

An event consists of 260 16-bit waveform data words per channel, there are 9 channels per SURF and 9 SURFs in the c-PCI crate. A complete raw event is approximately $41 \mathrm{~KB}$. To achieve better compression, see Section IV B 2 , the raw waveform data is pedestal subtracted before being written to the queue for event processing.

\section{Trigger Control}

In addition to acquiring the waveform and trigger data, Acqd is also responsible for setting the thresholds and trigger band masks that control the trigger. There are three handles through which Acqd can control the trigger:

- The single channel trigger thresholds (256 channels).

- The trigger band masks (8 channels per antenna).

- The antenna trigger mask (32 antennas in total).

The default mode of operation is to have all of the masks off, such that every trigger band and every antenna can participate in the trigger. In the thermal regime, i.e. away from anthropogenic RF noise sources such as camps and bases, the trigger control operates by dynamically adjusting the single channel thresholds to ensure that each trigger channel triggers at the same rate (typically $2-3 \mathrm{MHz}$ ). The dynamic adjusting of the thresholds is necessary as even away from man-made noise sources the RF power in view varies with the temperature of the antenna and its field of view, i.e with the position of the sun and galactic center with respect to the antenna. The thresholds are varied using a simple PID (proportional integral differential) servo loop that was tuned in the laboratory using RFCMs with terminated inputs.

During times when the balloon is in view of large noise sources, such as McMurdo station, a different triggering regime is necessary to avoid swamping the downstream processes with an unmanageable event rate. To allow for this all of the trigger control options are commandable from the ground, see Section IV C2 for more details on commanding. Using these commands some of the available options for controlling the trigger rate are:

- Adjust the global desired single trigger channel rate.

- Adjust individual single channel rates independently.

- Remove individual trigger channels (i.e. frequency bands) from the antenna level (L1) trigger.

- Remove individual antennas from the L2 and L3 triggers. 


\section{Housekeeping Data}

In addition to the waveform data, housekeeping data is also continuously captured, both for use in event analysis and also for monitoring the health of the instrument during flight. Table III is a summary of the various types of housekeeping data acquired by the flight software processes.

\begin{tabular}{|c|l|c|}
\hline Process & Housekeeping Data & Rate \\
\hline Acqd & Trigger rates and average RF power & up to $5 \mathrm{~Hz}$ \\
Calibd & Relay status & $0.03 \mathrm{~Hz}$ \\
GPSd & GPS position, velocity, attitude, satellites, etc. & up to $5 \mathrm{~Hz}$ \\
Hkd & Voltages, currents, temperatures, pressures, etc. & up to $5 \mathrm{~Hz}$ \\
Monitord & CPU and disk drive status & $0.03 \mathrm{~Hz}$ \\
\hline
\end{tabular}

TABLE III: The types of housekeeping data acquired by flight software processes.

\section{B. On-line Event Processing}

At altitude the bandwidth for downloading data from the payload to the ground systems is very limited, see Section IV C 1. In order to maximize the usage of this limited resource the events are processed on-line to determine the event priority and they are compressed and split into a suitable format for telemetry.

\section{Prioritization}

The Prioritizerd daemon is responsible for determining the priority of an event. This priority is used to determine the likelihood of a given event being telemetered to the ground during flight. The prioritizer looks at a number of event characteristics to determine priority. The hierarchical priority determination is described below:

- Priority 9 - If too many waveforms (configurable) have a peak in the FFT spectrum (configurable), the event is given this low priority, to veto events from strong narrow band noise sources.

- Priority 8 - If two many channels peak simultaneously, determined via matched filter cross-correlation, the event is assumed to be generated on-payload and is rejected.

- Priority 7 - Compares the RMS of the beginning and end of waveforms to veto non-impulsive events.

- Priority 6 - This is the default priority. Thermal noise events will be assigned this priority if they are not demoted for one of the above reasons, or promoted for one of the below reasons.

- Priority 5 - An event is promoted to priority five if it passes the test of $\mathrm{N}$ of $\mathrm{M}$ (configurable) neighboring antennas peaking within a time window (configurable). Events must satisfy this condition to be onsidered for promotion to priorities 1-4.

- Priority 4 - A cross-correlation is performed with boxcar smoothing, and there are peaks in 2-of-2 antennas in one ring and 1-of-2 in the other.

- Priority 3 - A cross-correlation is performed with boxcar smoothing, and there are peaks in 2-of-3 antennas in both rings.

- Priority 2 - A cross-correlation is performed with boxcar smoothing, and there are peaks in 2-of-2 antennas in both rings.

- Priority 1 - A cross-correlation is performed with boxcar smoothing, and there are peaks in 3-of-3 antennas in both rings. 


\section{Compression}

Several encoding methods were investigated for the telemetry of waveform data. Of the methods investigated, the optimum method was determined to be a combination of binary and Fibonacci encoding. The steps involved in the compression are described below:

- The waveform is pedestal subtracted and zero-meaned.

- The data is truncated to 11-bits (the lowest bit in the data is shorted to the next to lowest)

- A binary bit size is determined based upon the smallest power of two above the three standard deviation point of the waveform.

- All samples that lie within the range of values that can be encoded with a binary number of this dimension are encoded as their binary value, those outside are assigned the maximal value.

- The difference between the maximal value and the actual sample value are then encoded using Fibonacci coding in an overflow array. Fibonacci coding is useful for this purpose as it efficiently encodes large numbers, and it has some built in immunity to data corruption as each encoded number ends with 11.

- The full encoded waveform then consists of $260 n$-bit binary numbers followed by $M$ Fibonacci encoded overflow values.

Figure 12 shows the performance of the binary/Fibonacci encoding method in comparison with the other methods considered. This method was chosen as it proved to give the best compression of the loss-less methods considered.

\section{RF Channel Comparison}

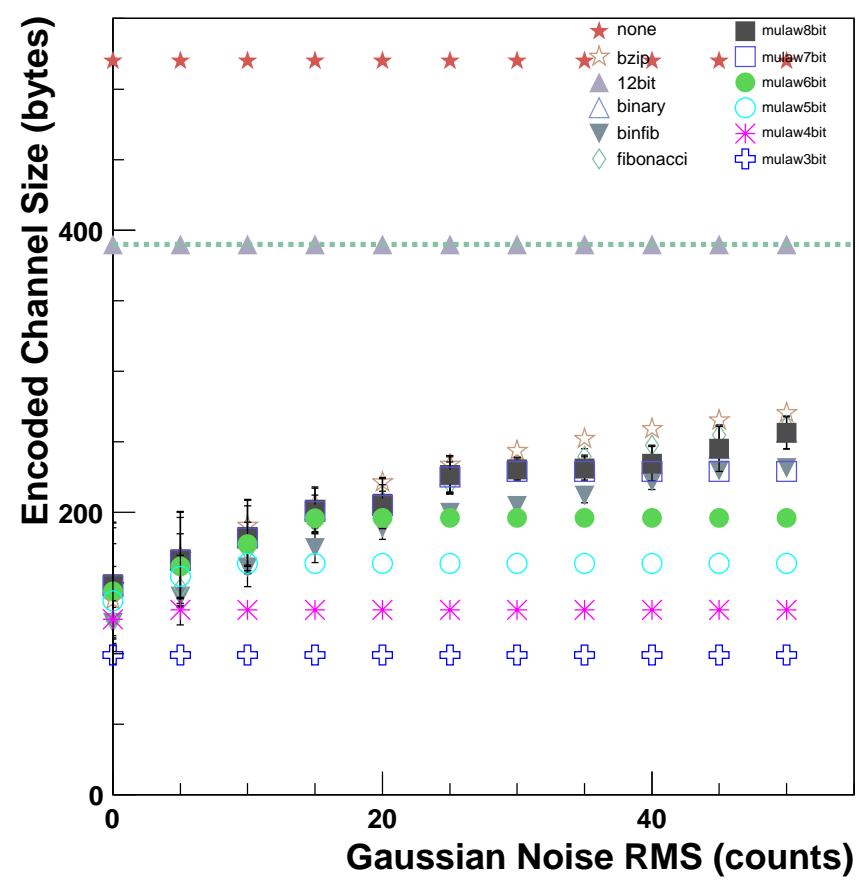

FIG. 12: A comparison of the performance of the different loss-less and lossy compression methods tested on ANITA waveform data with adjustable Gaussian noise levels. The binary/Fibonacci method detailed in the text proved to be the most effective method for encoded the telemetry data. The mu-law methods were the only lossy methods that were considered. 


\section{Control and Telemetry}

One critical aspect of the flight software for a balloon experiment is the control telemetry software. This software represents the only link between the experiment and the scientists on the ground. As such the software needs to be both very robust, to withstand a long flight away from human interaction, and also flexible enough to cope with unexpected failures during the flight.

\section{Data Downlink}

During the flight it is critical to telemeter enough information that the scientists on the ground can determine whether the instrument is operating, and acquiring sensible data. All of this information needs to be pushed through one of two narrow (bandwidth limited) pipes to the ground. When close to the launch site the data is sent over a Line of Sight (LOS) transmitter with a maximum bit rate of 300kbps. Once over the horizon the only data links available are satellite links: i) a continuous $6 \mathrm{kbps}$ using the TDRSS satellite network; and ii) a maximum of 248 bytes every 30 seconds using the IRIDIUM network. The ODIUM link is only used to monitor payload health during times when the other, higher bandwidth, links are unavailable (due to satellite visibility and other issues).

There are several data streams that are telemetered, these are summarized in Table IV. Clearly, only a tiny fraction of the total data written to disk is able to be telemetered to the ground using the satellite links.

\begin{tabular}{|c|c|c|c|}
\hline Data Type & Size (bytes) & TDRSS (packets/min) & LOS (packets/min) \\
\hline Header & 74 & 150 & 300 \\
Waveform & 14,000 & 2 & 120 \\
GPS & 88 & 6 & 300 \\
Housekeeping & 150 & 6 & 60 \\
RF Scalers & 1380 & 1 & 30 \\
CPU Monitor & 64 & 1 & 1 \\
\hline
\end{tabular}

TABLE IV: A summary of the data types, sizes and telemetry rates over the LOS and TDRSS downlinks.

\begin{tabular}{|c|c|c|}
\hline Command Name & Description & Number \\
\hline Send Config Files & Telemeter config file & $\sim 300$ \\
Set PID Goal & Adjust the single band trigger rate & $\sim 250$ \\
Send Log Files & Telemeter log file & $\sim 250$ \\
Kill Process & Tries to kill the selected process & $\sim 150$ \\
Restart Process & Tries to restart the selected process & $\sim 100$ \\
Set Band Mask & In/Exclude frequency bands & $\sim 100$ \\
TURN RFCM On/Off & Turn on/off the power to the amplifiers & $\sim 45$ \\
Set Antenna Mask & In/Exclude antennas from the trigger & $\sim 20$ \\
GPS Trigger Flag & Enable/Disable GPS triggered events & $\sim 20$ \\
Start New Run & Starts a new run & $\sim 20$ \\
Set Channel Scale & Change the goal rate of an individual band & $\sim 15$ \\
\hline
\end{tabular}

TABLE V: The most common commands used during the ANITA-I flight, with a brief description of the intended command outcome, and an indication of the number of times the command was used during the ANITA-I flight.

\section{Commanding}

During the flight it is possible to send commands from the ground to the payload over one of the three available links: line of sight, TDRSS and IRIDIUM. Each command contains up to 20 bytes of information. The most common commands used during the ANITA-I flight are shown in Table $\mathrm{V}$. 


\section{MONTE CARLO SIMULATION SENSITIVITY ESTIMATES}

ANITA's primary goal is the detection of the cosmogenic neutrino flux. All first-order modeling of such fluxes assume they are quasi-isotropic; that is to say, although they originate from sources of small angular extent and retain their directional information throughout their propagation trajectory to earth, the sources are presumed to be distributed in a spatially uniform manner as projected on the sky and viewed from Earth. Thus to a good approximation we model the incoming neutrino flux as sampling a parent distribution that is uniform across the spherical sky.

Because of the complexity of the neutrino event acceptance for the target volume of ice that ANITA's methodology employs, a complete analytic estimate of the neutrino detection sensitivity is very difficult, and we have relied on detailed Monte Carlo simulation methods to perform the appropriate multi-dimensional integration which determines the acceptance as a function of neutrino energy.

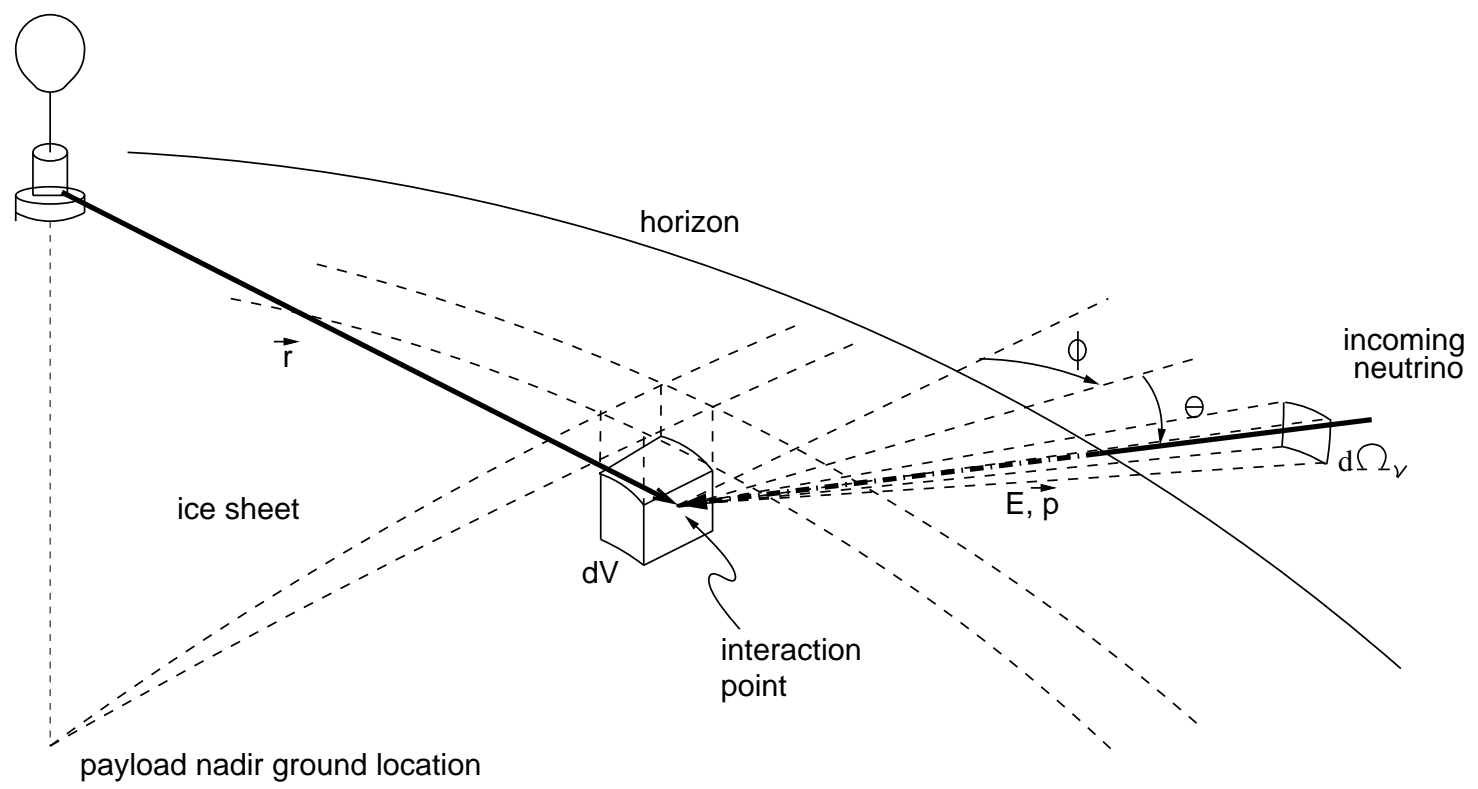

FIG. 13: Schematic view of the geometry for a neutrino interaction in the field-of-view of ANITA.

One may visualize the acceptance by first considering a volume element located somewhere within the horizon of the ANITA payload at some instant of time, at a depth within several electric field attenuation lengths of the ice surface. In the absence of the surrounding ice and earth below this volume element, the neutrino flux passing through such a volume is isotropic. However, the attenuation due to the earth and surrounding ice modifies the flux and resulting acceptance, so that, at EeV energies where neutrino attenuation lengths are several hundred km water equivalent for the standard model cross sections, most of the neutrino acceptance below the horizon is lost. A schematic view of the geometry is shown in Fig. 13. The volume element $d V$, and position $\vec{r}$ with respect to the payload's instantaneous location, encloses an interaction location which is subject to a neutrino flux over solid angle element $d \Omega_{v}$, at offset angles $\phi, \theta$ with respect to the radial azimuth from the payload and local horizontal directions. Note that the payload angular acceptance $d \Omega$ is related to $d \Omega_{v}$ by convolution with the detectability of neutrinos at every location within the field of view. The geometry of the Cherenkov cone and refraction effects then complicate the convolution even further, and Monte Carlo integration becomes an attractive way to deal with this complexity.

We may still define an average volumetric acceptance $\langle\mathcal{V} \Omega\rangle$, in $\mathrm{km}^{3}$-water-equivalent steradians $\left(\mathrm{km}^{3} \mathrm{we} \mathrm{sr}\right)$, as the physical target density-times-volume $\rho V$ of the detector multiplied by the weighted solid angle $\Omega_{v}$ over which initially isotropic neutrino fluxes produce interactions within the detector, and then multiplied again by the fraction $N_{\text {det }} / N_{\text {int }}$ of such interactions that are detected. For any given volume element of the target, the latter term will depend on the convolution of the emission solid angle for detectable radio signals with the arrival solid angle of the neutrinos. Although the symbol $\mathcal{V} \Omega$ appears to imply that the two parts of the acceptance (volume and solid angle) can be factored, this is generally not true in practice since they tend to be strongly convolved with one another as a function of energy. That is to say, any given volume element may provide events detectable over a certain solid angle centered on some portion of the sky, but a different volume element will in general have both a different net solid angle for detection and also a different angular region over which those detections occur, all as a function of energy. However, 
we can define an average acceptance solid angle $\langle\Omega\rangle$ and this factor is a useful quantity in this calculation.

The differential number of neutrino interactions per unit time, per unit neutrino arrival solid angle, per unit volume element in the detector target, can be written as:

$$
\frac{d^{3} N_{i n t}}{d t d \Omega_{v} d V}=\int_{E_{t h r}}^{\infty} d E_{\mathrm{v}} F_{\mathrm{v}}\left(E_{\mathrm{v}}\right) \sigma_{v N, e}\left(E_{\mathrm{v}}\right) \rho(\vec{r}) N_{A} P_{s u r v}\left(E_{\mathrm{v}}, \vec{r}, \theta_{\mathrm{v}}, \phi_{\mathrm{v}}\right)
$$

where $E_{t h r}$ is the threshold energy for the detector, $\sigma_{v N, e}$ the neutrino total cross section on nucleons (or electrons, for $v_{e}+e$ scattering), $F_{\mathrm{v}}\left(E_{\mathrm{v}}\right)$ is the neutrino flux as a function of energy, $\rho(\vec{r})$ is the ice density at interaction position $\vec{r}$, $N_{A}$ is Avogadro's number, and $P_{\text {surv }}\left(E_{v}, \vec{r}, \vec{p}_{v}\right)$ is the survival probability for a neutrino of energy and 3-momentum $E_{v}, \vec{p}_{v}$ to arrive at position $\vec{r}$, which is the location of the volume element under consideration. This probability can be further written:

$$
P_{\text {surv }}\left(E_{\mathrm{v}}, \vec{r}, \theta_{v}, \phi_{\mathrm{v}}\right)=e^{-L_{\text {int }}\left(E_{\mathrm{v}}\right) X\left(\vec{r}, \theta_{\mathrm{v}}, \phi_{\mathrm{v}}\right)}
$$

with the neutrino interaction length $L_{i n t}=\left(\sigma_{v N, e} N_{A}\right)^{-1}$, and the function $X\left(\vec{r}, \theta_{v}, \phi_{v}\right)$ the total column density along direction $\theta_{v}, \phi_{v}$ from point $\vec{r}$ within the detector. The function $X$ thus contains the earth-attenuation dependence.

The number of neutrino interactions is thus

$$
N_{i n t}=\int_{0}^{T} d t \int_{0}^{4 \pi} d \Omega_{\mathrm{v}} \int_{0}^{V} d V \frac{d^{3} N_{i n t}}{d t d \Omega_{V} d V}
$$

To determine the average acceptance solid angle $\langle\Omega\rangle$, imagine the detector target volume completely isolated from any exterior target matter of the earth, bathed in an isotropic flux of neutrinos from $4 \pi$ solid angle, with the assumption that the interaction length is large enough compared to the target thickness that we may neglect self-attenuation within the target. The number of interactions for this idealized case is

$$
N_{i n t, 0}=\int_{0}^{T} d t \int_{4 \pi} d \Omega_{v} \int_{V} d V \int_{E_{t h r}}^{\infty} d E_{v} F_{V}\left(E_{\mathrm{v}}\right) \sigma_{v N, e}\left(E_{\mathrm{v}}\right) \rho(\vec{r}) N_{A}
$$

or the same integral as equation 6 except that the survival probability $P_{\text {surv }}=1$ for all arrival directions. With this prescription, the average acceptance solid angle is given by

$$
\langle\Omega\rangle=4 \pi \frac{N_{i n t}}{N_{\text {int }, 0}}
$$

The corresponding number of detections depends both on the interactions and the probability $P_{\text {det }}$ of detection of the resulting shower. To first order, deep inelastic neutrino charged-current interactions lead to an immediate local hadronic shower and a single charged lepton which escapes the vertex and can subsequently interact. For electron neutrinos at $10^{18-20} \mathrm{eV}$, this lepton interaction usually takes place rapidly, and produces an immediate secondary electromagnetic shower, which will be elongated due to Landau-Pomeranchuk-Migdal (LPM) suppression of the bremsstrahlung and pair-production cross sections. For other neutrino flavors, the secondary lepton will propagate long distances through the medium but can produce detectable secondary showers through electromagnetic (bremsstrahlung or pair production) or photo-hadronic processes. Since the average Bjorken inelasticity $\left\langle y\left(E_{v}\right)\right\rangle \simeq 0.22$ at these energies, the secondary lepton in these charged current interactions leaves with most of the energy on average, so a secondary shower with any appreciable fraction of the lepton's energy may exceed the hadronic vertex in shower energy. Accounting for this, the number of detections can be written

$$
N_{d e t}=\int_{0}^{T} d t \int_{4 \pi} d \Omega_{v} \int_{V} d V \frac{d^{3} N_{i n t}}{d t d \Omega_{v} d V}\left[P_{d e t, h}\left(y E_{v}, \vec{r}_{h}, \theta_{v}, \phi_{v}\right)+\alpha_{c c} P_{d e t, \ell}\left((1-y) E_{v}, \vec{r}_{\ell}, \theta_{v}, \phi_{v}\right)\right]
$$

where $P_{\text {det }, h}\left(y E_{v}, \vec{r}_{h}, \theta_{v}, \phi_{v}\right)$ is the detection probability for the hadronic showers as a function of shower energy $y E_{v}$, shower centroid position $\vec{r}_{h}$, and shower momentum angles $\theta_{v}, \phi_{v}$. The corresponding detection probability for subsequent lepton showers is written as

$$
\alpha_{c c} P_{d e t, \ell}\left((1-y) E_{v}, \vec{r}_{\ell}, \theta_{v}, \phi_{v}\right)
$$

where $\vec{r}_{\ell}$ is the centroid of the leptonic shower and $\alpha_{c c}=1,0$ for charged- or neutral-current interactions respectively (the latter have zero detection probability since the outgoing lepton is a neutrino; for simplicity in this present treatment 
we do not include subsequent possible neutrino interactions from neutral-current events within the detector and their contribution is small in any case).

Once the two integrals given by $N_{\text {int }}$ and $N_{\text {det }}$ are determined, the volumetric acceptance is given by

$$
\langle\mathcal{V} \Omega\rangle=\frac{N_{\text {det }}}{N_{\text {int }}} V_{0} \Omega=4 \pi V_{0} \frac{N_{\text {det }}}{N_{\text {int }, 0}}
$$

where $V_{0}$ is the total physical volume over which the trial neutrino flux arrives.

\section{A. Monte Carlo Implementation}

We have developed two largely independent simulation codes, one at the University of Hawaii, hereafter denoted the Hawaii code, and a second originally developed at UCLA, but now maintained at University College London, hereafter denoted the UCL code. Although some empirical parameterizations (for example, ice refractive index vs. depth) may use common code or source data, the methodologies of the two codes are entirely independent.

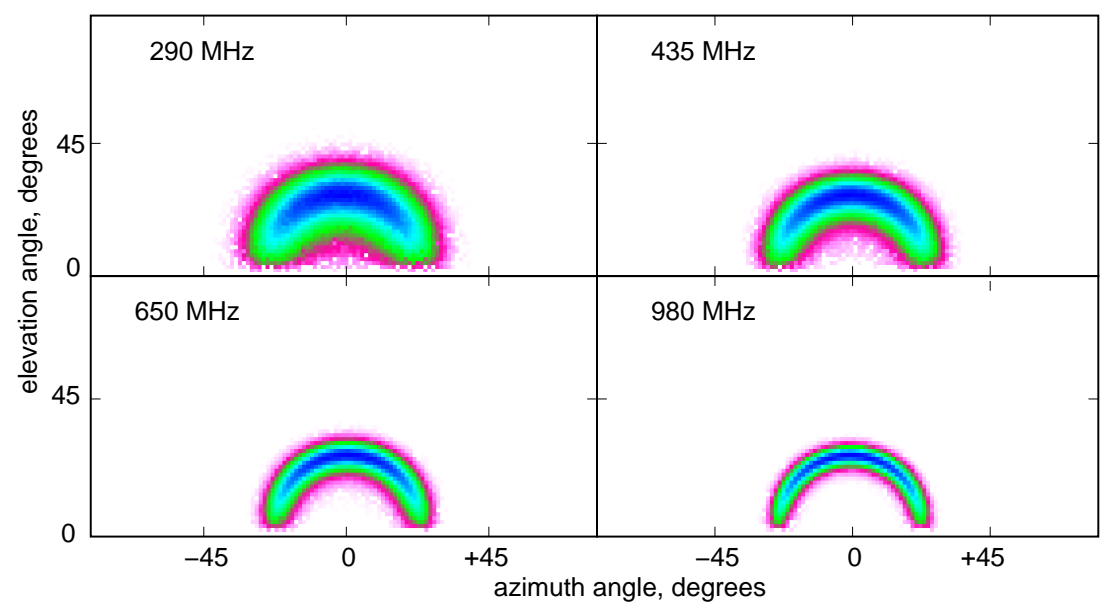

FIG. 14: Simulated false-color images of relative E-field strength of the emerging Cherenkov cone as projected onto the sky due to a neutrino event at energy of order $3 \times 10^{18} \mathrm{eV}$, with an upcoming angle of several degrees relative to the local horizon. The different panes show the response at different radio frequencies as indicated. The color scale is normalized to the peak (blue) in each pane; in fact the higher frequencies peak at higher field strengths.

Both the Hawaii and UCL simulations of the ANITA experiment estimate the experiment's sensitivity from a sample of simulated ultra-high energy neutrino interactions in Antarctic ice. The programs use variations of ray tracing methods to propagate the signal from the interaction to the payload antennas, and then through the three-level ANITA instrument trigger. The neutrino events can be drawn from a parent sample that match any given source spectrum, or, for more general results, the acceptance can be estimated as a function of energy by stepping through a series of monoenergetic neutrino fluxes to map out the energy dependence. This latter approach then allows for estimates of the acceptance which can be convolved with any input neutrino flux.

The two simulations also differ in the areas in which they focus their highest fidelity. The Hawaii code attempts to model the entire pattern of the sky brightness of radio emission produced by a neutrino interaction, creating a library of these events which is later sampled for different relative sky positions of the payload with respect to the events. Fig. 14 (left) shows one example of this type of modeling, in this case for a smooth ice surface. This skybrightness modeling includes also a first-order model for the wavelength-scale surface roughness of the ice. The method uses a facet-model of the surface, and probes it with individual ray traces that are distributed in their number density according to the Cherenkov cone intensity (see Fig. 33 included in an Appendix). This surface ray-sampling thus gives a first-order approximation that has been found to give reasonable fidelity for such effects as enhanced transmission coefficients beyond the total-internal-reflectance angle, for example. Additional detail regarding surface roughness effects are included in an Appendix. 

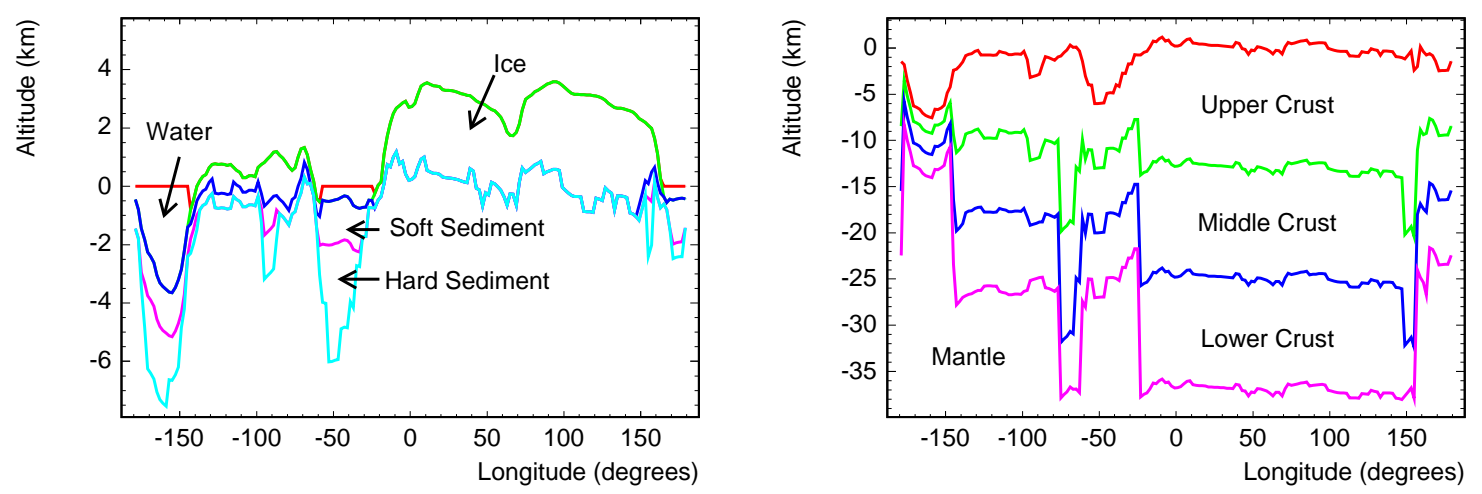

FIG. 15: Left: Altitude of the upper four layers given in Crust 2.0 along the the $75^{\circ} \mathrm{S}$ latitude line. The horizontal axis is degrees in longitude. Right: Altitude of the lower three layers given in Crust 2.0 along the the $75^{\circ} \mathrm{S}$ latitude line.

The Hawaii code does not attempt detailed modeling of the physical topography of Antarctica but instead uses a standard ice volume with homogeneous characteristics, and estimates of the actual ANITA flight acceptance use a weighted-average based on the piecewise geography of the flight path.

The UCL simulation, in contrast, does include a detailed modeling of the Antarctic ice sheets and subcontinent based on the CSEDI Crust 2.0 model of the Earth's interior, and the BEDMAP model for Antarctic ice thicknesses. Figure 15 shows the elevations of the seven crustal layers included in Crust 2.0; the BEDMAP database provides similar resolution. This approach provides higher fidelity estimates of the instantaneous ice volume below the payload for any given time during the flight. This approach toward event modeling, with its limited sampling of ray propagation, does not easily lend itself to treatment of surface roughness however, but is optimized for speed, using a principal-ray approximation for the neutrino interaction-to-payload radiation path. Thus each event is observed along a single ray refracted through the surface from the cascade location.

Despite their distinct differences in approach, the two methods have converged to good agreement in the total neutrino aperture, when compared for a given standard ice volume. Details of the two methods are described in two appendices.

In Fig. 16 we show the results of the two estimates of total effective volumetric acceptance (volume times acceptance solid angle for an isotropic source such as the cosmogenic neutrinos) as estimated by the UH and UCL simulations. The values may be thought of as the total water-equivalent volume of material that has 1 steradian of acceptance to a monoenergetic flux of neutrinos at each plotted energy. It is evident that there is reasonably good agreement for ANITA's acceptance, with values that differ by of order a factor of 2 at most.

In practice the solid angle of acceptance for any volume element of the ice sheet surveyed by ANITA is in fact very small, and the acceptance is also quite directional, covering only a relatively small band of sky at any given moment. The actual sky coverage will be shown in the next section.

\section{PRE-FLIGHT CALIBRATION}

In June of 2006 prior to the ANITA flight, the complete ANITA payload and flight system was deployed in End Station A at the Stanford Linear Accelerator Center (SLAC) about $13 \mathrm{~m}$ downstream of a prepared target of 7.5 metric tons of pure carving-grade ice maintained at $-20 \mathrm{C}$ in a refrigerated container. Using the $28 \mathrm{GeV}$ electron bunches produced by SLAC, electromagnetic showers were created in the ice target, resulting in Askaryan impulses that were detected by the payload with its complete antenna array, trigger, and data acquisition system. This beam test experiment thus provided an end-to-end calibration of the flight system, and yielded on- and off-axis antenna response functions for ANITA as well as a separate verification of the details of the Askaryan effect theory in ice as the dielectric medium.

A complete report of the results for the Askaryan effect in ice has been presented in a separate report [21], which we denote as paper I, and we do not reproduce that material here. However, antenna response details were not reported in paper I, so we provide those here since they are relevant to the ANITA-1 flight. Fig. 17(top) shows an antenna impulse 


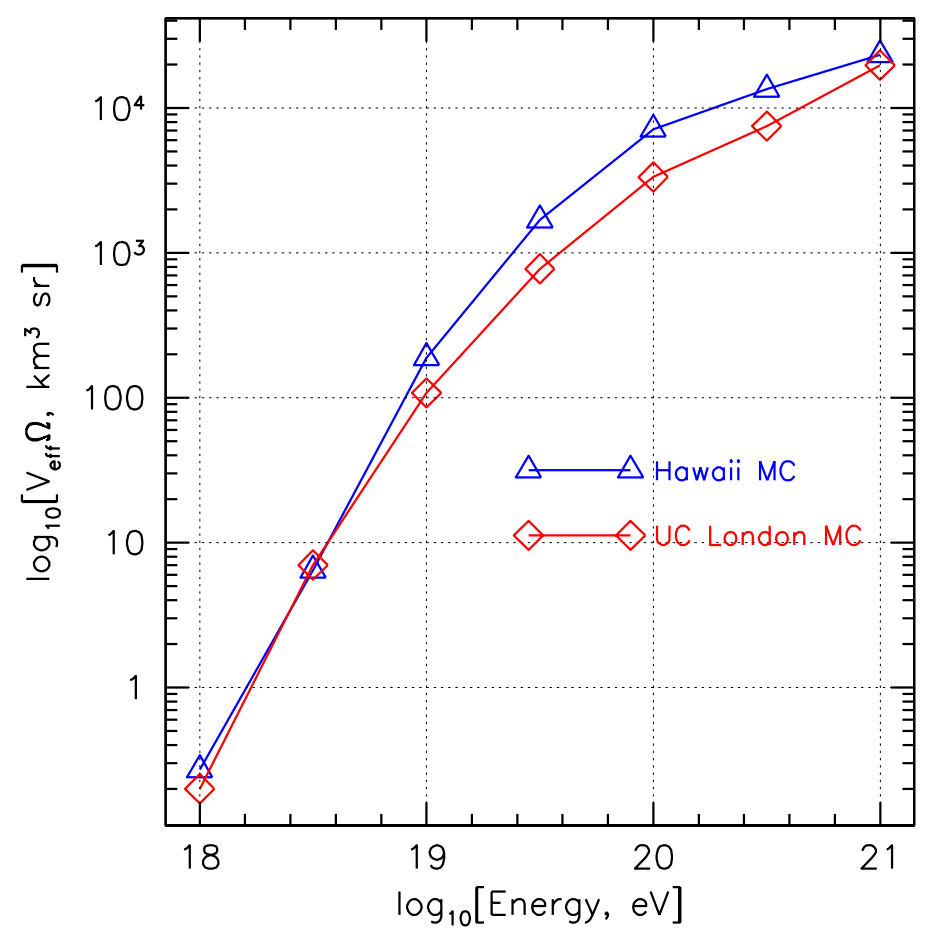

FIG. 16: Curves of simulated acceptance vs. energy for the two independent ANITA Monte Carlos.

response function, and (bottom) the same set of response functions for the actual Askaryan signals recorded during the SLAC beamtest. The dashed and solid lines show both raw and partially deconvolved to remove the lowest-frequency component, which gives the long tail of $\sim 200 \mathrm{MHz}$ ringing that is seen in the plots. This tail is due to the rapid rise in group-delay as one approaches the low-frequency cutoff of the antenna, and although it can be useful as a kind of "fingerprint" of the antenna response in the presence of noise, it does not contribute much power to the actual trigger. The solid lines show the response function with the low-frequency component removed by wavelet deconvolution, showing the dominant power components. These data may be compared to the laboratory measurements in Fig. 5 above.

\section{IN-FLIGHT PERFORMANCE}

ANITA's primary operational mode during the flight was to maintain the highest possible sensitivity to radio impulses with nanosecond-scale durations, but the trigger was designed to be as loose as possible to also record many different forms of impulsive interference. The single-band thresholds were allowed to vary relative to the instantaneous radio noise. The system that accomplished this noise-riding behavior utilized a Proportional-Integral-Differential (PID) servo-loop with a typical response time of several-seconds based on several $\mathrm{Hz}$ sampling of the individual frequency band singles rates.

\section{A. Antenna temperature}

The power received by the antennas in response to the ambient RF environment directly determines the limiting sensitivity of the instrument. This received system power $P_{S Y S}$, referenced to the input of the low-noise amplifier (LNA) is often expressed in terms of the effective temperature, via the Nyquist relation [48], which can be expressed in simple terms as:

$$
P_{S Y S}=k T_{S Y S}=k\left(T_{A N T}+T_{R C V R}\right)
$$



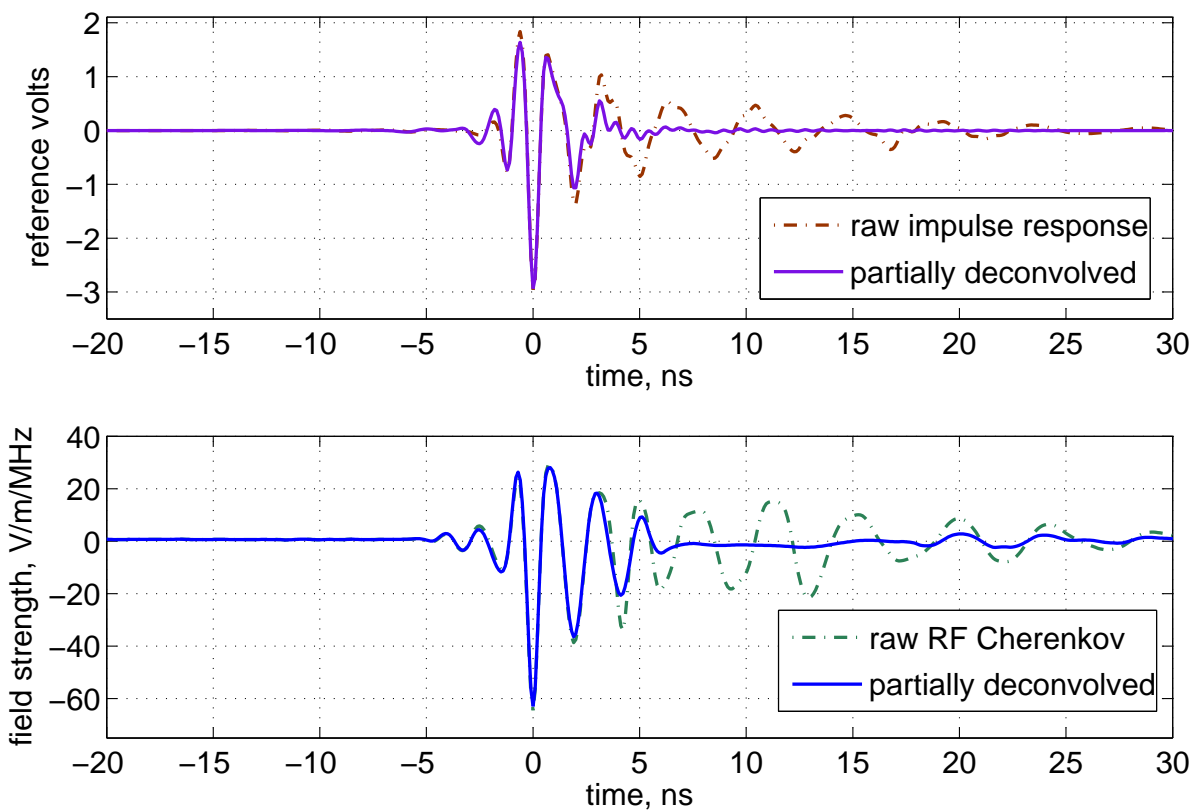

FIG. 17: Top: measured ANITA quad-ridge horn impulse response. Dashed lines include the strong nonlinear group-delay component near the horn low-frequency cutoff, and the solid line removes this low-power component using wavelet deconvolution, for better clarity. Bottom: waveform measured by ANITA antennas during SLAC calibration using direct Askaryan impulse signals from ice; dashed and solid lines are the same as in the top pane of the figure.

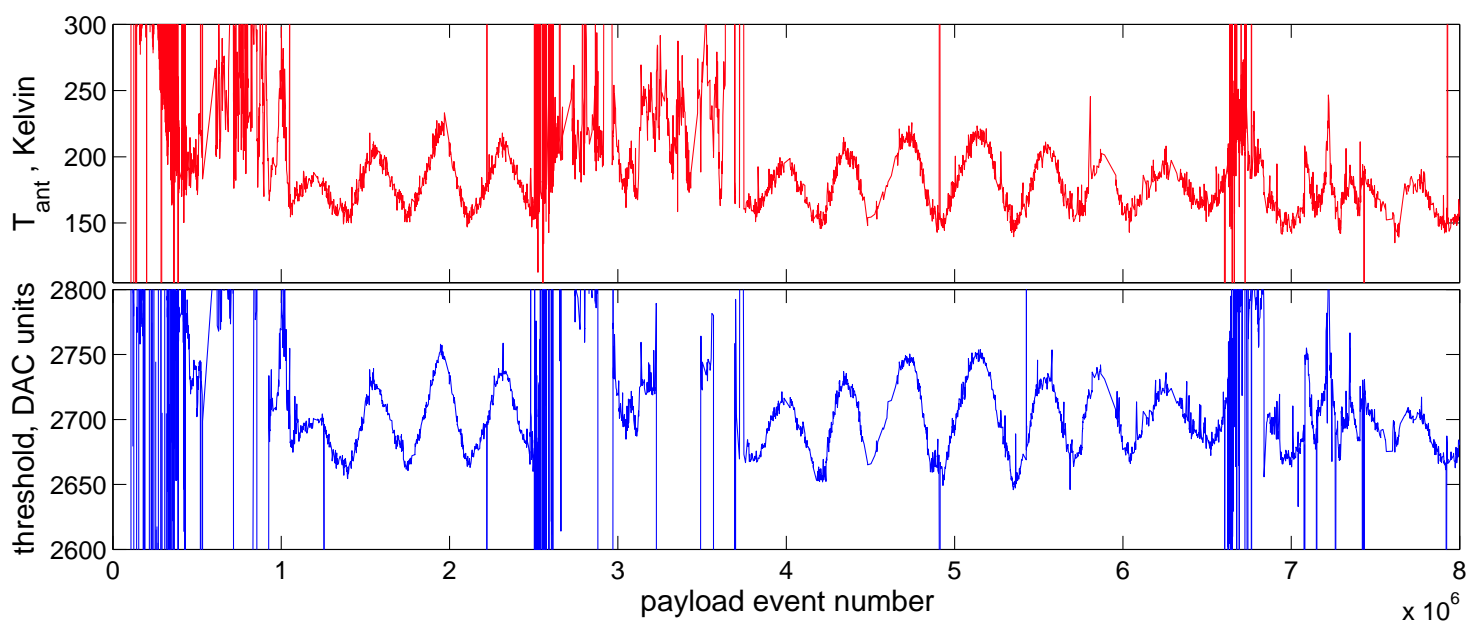

FIG. 18: Top: Antenna effective temperature vs. event number (at an average rate of about $4.5 \mathrm{~Hz}$ ) during the ANITA flight. The effective temperature is the average over the antenna beam including $230 \mathrm{~K}$ ice and $5-100 \mathrm{~K}$ sky, with varying contributions from the Sun and Galactic Center which produce the diurnal modulation. Modulation due to free rotation of the payload over several minute timescales has been averaged out. Bottom: The noise-riding antenna average threshold is shown on the same horizontal scale, showing the servo response between the instrument threshold and the apparent noise power. This noise-riding approach was used to retain a relatively constant overall trigger rate, giving stability to the data acquisition system.

where $k$ is Boltzmann's constant, $T_{A N T}$ is the effective antenna temperature, which includes contributions from the apparent sky (or sky+ice in our case) temperature in the field of view, along with antenna mismatch losses; $T_{R C V R}$ is the additional noise added by the front-end LNA, cables, and filters, and second-stage amplifiers and other signal- 
conditioning devices, all of which constitute the receiver for the incident signal. $T_{R C V R}$, which is an irreducible noise contribution, is usually fixed by the system design, and is calibrated separately (c.f. Fig. Đ). It is typically stable during operation and can be used as a reference to estimate additive noise. In contrast $T_{A N T}$ may vary significantly during a flight and must be monitored to ensure the best possible sensitivity of the instrument.

Absolute monitoring of the sensitivity for ANITA was accomplished by scaling the measured total-band power from the calibrated noise figure of the front-end receivers. This power was separately sampled at several $\mathrm{Hz}$ for each of the input channels, using a radio-frequency power detector/amplifier based on the MAXIM Integrated Circuits MAX4003 device, calibrated with external noise diodes. The absolute noise power was also cross-calibrated by comparison with known noise temperatures for the galactic plane and sun which were in the fields-of-view of the antennas throughout the flight, modulated by the payload rotation and by diurnal elevation changes.

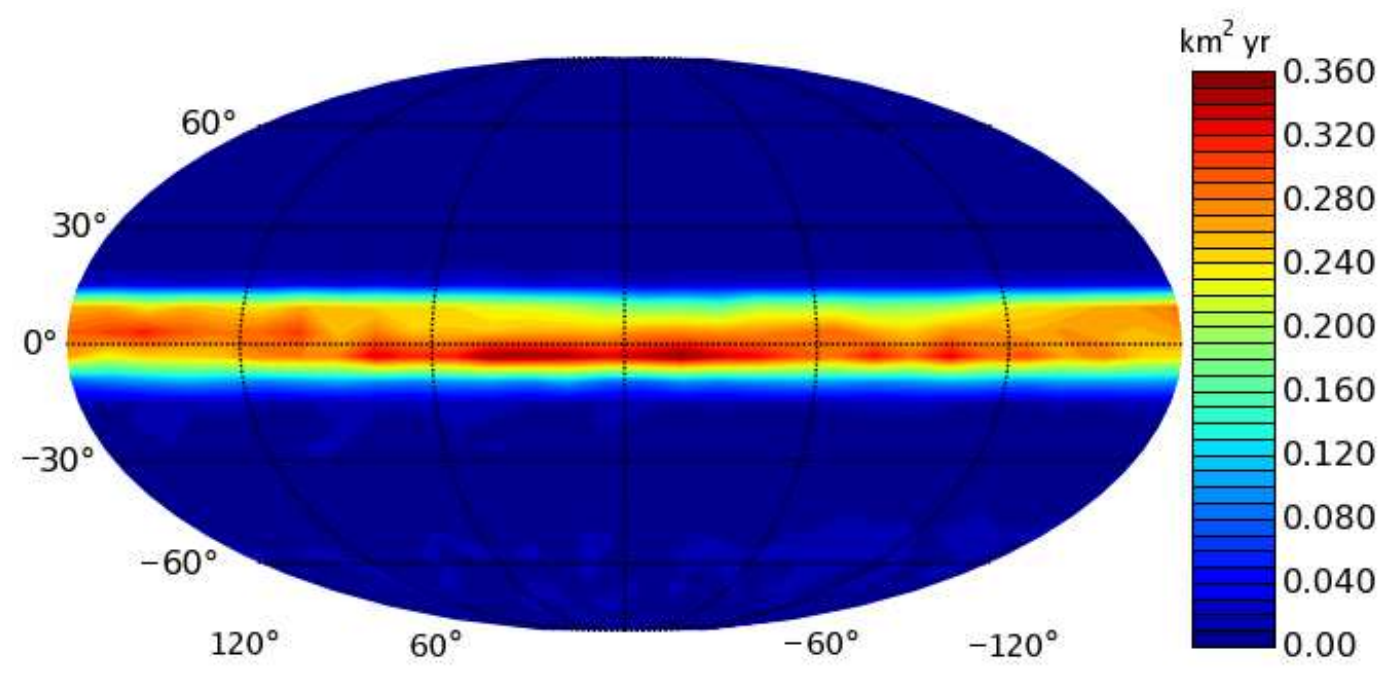

FIG. 19: A false-color map of sky exposure quantified by total neutrino aperture (here given for $E_{\mathrm{v}}=10^{20} \mathrm{eV}$ ) as a function of right-ascension and declination for the ANITA-1 flight.

A plot of the measured antenna temperature, derived from the RF power measurements, is shown in Fig. 18 as a function of the payload event number recorded during flight, which may be converted to payload time in seconds using a mean trigger rate of about $4 \mathrm{~Hz}$. Several features of this plot require explanation. A firmware error in a commercial Global-Position System (GPS) unit used for flight synchronization caused a series of in-flight timekeeping errors which led to a drift in payload time vs. real time; this was corrected in the data after the flight. Starting on day 21 (roughly event number 7M) of the flight, the flight computer began to experience anomalies which led to a series of reboots of the system, and increased deadtime. This behavior persisted until the termination of the flight after 35 days aloft. Beyond day 21, although we continued to take triggered data with an effective livetime of about $40 \%$, the environmental sensor data is fragmented and only a portion is shown here.

Values which rise off-scale on the vertical axis indicate periods when EMI dominated the system noise, as ANITA's trajectory came within the horizon of either McMurdo Station or Amundsen-Scott Station, both of which had strong transmitters in the ANITA bands. Other periods are largely quiescent as indicated by the plot; in particular the period between events $4 \mathrm{M}$ to $6.6 \mathrm{M}$. During these periods the observed antenna temperature is dominated by the $230 \mathrm{~K}$ ice in the lower portion of the antenna fields of view, averaged with the cold sky (3-10 K over our band) in the upper portion. The plotted values are averaged over all payload rotation angles (ANITA was allowed to freely rotate around its balloon suspension point). The observed modulation of the antenna temperature is thus due the diurnal changes in elevation angles of the Galactic Center and Sun, and has the expected amplitude for these sources over our frequency band, dominated by the nonthermal rise at the lower end of our band near $200 \mathrm{MHz}$, where the antenna fields of view are also the largest.

\section{B. Navigation performance}

Figure refGPS summarizes the navigation performance during the flight. Reconstruction of the ground position of any source that is detected during flight depends on accurate knowledge of the payload position and attitude. There 
are six degrees of freedom: latitude, longitude, altitude, orientation, pitch, and roll, and these are all determined via the ADU5 system on ANITA. Redundant information with somewhat lower precision is also obtained by a sun-sensor and magnetometer suite.
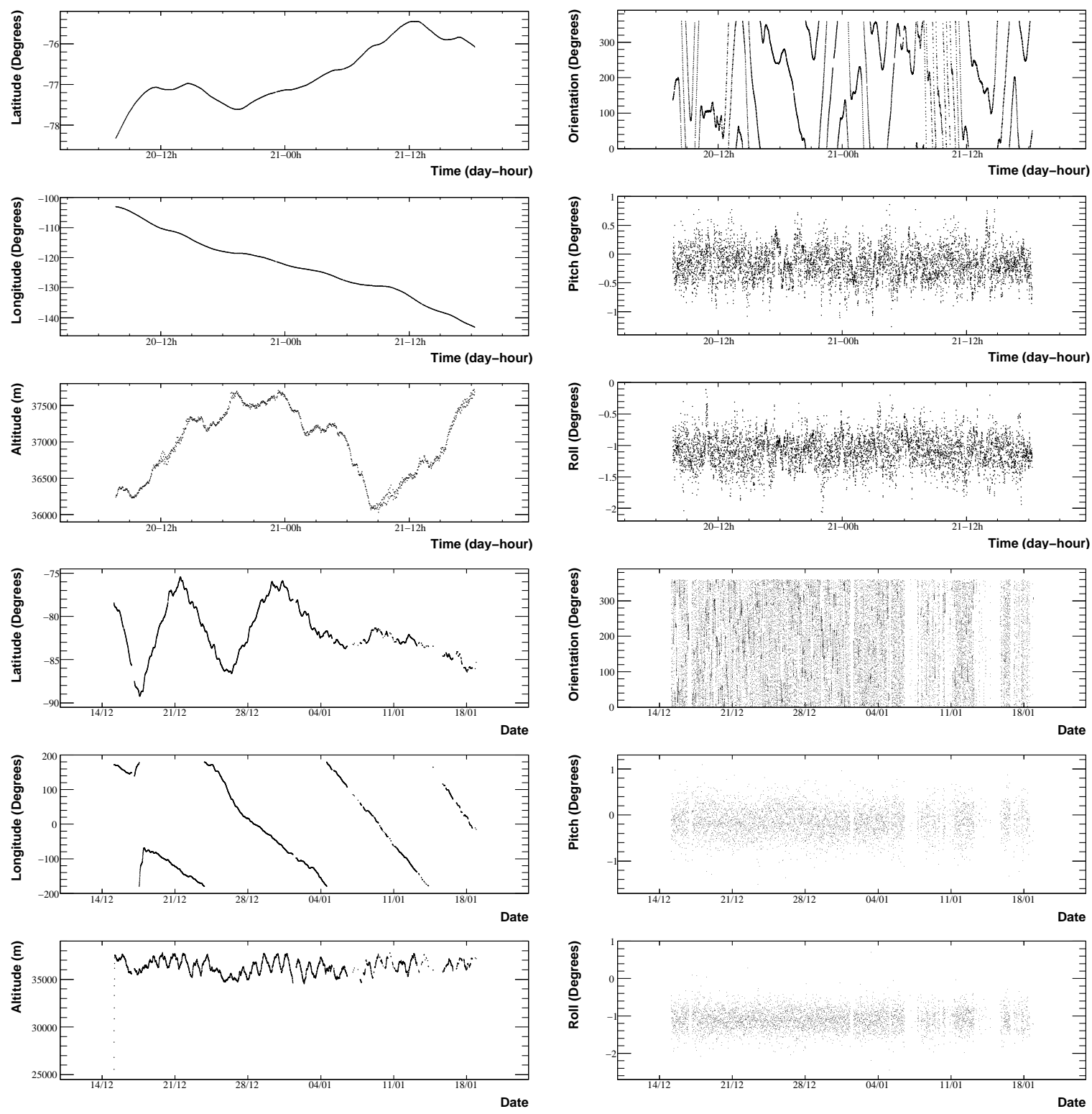

FIG. 20: A composite set of plots representing the in-flight performance of ANITA's navigation system. The left side plots are for geodetic position and altitude, and the right-side plots show the attitude parameters. The top 6 plots zoom in on a single data run, covering several flight days. The corresponding plots on the bottom half of the figure show the total time series for the full flight. Data for the orientation changes rapidly as the payload is allowed to freely rotate; thus the individual rotation cycles are difficult to see in the entire flight's data but show up clearly for the single data run.

Fig. 20 shows several features that illustrate important aspects of the navigation system. The payload was freelyrotating; that feature is illustrated by the orientation plots in the figure, which show the often rapid change of azimuth for the fixed reference coordinate system on the payload as it rotated with periods varying from minutes to hours during the flight. The payload pitch and roll are somewhat ill-defined for an azimuthally symmetric payload, but they do indicate that the reference plane of the GPS was slightly tilted, by about $1^{\circ}$ in the "roll" direction with respect to the horizontal. Overall, the performance of this navigation subsystem was within the flight requirements, and helped 
to ensure the accuracy of the direction reconstruction of impulsive radio sources detected throughout the flight.

\section{Flight path}
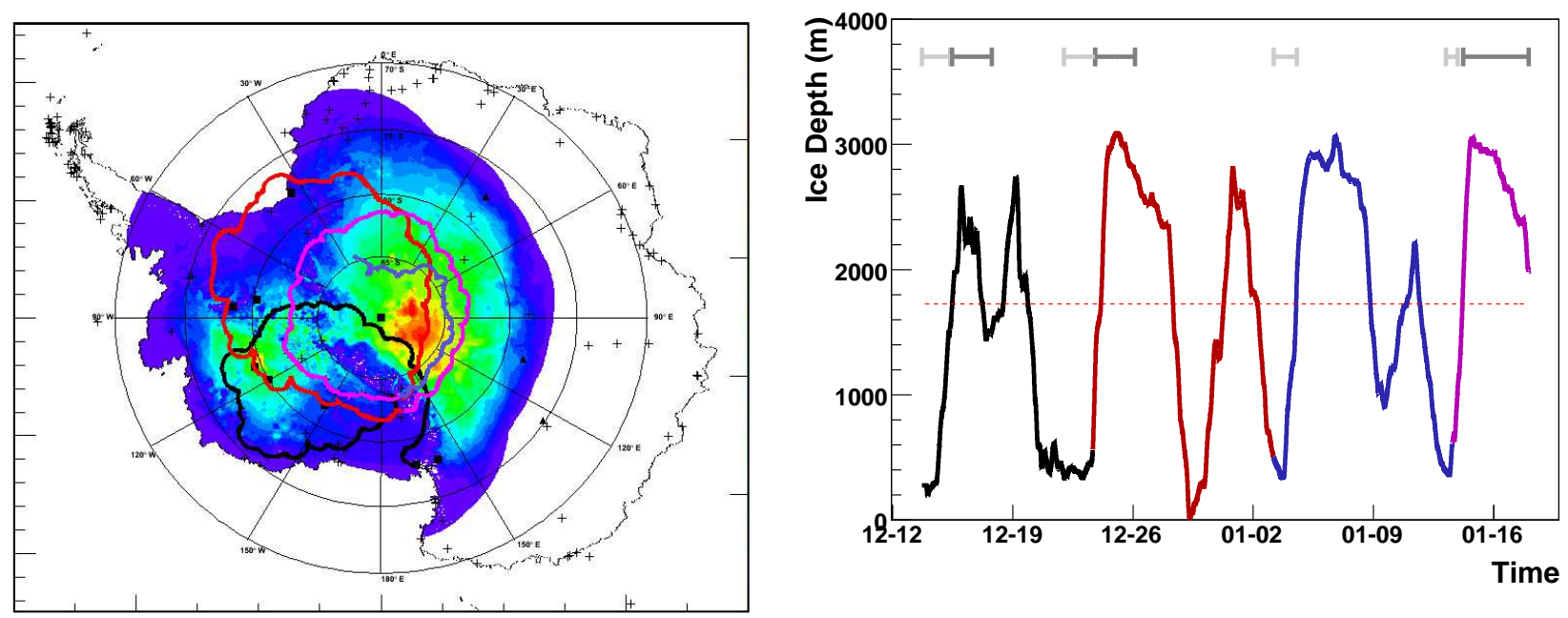

FIG. 21: Left: Flight path for ANITA-1, with depth of ice plotted. Only ice within the actual field-of-view of the payload is colored. Ice depths: red: $>4 \mathrm{~km}$; yellow: 3-4km; green:2-3km; light blue 1-2km, blue: $<1 \mathrm{~km}$. Right: average depth of ice within the payload field of view during the flight. Because the average includes all ice within the field of view, the minima and maxima of this curve do not correspond to the minima and maxima in the actual localized ice depths.

During the austral summer in the polar regions, the polar vortex usually establishes a nearly circumpolar rotation of the upper stratosphere, and payload trajectories are often circular on average, even over several orbits. During the austral summer of 2006-2007 however the polar vortex did not stabilize in a normal configuration, but remained rather weak and centered over West Antarctica. This led to an overall offset of the centroid of the orbital trajectories for ANITA-1, as seen in Fig. 21(left).

This anomalous polar vortex had two consequences for ANITA's in-flight performance: First, it led to a much larger dwell time in regions where there were higher concentrations of human activity, primarily McMurdo and AmundsenScott South Pole Stations. The presence of these and other bases within the field-of-view of the payload led to a higher-than expected rate of noise triggers and compromised the instrument sensitivity over a significant portion of the flight. Second, the higher-than-normal dwell time over the smaller and thinner West Antarctic ice sheet, rather than the main East Antarctic ice sheet, led to a lower overall exposure of ANITA to deep ice. The first consequence is apparent in Fig. 18, where it is evident that as much as $40 \%$ of the flight suffered some impact on sensitivity due to the presence of noise sources in the field of view. The second consequence is quantified in Fig. 21(right) where we plot the field-of-view-averaged depth of the ice along the flight trajectory, along with the overall average. Despite the large dwell time over West Antarctica, ANITA still achieved an average depth of ice during the entire flight of more than $1500 \mathrm{~m}$.

\section{Sky coverage}

In Fig. 19 we show a false-color map of the relative neutrino sky exposure in Galactic coordinates for ANITA's flight. The exposure is determined both by the geometric constraints of the flight path, and by the neutrino absorption of the earth, which limits the acceptance to within several degrees of the horizon at any physical location over the ice. It is evident from this plot that ANITA had significant exposure both to the galactic plane and out-of-plane sky, and although the total sky solid angle covered is only of order $10 \%$, the exposure is adequate to constrain a diffuse neutrino flux component, as well as provide some constraints on point sources within the exposed regions. 

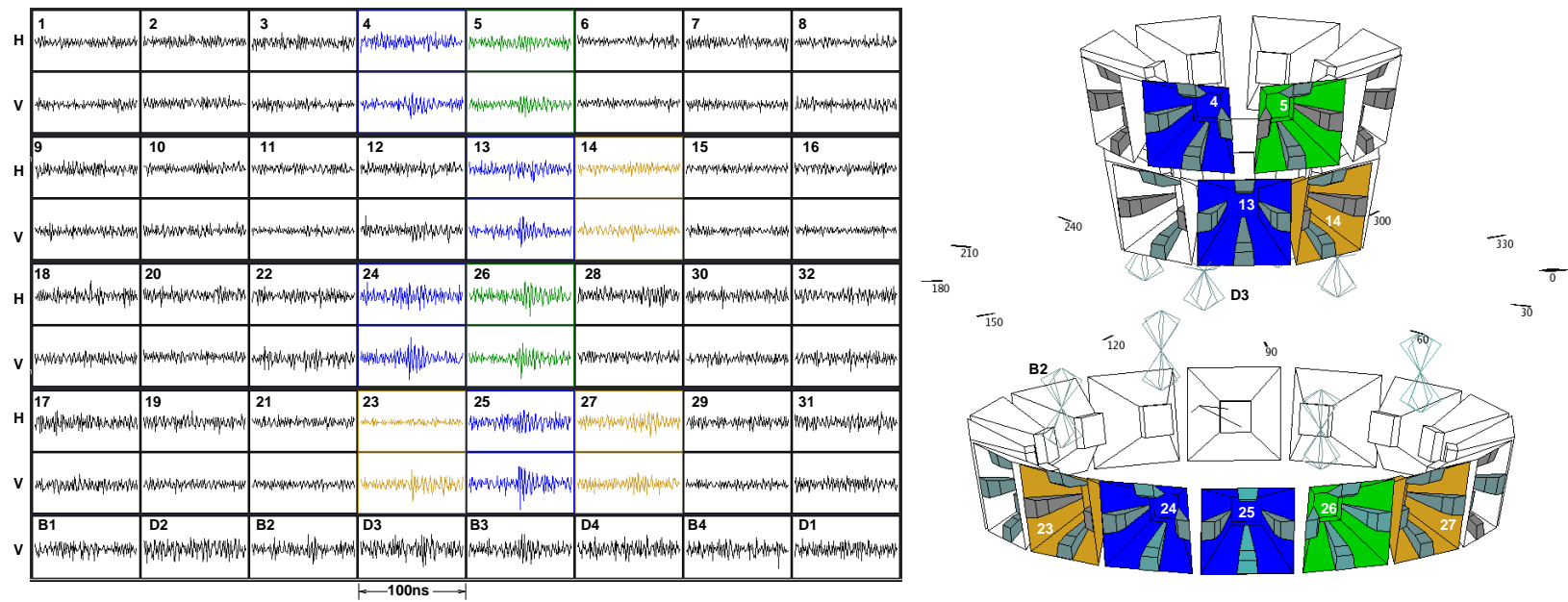

FIG. 22: Plot of an example triggered event (attributed to interference from a West Antarctic encampment) during the ANITA flight, as viewed with the realtime data viewers, both as a set of the entire payloads waveforms (left) and the trigger antenna geometry (right). Colors indicate the trigger hierarchy: yellow indicates L1 trigger, green L2, and blue a global L3 trigger.

\section{E. Data quality and volume}

ANITA recorded approximately 8.2 M RF-triggered events during the 35 day flight, and for each of these events all of the antennas and different polarizations were read out and archived to disk. The system was able to maintain a trigger rate of between 4 and $5 \mathrm{~Hz}$ during normal operation without any significant loss, and this rate, which was determined by the individual antenna thresholds, met the pre-flight sensitivity goal for periods when the payload was not in view of strong anthropogenic noise sources. In practice, less than $1 \%$ of all recorded triggers do actually arise from a coherent plane-wave impulse arriving from an external source. The vast majority are random thermal-noise coincidence events, which are recorded to provide an ongoing measure of the instrument health, and separate validation of the sensitivity.

The performance of the data acquisition system for non-thermal events is best summarized by showing a typical impulsive event as recorded by the system and displayed by the in-flight monitor on the ground receiving computer system. A partial display of event 7767328 is shown in Fig. 22. On the left are the recorded waveforms for this event, which clearly show an impulsive signal superposed with thermal noise on the left, where all 72 antenna signals are displayed. Vertical columns are organized in azimuth around the payload, with horizontal rows providing both the vertical and horizontally polarized signals from the vertically offset antenna rings of the payload. The physical geometry of the arriving signal is indicated in the payload view to the right, where colors indicate which ones produced the actual triggers and at what level. These colors also match up with the colored waveforms on the left.

These raw-data waveforms, while appearing somewhat noisy, actually have quite high information content, with up to ten usable signals detectable above thermal noise even in the raw data in this event. After processing, which typically includes a matched-filter correlation designed to optimize the SNR of broadband impulsive events, such signals are used to provide strong geometric constraints on the arrival direction of the event, as we will detail in a later section.

\section{F. Ground-to-payload calibration pulsers.}

The ANITA instrument requires the highest possible precision in reconstructing the arrival direction of any incoming source event so that it may reject any impulses that might mimic neutrino signals but arise from anthropogenic electromagnetic interference. In addition, ANITA must verify the in-flight sensitivity of the trigger system to external events. To accomplish this, we developed a ground-based calibration approach which relied on pulse transmitters which could be directed at the payload from several sites during the flight, and used to establish both pointing accuracy and sensitivity. Here we describe the performance and results using these systems. 


\section{Description}

We deployed four ground-to-payload transmitter antennas at two distinct sites during the 2007-2008 ANITA flight. The antennas transmitted radio impulses to the payload in order to verify instrument health and provide a sample of neutrino-like signals for testing our analysis codes. A field team traveled to Taylor Dome $\left(77^{\circ}, 52^{\prime} \mathrm{S} ; 150^{\circ}, 27^{\prime} \mathrm{E}\right)$ and operated a surface quad-ridged horn antenna and a borehole discone-type antenna in a 100 meter deep borehole, about $30 \mathrm{~m}$ below the firn-ice boundary at Taylor Dome. Results from concurrent Taylor Dome experiments by the ANITA team on ice properties at this site are presented in a separate report [52].

The team at Williams Field at the LDB launch site operated a surface quad-ridged horn antenna and a discone in a 26 meter deep borehole. All four antennas transmitted a kilovolt-scale impulse $\approx 500$ ps wide with a flat frequency spectrum. The impulse was chosen to be as close as possible to theoretical predictions of a neutrino Askaryan signal. The surface antenna at McMurdo could adjust the amplitude and polarization of the impulse and occasionally transmitted brief continuous wave (CW) signals. The surface antennas at both McMurdo and Taylor Dome were quad-ridged horn antennas identical to those on the ANITA payload. The borehole antennas were both discone antennas designed and built at the University of Hawaii for use in ice.

The payload used two methods of detecting impulses: the standard RF trigger and a forced trigger using a preset time offset relative to the GPS pulse-per-second (PPS). Anthropogenic EMI from McMurdo restricted our ability to operate in RF trigger mode when the base was above the horizon. Despite this noise, we were able to use RF triggers for several hours during which noise levels were low. Both as a supplement to the RF triggered events and also for easy event identification, we timed the McMurdo antennas' transmissions such that the signals always arrived at the payload at a constant time offset from the GPS PPS. These PPS triggers allowed us to record calibration signals during periods that would have otherwise been unusable due to anthropogenic noise.
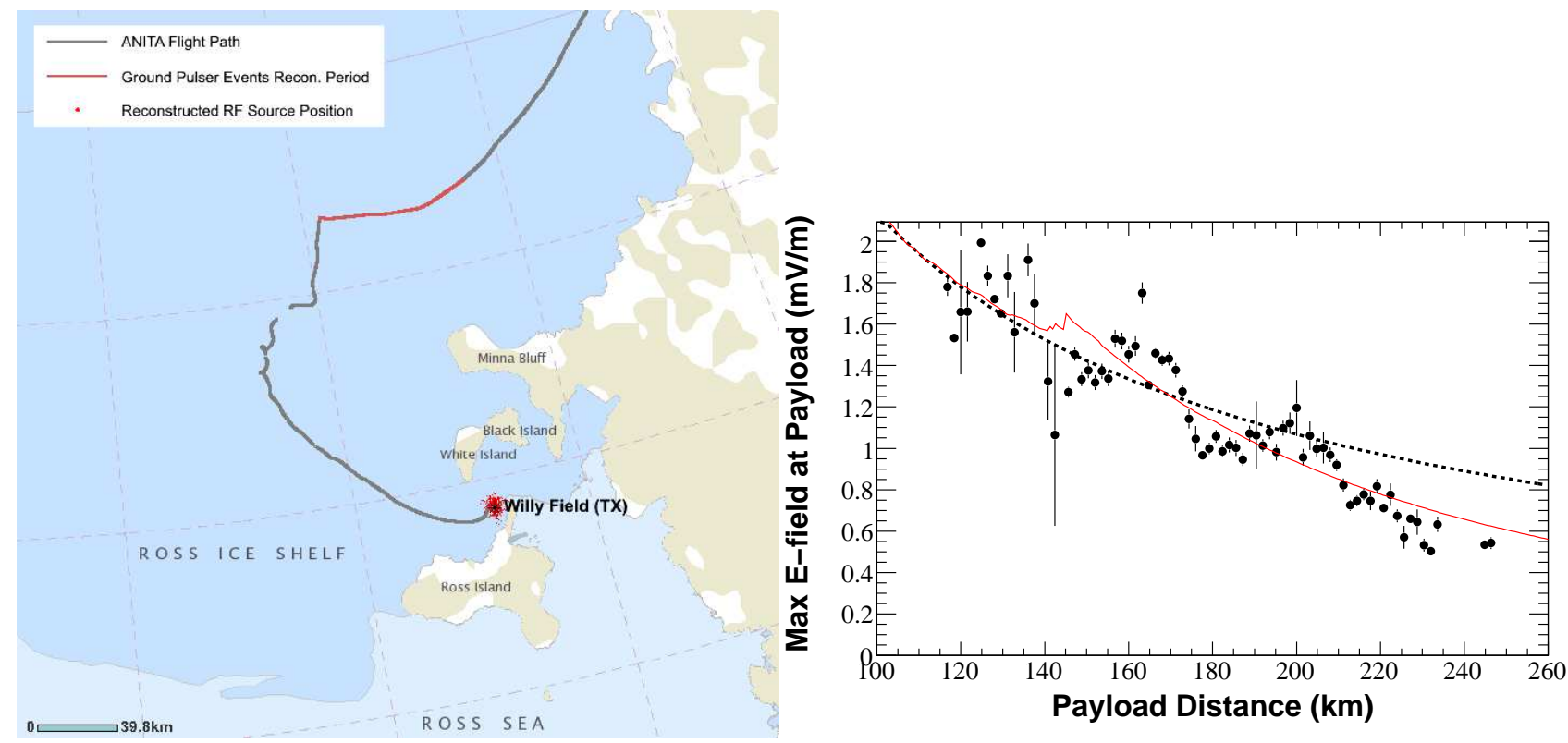

FIG. 23: Left: The flight path of ANITA in the first day after launch, with the reconstructed positions of signals from McMurdo ground calibration systems. Red dots are projected positions of the reconstructed events, the line is a trajectory of the ANITA flight, and the red part of the line is for the flight segment for this reconstruction. Right: Distance of the ANITA payload from Willy Field versus the maximum E-field of the pulse received from the McMurdo ground pulser borehole antenna. The solid line is a fit to a $1 / \mathrm{r}$ curve multiplied by an angle-dependent Fresnel factor. The dashed line is a pure $1 / \mathrm{r}$ curve with arbitrary normalization for reference. (The overall normalization agreed with the absolute scale to about $10 \%$ ). 

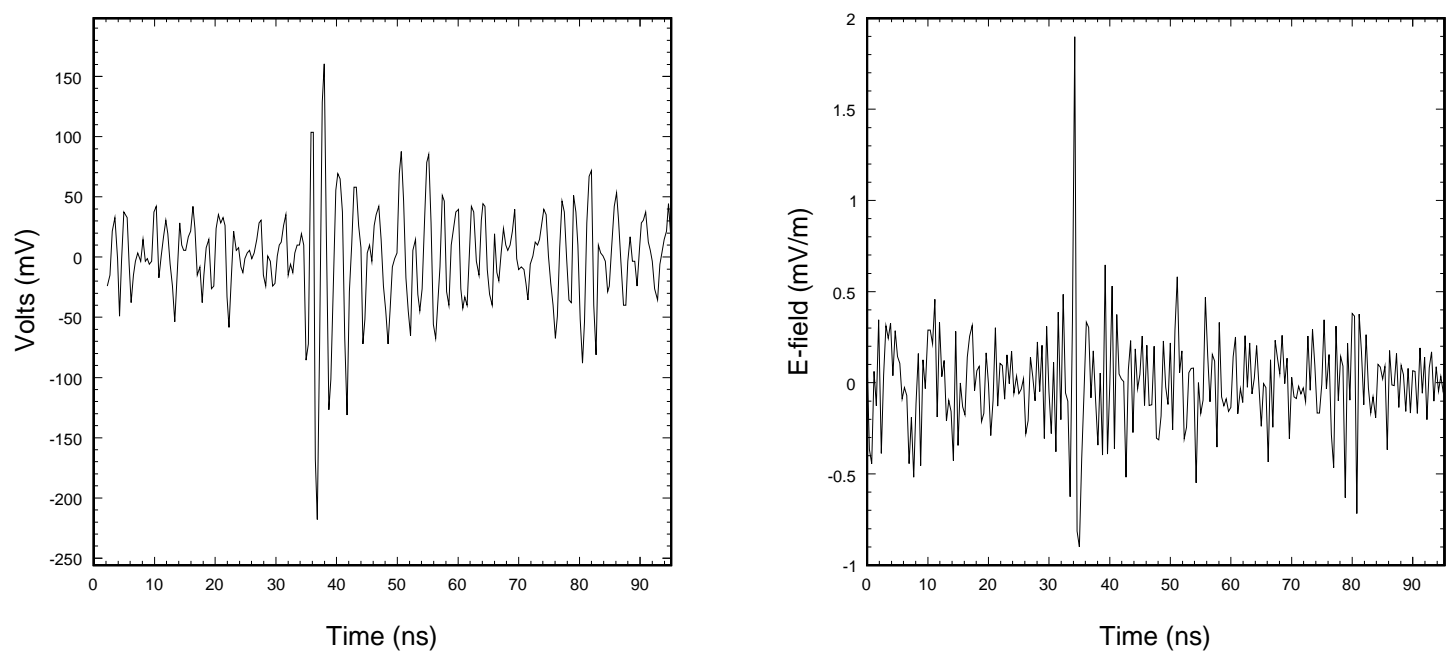

FIG. 24: Left: A ground calibration pulse from the McMurdo surface antenna, recorded during the 2006-2007 ANITA flight. Right: The same pulse, with instrument gains and group delays removed.

\section{Uses of Ground Calibration Pulses}

The ANITA payload successfully recorded tens of thousands of ground pulser signals. ANITA detected signals from the borehole at Williams Field at ranges up to $260 \mathrm{~km}$; Fig. 23 shows a portion of the first day's flight path with a segment of the path from which events were efficiently reconstructed due to a lull in surface noise activity near McMurdo station. The surface antennas at McMurdo and Taylor Dome could be seen from even further away. ANITA's flight path took it no closer than about $250 \mathrm{~km}$ to Taylor Dome, and the limited power of the borehole pulser there, combined with the higher loss in the longer cable needed to accommodate the much deeper borehole there made it difficult to detect the Taylor Dome borehole pulses, as most were below the ANITA detection threshold. However, of order 20 of these borehole impulses were detected, all very close to the instrument hardware threshold.

Figure 23 shows the maximum E-field strength from the McMurdo borehole antenna at the location of the ANITA instrument, as a function of distance. More than 10,000 events are plotted here. The line is the predicted peak of the E-field strength, taking into account Fresnel factors at the payload's location and the distance of the payload from the transmitting antenna. This plot illustrates the utility of a calibration source for measuring long-term stability of instrument gain. Changes in ANITA's gain are limited to the $10 \%$ level over the day covered by the data in Figure 23 , and these constraints on the variation include the effects of any variation in surface roughness in the surface Fresnel zone of the borehole transmitter antenna.

We perform a final adjustment of antenna timing using the plane wave source. We use it to check the impulse response measured in the lab.

The amplifiers, filters, and other components of the flight system introduce frequency-dependent gains and group delays in the measured waveforms. Using lab measurements of the instrument's RF properties, we can deconvolve the instrument's impulse response from our data, retrieving the E-field that was incident upon our antennas. We perform a final adjustment of antenna timing using the plane wave source. We use it to check the impulse response measured in the lab. Once again, the ground calibration data allows us to test this process, since we know what the incoming signal was. Figure 24 is an example of such a test. The deconvolved signal is much sharper. In fact, the deconvolved signal is the impulse response of an ANITA quad-ridged horn antenna - which is also the result of transmitting an impulse such as the one used in the ANITA ground calibration system.

This deconvolution process is also useful in establishing the coherent transmission properties of the ice, both internally as the pulse must pass through the ice bulk, and interface transmission as it passes through the ice surface which is potentially rough on scales that may not be negligible compared to the wavelength of the radiation. This propagation test was a focus of our calibration system at Taylor Dome. In Fig. 25 we show a pair of waveforms, the left one the raw waveform of a Taylor Dome pulse, and the right one the deconvolved impulse which is highly coherent. The fact that this impulse was transmitted of order $150 \mathrm{~m}$ (e.g., the slant depth to the surface exit point) through the ice and firn, 

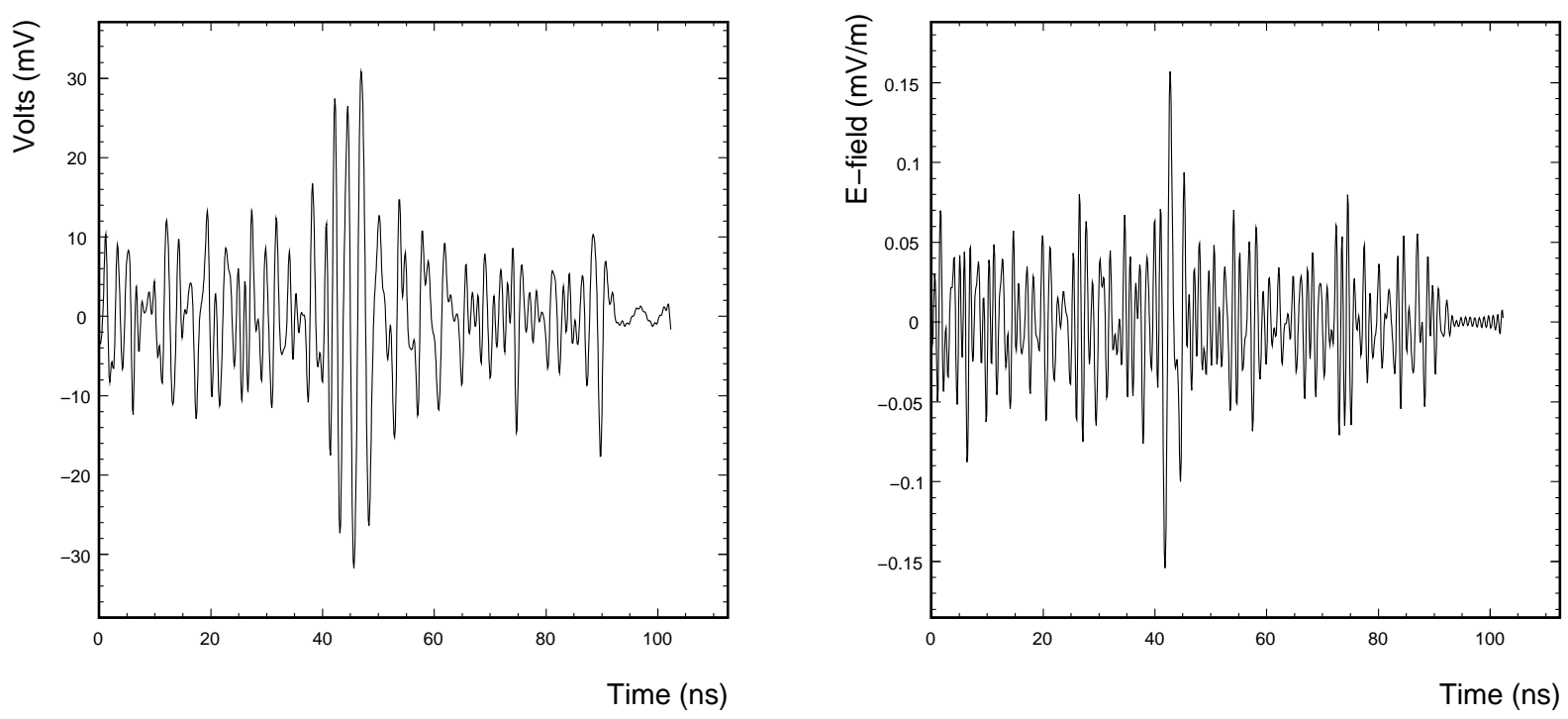

FIG. 25: Left: Raw impulse from Taylor dome borehole. Right: Deconvolved impulse from the same waveform data, showing that the pulse remains highly coherent despite the propagation effects of the ice, firn, and surface through which the impulse was transmitted.

along with the firn surface, with no apparent loss of coherence (which would appear as possible distortion or splitting of the pulse) provides good evidence that the methodology employed by ANITA is effective in detection of Askaryan impulse events from high energy showers.

\section{G. Timing/Pointing analysis}

\section{ANGULAR RECONSTRUCTION}

Angular reconstruction of RF signals is a crucial part in the ANITA data analysis. It provides powerful rejection of incoherent thermal noise events which comprise $\geq 95 \%$ of the data set, anthropogenic RF events from existing bases and field camps, and radio Cherenkov events from air showers. If ANITA observes candidate neutrino events, angular reconstruction will be important for first-order neutrino energy estimation, for providing directional information, propagation distance $(r)$ for $1 / r^{2}$ corrections, and the RF refraction angle on the ice-air boundary for the Fresnel correction.

Although ANITA's trigger uses circularly polarized signals to provide an unbiased sensitivity to both linearly polarized events, the signals are separately recorded as their horizontally- and vertically- polarized waveform components. Our Monte-Carlo analysis has indicated that the neutrino signals of interest tend to be strongly favor vertical over horizontal polarization, and thus for simplicity we analyze the H-pol and V-pol waveforms as separate data sets. In the following description, the analysis steps are applied in parallel to both the polarizations independently. All steps of the analysis are optimized for either a $10 \%$ fraction of the data, or the full data set with the payload orientation kept unknown till after the analysis was complete. This is done to eliminate analyst bias in developing the data cuts. The separation of the data streams into H-pol and V-pol sets also helps to eliminate any selection bias, since the cuts are applied in identical fashion between the two sets.

\section{A. Cross Correlation}

Direction reconstruction of the RF signal uses the arrival timing difference $(\Delta t)$ information between pairs of antennas. $\Delta t$ is determined by using a cross-correlation technique between recorded waveforms of the antennas. The cross correlation coefficient $(R)$ is defined by 


$$
R=\frac{\sum_{t}\left(V_{i}(t)-\bar{V}_{i}\right)\left(V_{j}(t+\delta t)-\bar{V}_{j}\right)}{\sqrt{\sum_{t}\left(V_{i}(t)-\bar{V}_{i}\right)^{2}} \sqrt{\sum_{t}\left(V_{j}(t+\delta t)-\bar{V}_{j}\right)^{2}}} .
$$

Here, $V(t)$ is a recorded voltage value at a time bin $t$ and $\delta t$ is time delay. The $i$ and $j$ denote channel numbers. For fast calculation, the mean voltage $\bar{V}$ is first subtracted to normalize the mean to zero. In order to minimize the effects any anthropogenic continuous wave interference, we use a limited time window of the signal waveform of the antenna $i ;\left|t-t_{\text {peak }}\right| \leq 15 \mathrm{~ns}$, where $t_{\text {peak }}$ is the time bin in which the maximum peak voltage, $V_{\text {peak }}$, exists.

For a given pair of antennas $i$ and $j$, we search for the time lag $\Delta t$ which gives the maximum correlation coefficient, $R_{\max }$, while varying $\delta t$ within $\pm 25 \mathrm{~ns}$. In order to obtain more precise time resolution than the $0.38 \mathrm{~ns}$ sampling time interval of the ANITA digitization system, we use interpolated waveforms with a $7.6 \mathrm{ps}$ bin width which is a five-fold oversampling of the original waveforms.

\section{B. Group Delay Calibration and Time Resolution}

A precise timing measurement using the cross correlation technique allows an accurate calibration of the fixed group delay for each channels. Although all fixed group delays were also measured in the laboratory before the flight, calibration during the flight was performed to reduce systematic uncertainties such as temperature dependence and possible antenna coordinate changes after the launch. We measured all relative group delays using the impulse signal transmitted from ground based calibration pulser system ??[reference for ground pulser here]. A group delay difference $\left(\delta T^{i j}\right)$ between two channels of $i$ and $j$ is obtained by comparing observed timing difference $\Delta t_{\text {obs }}$ and its expectation $\Delta t_{\mathrm{exp}}$;

$$
\delta T^{i, j}=T_{\text {delay }}^{i}-T_{\text {delay }}^{j}=\Delta t_{\text {obs }}^{i j}-\Delta t_{\text {exp }}^{i j}
$$

where the $\Delta t_{\exp }^{i j}$ is given by the difference of RF propagation times from the coordinate of transmitter to each receiver antenna.

Figure 26 shows distributions of time differences $d t=\Delta t_{\mathrm{obs}}-\Delta t_{\mathrm{exp}}$ after applying the group delay correction for all channels. Obtained time resolutions for the upper/lower antenna pairs in the same $\Phi$-sector is $\sigma_{\text {same } \phi}=47 \mathrm{ps}$. Because of additional jitter due to the intrinsic jitter of time synchronization between different SURF boards allocated to different $\Phi$-sectors ??, a slightly worse time resolution of $\sigma_{\text {diff } \phi}=66 \mathrm{ps}$ is obtained for antenna pairs in different $\Phi$-sectors.
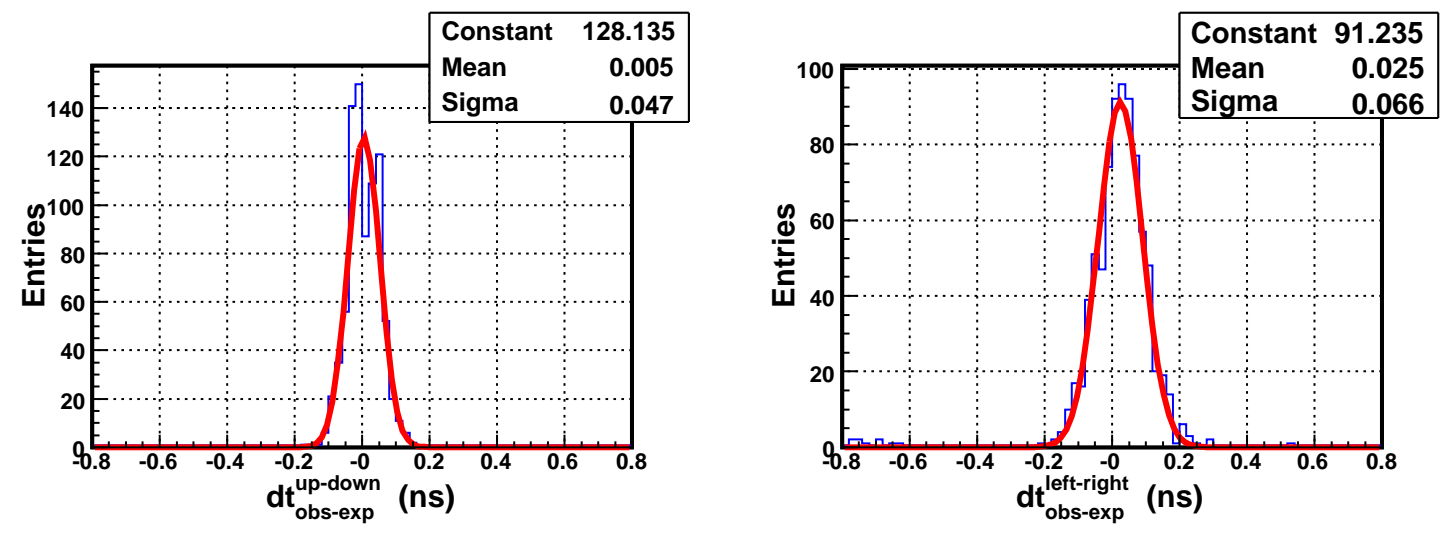

FIG. 26: $d t$ distributions: Upper/lower antenna pairs in the same $\Phi$-sector (Left) and antenna pairs in different $\Phi$-sectors (Right).

Once the calibrations are applied, a first order method to establish the presence of a coherent signal source is to sum the two-dimensional cross-correlated intensities of all baseline pairs into a summed-power interferometric image, as 
shown in Fig. 27. for one of the surface antenna pulses from Taylor Dome. In this procedure each pair of antennas gives a fringe across the sky at an angle corresponding to the baseline direction, and the fringes sum together with the greatest strength at the source location. The virtue of this method is that it can also be used to identify the location of sidelobes in the image, so that later quantitative fitting of the source location can be tested to ensure that it has not produced a misreconstruction at one of the sidelobe locations.

Such an image is highly analogous to a "dirty map" in radio astronomical usage, and a reduction in sidelobes is possible with further image processing. However, we have found in general that for this type of "pulse-phase interferometry," these maps are adequate since all sources are unresolved for ANITA. We have also studied phase closure methods as applied to our data, and we find that further work in this area is justified and may yield even more robust methods for this type of source imaging.

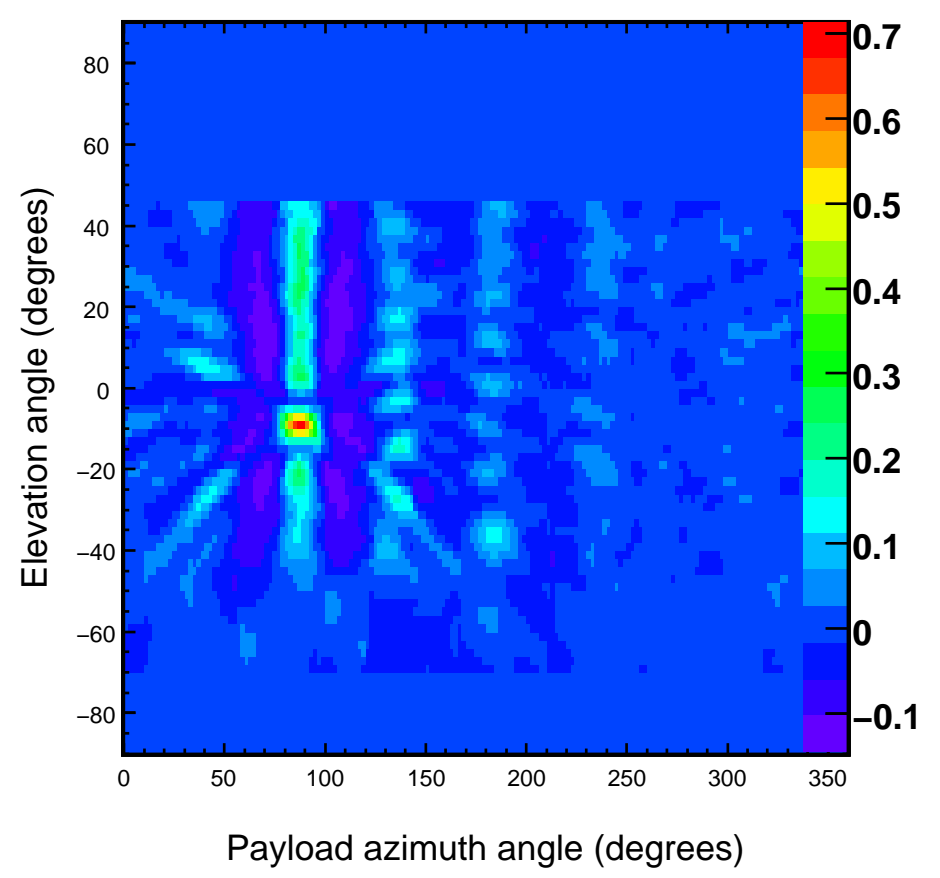

FIG. 27: Interferometric image of a single impulsive event from the Taylor Dome ground calibration pulser.

\section{Good event selection and hit cleaning}

For the angular reconstruction, we use antennas in three $\Phi$-sectors around the maximum $\Phi$-sector where an average peak voltage of the upper and lower antennas is the highest among all $\Phi$-sectors. The upper antenna on the maximum $\Phi$-sector is used for a reference channel for the cross correlation. Channels having anomalous or outlying timing information are not used for the reconstruction. We compare $\Delta t_{\mathrm{obs}} \mathrm{s}$ for all possible baselines (15 possible) in the 6 antennas around the maximum $\Phi$-sectors, then we regard the channel as an isolated hit if multiple baselines associated with this channel have more than $12 \mathrm{~ns}$ deviation.

A selection for good events is applied before the angular fit in order to speed up analysis and reduce misreconstructions. We require the number of good antenna hits $N_{\text {hit }}>5$, the peak voltage to be $V_{\text {peak }}>35 \mathrm{mV}$, and a signal to noise ratio to be $\left(S N R=V_{\text {peak }} / V_{r m s}>3.5\right)$ for both of upper and lower antennas in the maximum $\Phi$-sector, where the $V_{r m s}$ is a root mean square of voltage obtained in the first third of the recorded waveform which is well-separated from the signal region near the center of the recorded waveform. Note that the SNR recorded in the digitizer waveform is generally lower than the SNR of the analog RF trigger, since there are additional insertion losses associated with the digitizer itself, as described in a previous section. 


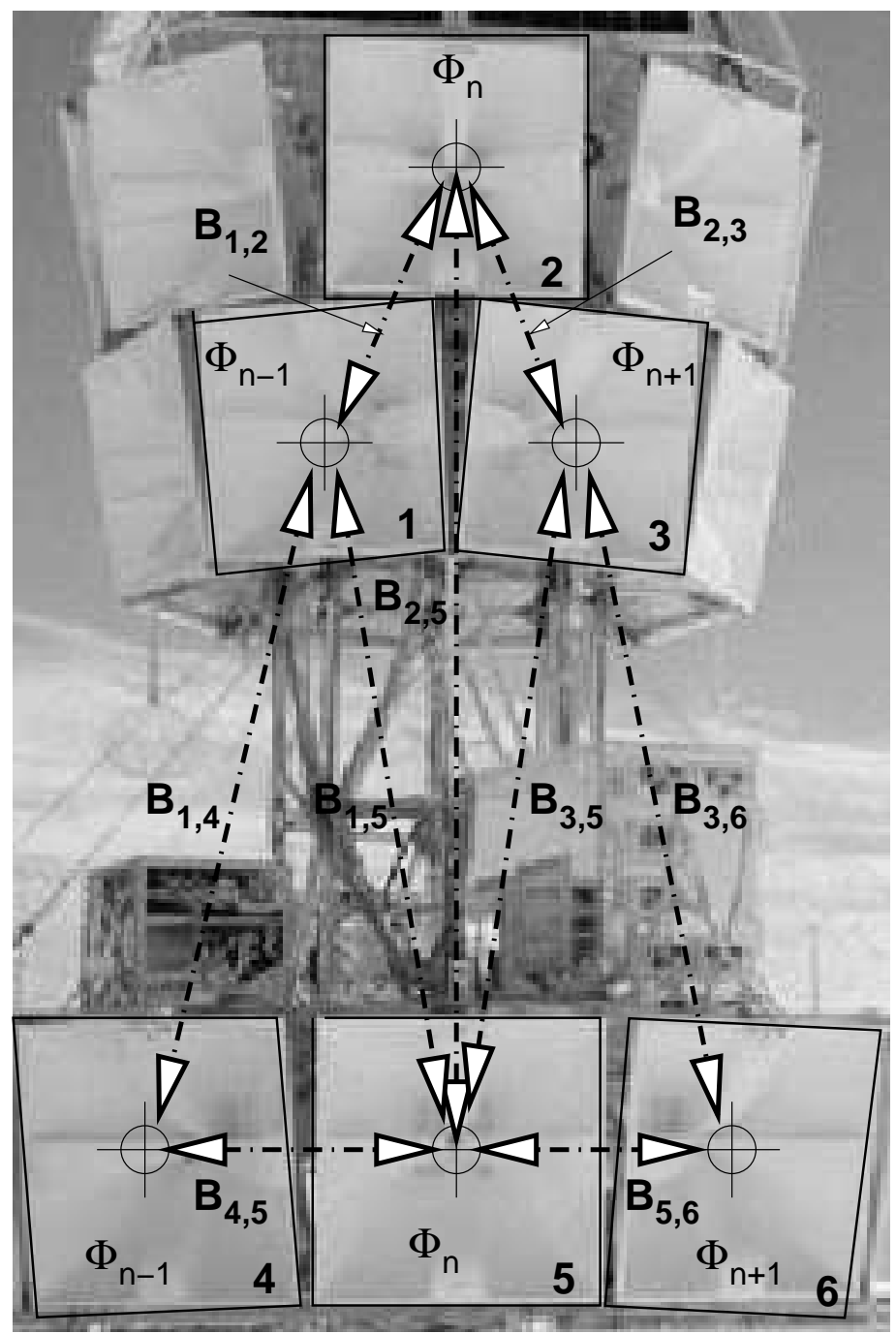

FIG. 28: Illustration of basic set of baselines for the angular fit. Not all of the fifteen possible baselines for 6 antennas are shown.
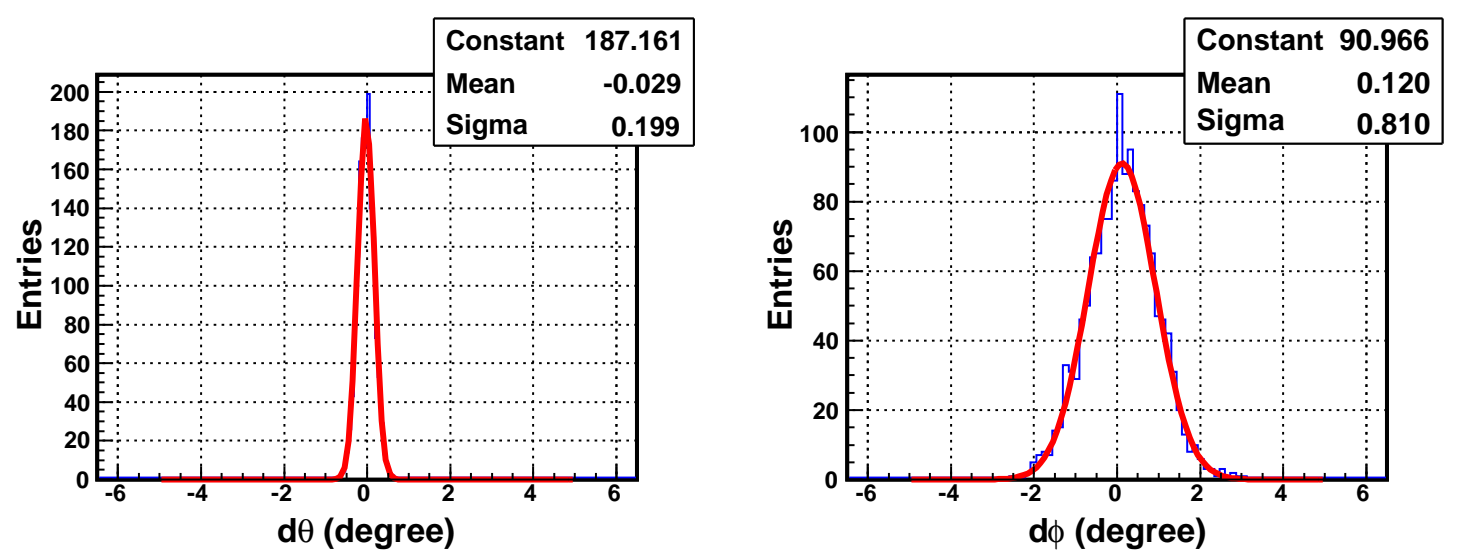

FIG. 29: Angular resolution: $d \theta$ distribution (Left) and $d \phi$ distribution (Right) 


\section{Angular Fit}

The number of timing baselines $N_{\text {base }}$ we use in the fit is $N_{\text {hit }}-1$. A selection from among the fifteen possible baselines for six antennas is made in a way to minimize timing uncertainties and correlations between the baselines. Figure 28 illustrates some of the possible baselines for an event with a phi-sector containing the maximum possible vertical baseline; either adjacent phi sector would involve a shorter principal baseline paired with longer adjacent ones.

We perform a $\chi^{2}$ fit in order to find the direction of the RF signal. It minimizes

$$
\chi^{2}=\sum_{k}^{N_{\text {base }}}\left(\frac{\Delta t_{\text {obs }}^{k}-\Delta t_{\text {hypo }}^{k}(\theta, \phi)}{\sigma_{k}}\right)^{2} .
$$

Here, the $N_{\text {base }}$ is the number of baselines, the $\Delta t_{\mathrm{obs}}^{k}$ is timing difference of the antenna channels participating in baseline $k$, the $t_{\text {hypo }}^{k}(\theta, \phi)$ is its expectation for the RF signal with a given hypothesis of a plane wave direction $(\theta, \phi)$, and the $\sigma$ is the time resolution of the corresponding baseline. Since the time resolution is varied depending on the signal strength, we use $S N R$ dependent time resolution;

$$
\sigma=\sigma_{S N R}^{\mathrm{ch} 1} \oplus \sigma_{S N R}^{\mathrm{ch} 2} \oplus \sigma_{\mathrm{sys}},
$$

where the $\sigma_{S N R}$ is a single channel timing error caused by thermal noise and the $\sigma_{\text {sys }}$ is intrinsic system error. The ch1 and ch2 denote two channel numbers involved with the baseline. A functional form of $\sigma_{S N R}$ is obtained by a Monte Carlo simulation (MC) study of a thermal noise effect on time resolution of the impulsive signal. We use the measured time resolutions for $\sigma_{\text {sys }}$, which can be either $\sigma_{\text {same } \phi}$ or $\sigma_{\text {diff } \phi}$ depending on the geometry of the baseline.

We perform 10 iterations of fits to reduce misreconstructions caused by trapping of the solution in local minima of $\chi^{2}$. In each iteration, initial fit parameters of $(\theta, \phi)$ are uniformly varied within $\theta>90^{\circ}$ and $\phi_{\max }-25^{\circ}<\phi<$ $\phi_{\max }+25^{\circ}$, where the $\phi_{\max }$ is an azimuth angle of the boresite of the antenna in the maximum $\Phi$-sector. We require $\chi^{2}<4$ for a well reconstructed event.

Figure 29 shows angular resolution with the ground-based calibration pulsers. The $d \theta(d \phi)$ is a deviation of zenith (azimuth) angle from expected value. Achieved resolutions are $0.2^{\circ}$ and $0.8^{\circ}$ respectively. The worse resolution of $d \phi$ than $d \theta$ is due to the shorter length of baselines in the $\phi$ direction and the worse time resolution of the inter- $\phi$ baselines.

\section{E. Misreconstruction Rejection}

A misreconstruction is a fit result that deviates from the expected source direction while still having a good fit quality or low $\chi^{2}$. Misreconstruction is one of the most important potential background sources for the neutrino search in the ANITA experiment, because a misreconstructed event of anthropogenic origin from, for example, a known encampment, might become a neutrino candidate.

The main sources of the misreconstruction are incorrect timing information and a fit that becomes trapped in a false local minimum. Incorrect timing means timing with an much larger error than the expected $\sigma$. These may be caused by an unresolved cycle ambiguity of the signal pulse in the cross correlation procedure. In order to reduce this, we compare the timing with it from another cross-correlation with different time window of signal waveform; $\left|t-t_{\text {peak }}\right| \leq 12 \mathrm{~ns}$, then reject the event if the difference is greater than $0.5 \mathrm{~ns}$ in any of $N_{\text {base }}$ baselines.

We also reduce the misreconstruction events using the excellent directivity characteristic of the ANITA horn antenna. Because of relatively high gain of the horn antennas, and corresponding narrow width of their angular response, the sector $\Phi_{\max }$ should be consistent with the signal direction. Therefore, we require $\left|\phi-\phi_{\max }\right|<-22.5^{\circ}$, where the $22.5^{\circ}$ is corresponding to the $\Phi$ occupancy of an antenna.

A further requirement for the misreconstruction reduction is an internal consistency check with sub-sets of the baselines. We perform angular fits not using one of the baselines in the original baseline set. We reject events if any of the $N_{\text {base }}-1$ of possible subset fits provides a space angle result that differs from the original by more than $5^{\circ}$.

\section{F. Efficiency}

The final reconstruction efficiency is important to understanding the overall detector efficiency, and is measured with the ground based calibration data and additional simulation analysis. Figure 30 shows the efficiency measurement as a function of $S N R$ for events acceptable fits for both polarizations. There is a good agreement between the ground based 

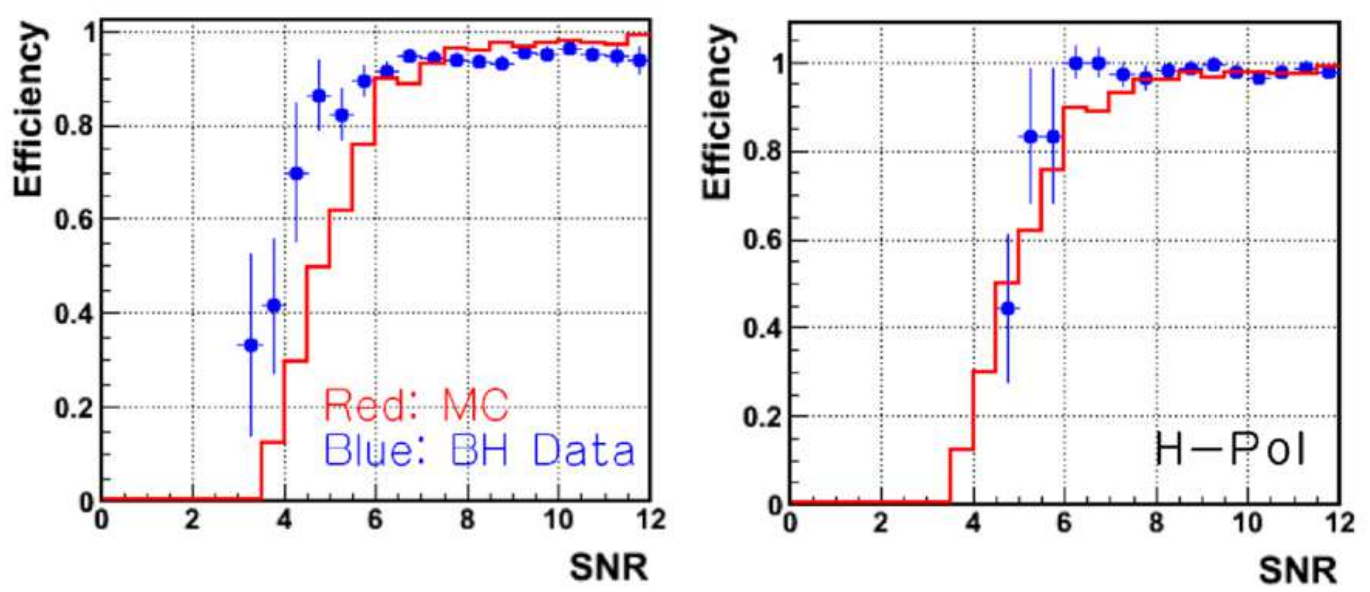

FIG. 30: Reconstruction Efficiency vs.SNR for both polarizations analyzed independently: Dots are efficiency measurement with the ground based calibration pulses and the curves is for instrument simulation Monte Carlo estimation. Left is for vertical polarization which has a known SNR bias due to low-level EMI contamination in the event signals. The right shows the same efficiency for H-pol which is less affected by EMI contamination.

calibration data and the MC in $S N R>5.5$ while a discrepancy is found in the lower $S N R$ region for the Vpol events. The likely source of this discrepancy is the effect of low-level interference from Williams Field where the ground calibration system was used, which causes an error in the SNR estimate. Due to the preponderance of verticallypolarized signals in the transmitters near Williams field, Vpol is affected to a greater degree than H-pol. The global efficiency at higher SNR for both polarizations with the ground calibration system is $96 \%$ while a misreconstructionreconstruction rate is less than $0.16 \%$.

\section{G. RF Projection on the Surface}

As noted above, reconstruction of the RF source position on the surface is critical to identify the anthropogenic noise sources associated with known bases. In order to obtain more precise position measurements we take into account the surface elevation variation based on the Bedmap data. [43] A simple model is implemented for a fast calculation. We find line-sphere intersections assuming the spherical shape of the earth, then take one solution having shorter distance to the ANITA payload. For an initial calculation, we assume the earth radius $R_{\text {earth }}^{\text {hypo }}$ is the geoid plus surface elevation of the ANITA payload coordinates (longitude and latitude). Then the next iteration uses the geoid and surface elevation of result coordinate of the previous iteration. We found the results converge after more than 2 iterations. We found the source position resolution at the surface is $2.7 \mathrm{~km}$ for $170 \mathrm{~km}$ of line-of-sight distance at $37 \mathrm{~km}$ of altitude.

\section{SUMMARY}

We have presented the basis for a balloon-borne payload search for ultra-high energy neutrinos from an altitude of $37 \mathrm{~km}$ above the Antarctic Ice sheets. The ANITA payload's first flight resulted in a large database of impulsive events from locations all across Antarctica. When these events indicated a ground-based point source, it could be geolocated to a precision of order $0.3^{\circ} \times 1.0^{\circ}$ in angle, which projects to error ellipses of several square $\mathrm{km}$ at typical distances of several hundred $\mathrm{km}$. We have demonstrated that operation down to near-thermal-noise levels is achievable, and electromagnetic interference in Antarctical, while still problematic, is manageable and largely confined to a relatively small number of camps. Results from the analyses of all these data in the search for neutrino candidates will be presented separately. 


\section{APPENDIX A: EXTENSIVE AIR SHOWER RADIO AND ANITA-1}

Radio Detection of extensive air showers (EAS) has recently received renewed attention as a method for detection and characterization of EAS. The radio emission is primarily produced by synchrotron effects in the geomagnetic field, and so has been termed geo-synchrotron emission [38]. This type of emission dominates over Askaryan emission from the charge excess since all of the charge, the sum of both positrons and electrons, contributes to it as the component trajectories curve in opposite directions in the magnetic field. The emission is highly beamed along the shower axis, with a characteristic beamwidth of a few degrees or less. It is linearly polarized, and coherent at frequencies below about $100 \mathrm{MHz}$, and partially coherent above $200 \mathrm{MHz}$, but these values vary widely with observation angles and the distance to the shower axis. The partially coherent emission has been detected up to hundreds of MHz [39, 40, 41], with some evidence for a flat radio spectrum above $200 \mathrm{MHz}$ [39]. Thus ANITA-1 may be able to detect such events both as direct emission when the shower axis points almost directly at the payload from above the horizon, or potentially as reflected events when the emission undergoes near-specular reflection off the ice surface.

In the latter case, such events could be a background for neutrino signals, if they are not distinct in some way. In fact, such events are likely to be highly horizontally polarized for two reasons: (1) They are intrinsically stronger in Hpol because the geomagnetic field is more vertical near the poles, and thus the field bending of the charges for moderately inclined showers (seen in reflection) yields mostly intrinsic Hpol; and (2) the Fresnel reflection coefficient for TE waves at the surface is always larger than for TM waves, and over most of ANITA's aperture, it is much larger. Thus even an unpolarized initial signal will acquire horizontal polarization, and Hpol will tend to be accentuated even more for signals with some intrinsic Hpol. Figure 31 shows the base-10 logarithm of ratio of reflected intensity for the TE vs TM wave. It is evident that it exceeds a factor of 10 over much of ANITA's angular range of acceptance.

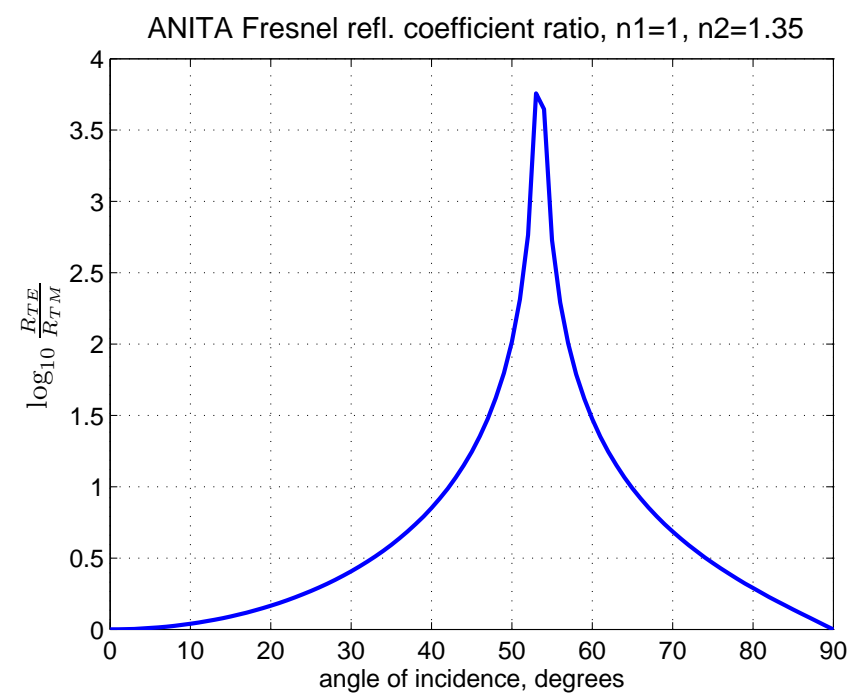

FIG. 31: Fresnel coefficient ratio for Te vs TM waves, resulting in H-polarization for most reflected signals.

\section{Simulation.}

To simulate the geosynchrotron radio emission for ANITA's band, we made use of a recent detailed simulation by Huege \& Falcke [27] (hereafter HF05) using CORSIKA for the EAS simulation, married to a standard electromagnetic radiation treatment for the radio emission. HF05 were mainly interested in the lower frequency bands below $100 \mathrm{MHz}$, but they did extend their simulations to the partially coherent region up to about $500 \mathrm{MHz}$, although none of their parameterizations are strictly valid there as they were only fit for the coherent regime. But we use the reported field strengths in the 200-500 MHz regime to create an ad hoc parameterization. Based on the given results, the emission is nearly flat with frequency above $200 \mathrm{MHz}$ in all cases, but varies widely with the distance away from the shower axis. Energy scaling is not reported but can be heuristically estimated from the reported results as well.

Because the average sine of the magnetic field inclination angle is close to 1 for all of Antarctica [31], and since 
ANITA's antenna geometry favors showers inclined by $45^{\circ}$ or more, we treat all showers as producing predominantly horizontally polarized signals as seen in ANITA's projection; this is evidently true on average. The vertically polarized component on average is less than $1 / 3$ of the Hpol in field strength, and after reflection this ratio is increased even more. As a result, detection of air showers in the Vertically polarized component is strongly suppressed, and we will quantify to what level in a later update.

With these assumptions, we use

$$
\mathcal{E}=10^{-5}\left(\frac{E_{\text {shower }}}{10^{19} \mathrm{eV}}\right)^{0.85} \frac{R_{0}}{R} e^{-\theta / \theta_{0}} \rho\langle\sigma\rangle \beta \zeta \xi \text { Volts m }^{-1} \mathrm{MHz}^{-1}
$$

where $\mathcal{E}$ is the field strength at the payload; $E_{\text {shower }}$ is the shower energy in $\mathrm{eV} ; R_{0}=6.7 \mathrm{~km}$ is a reference distance related to the effective origin of the shower radio emission above the ground; $R$ is the distance from the point of reflection to the payload; $\theta$ and $\theta_{0}=5.1^{\circ}$ are the angle with respect to projected shower axis, and a characteristic angle based on the radial distances of exponential falloff with respect to the shower axis; $\rho$ is the field reflection Fresnel coefficient; $\langle\sigma\rangle=0.95$ is the average sine of the magnetic field inclination with respect to the horizontal, and $\langle\sigma\rangle$ here thus gives the first-order average fractional component of the horizontally polarized field strength; $\beta$ is the ratio of the Antarctic mean magnetic field strength to the B-field strength used in the HF05 calculations $(\beta=1.08$ in our case); $\zeta$ is a factor to account for the fact that the HF05 estimates are in the radio near-field; and $\xi$ is a factor to account for surface roughness scattering losses.

The value of $\theta_{0}=5.1^{\circ}$ is about a factor of two higher than estimates for vertical showers at ground level. HF05's results for inclined showers do however indicate that showers at $45^{\circ}$ or more have much flatter exponential falloff than vertical showers, even after correcting for projection effects, and the value adopted here is consistent with the simulation data presented in HF05.

For the present results we are using $\zeta=1.2$ as indicated by HF05's scaling with inclined showers, which tend to move the observer out of the near field. In general, near field effects reduce the relative field strength as compared to the far-field, so $\zeta>1$ is to be expected. We have also assumed $\xi=0.9$ in reflection-since the average wavelengths are long and the refractive index contrast low, we expect surface roughness to play a minor role here. We do not include surface slope variations here; the surface is assumed to be the locally flat portion of a sphere. We have assumed flat emission with frequency based on the reported HF05 results up to $500 \mathrm{MHz}$ and weakly supported by earlier observations [39]; above this we have arbitrarily reduced the field strength by factors of 0.7 and 0.5 for the MID2 and HIGH bands respectively.

Events are simulated over the entire ANITA field of view (assuming phi symmetry), and several adjacent phi-sectors are used to form a trigger. A standard 3 of $8 \mathrm{~L} 1$ trigger using LCP+RCP is used, with proper Rician noise and signal estimation. The antenna beam patterns are included. The EAS signal is assumed to be initially completely Hpol. The exponential off-axis attenuation determined by $\theta_{0}=\tan ^{-1}(d / D)$ where $d$ is the off-axis characteristic distance for 1/e attenuation, and $D \sim 6.7 \mathrm{~km}$ is the distance to the shower effective origin. The value used in the near-field parameterizations is very conservative since it is based on vertical showers, where $d=300 \mathrm{~m}$ was indicated. The simulations for inclined showers indicate a value of $d=600 \mathrm{~m}$, and we have used this for the far-field ANITA case. Also, these values are energy dependent, but the dependence is currently difficult to estimate from the reported results so we have neglected it.

For the UHECR shower rates and absolute normalization, we used the Auger 2007 normalization and the Auger 2007 parameterization of the empirical GZK cutoff as observed in the Auger data. The integral flux above $10^{19} \mathrm{eV}$ is $J(>E)=2.0 \times 10^{-18} \mathrm{~cm}^{-2} \mathrm{~s}^{-1} \mathrm{sr}^{-1}$. This spectrum was sampled via a von Neumann method.

\section{Results.}

The simulation predicts a total of $4.5 \pm 0.4$ (statistical) detected events for 18 days of cumulative livetime for the ANITA-1 flight. However, the systematic errors on this result are estimated to be of order $100 \%$-that is, our result is also consistent with zero detected events. This level of uncertainty stems from the high degree of uncertainty on simulation of the partially coherent signals from EAS in the ANITA band, which is well above the region where EAS radio measurements are primarily focused.

Putting aside the issue of the absolute detected flux, we may still investigate the relative energy spectral content of the detected events. Figure 32 shows the energy distribution of the detected events; here once again the overall flux levels are subject to large systematic, although the relative fluxes are less sensitive to these same systematics. The energy threshold is about $4 \times 10^{19} \mathrm{eV}$ and shapes the low-energy edge of the distribution. The GZK cutoff itself shapes the high energy edge. 

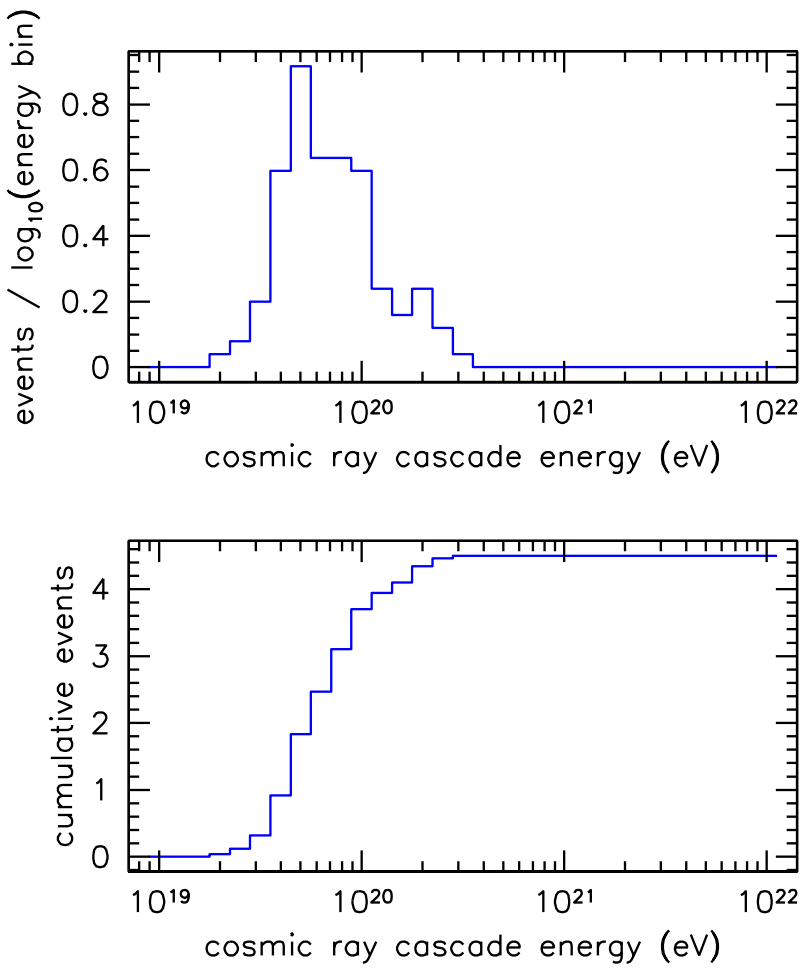

FIG. 32: Top: Differential energy distribution of simulated events that are detected by ANITA-1. Bottom: Cumulative energy distribution of events, showing that the detectability turns on in the vicinity of the GZK cutoff.

In summary, ANITA's sensitivity was adequate to possibly detect a handful of events from extensive air shower radio emission, seen in reflection from the ice surface. The uncertainties in this prediction are dominated by the uncertainties in the magnitude of partially coherent emission from EAS radio processes.

\section{APPENDIX B: CHARACTERIZATION OF SURFACE ROUGHNESS AND ITS EFFECT ON NEUTRINO APERTURE}

One concern for modeling the detection of neutrinos in the ice is the propagation of the radio emissions from under the ice to the surface, i.e., the firn, into the air. If the surface of the ice were a smooth boundary, we could simply apply the proper Fresnel coefficient for each polarization at the surface corresponding to an index of refraction $n \simeq 1.3$ to air $n=1$. However, the Antarctic winds and other effects serve to carve up the surface, making it rough on many length scales.

RF energy propagating from the neutrino interaction to the instrument does not pass through a single point on the surface of the ice. Rather, all radiation passing through an area given by the first Fresnel zone on the ice surface can contribute to the received band-limited impulse. This region is determined by considering the locus of points on the surface where the pathlength is within a quarter-wavelength of the direct geometric ray for an idealized smooth surface. Straightforward geometric considerations for a simple phase-screen perpendicular to the direction of propagation between a transmitter and receiver give a first Fresnel zone radius

$$
R_{F, 1}=\sqrt{\frac{c d_{1} d_{2}}{\langle f\rangle\left(d_{1}+d_{2}\right)}}
$$

where $c$ is the velocity of light, $\langle f\rangle$ the mean frequency of the waves, and $d_{1}, d_{2}$ are the source-to-phase screen and receiver-to-phase screen distances, respectively. Within this radius, any sub-wavelength phase disturbance to the propagating waves will cause constructive or destructive interference to the beam. Higher order Fresnel zones at larger radii may be important in some cases, but we neglect them here. 


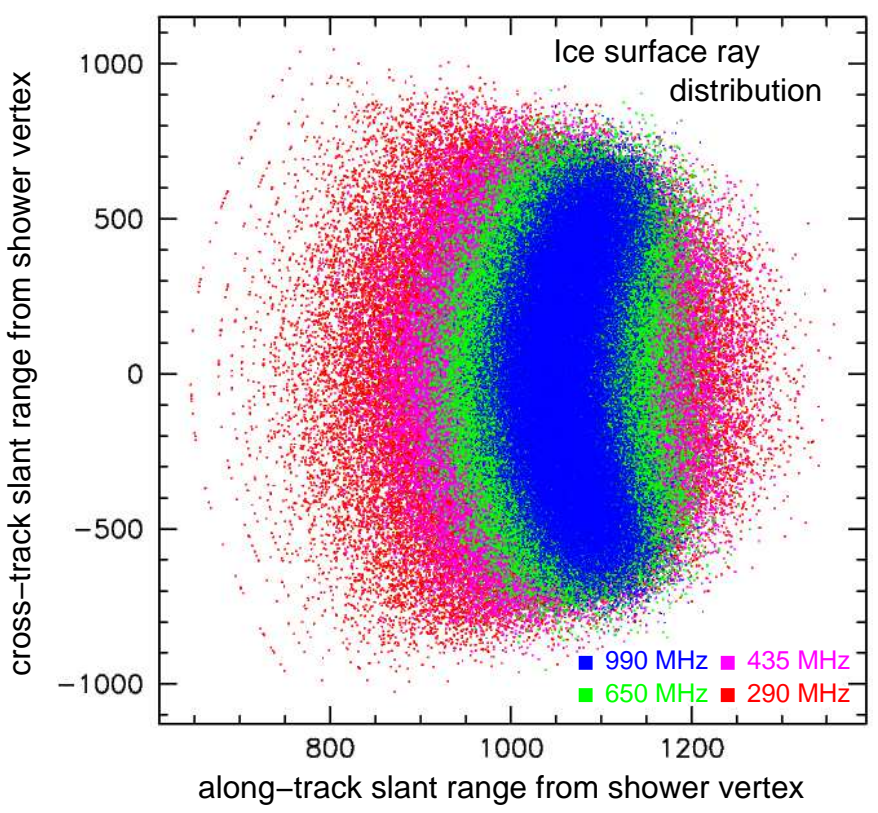

FIG. 33: The distribution of ray exit points on the ice surface from a near-horizontal neutrino event at a depth of about $1 \mathrm{~km}$. The colors indicate the ray patterns for the center frequencies shown.

In ANITA's case, the Fresnel zone geometry must be modified to account for the intervening medium between the neutrino shower "transmitter" and the payload receivers. In addition, projection effects must be considered for the oblique incident angles of the Cherenkov radiation on the underside of the ice surface, and the often steep refracted angles of near-tangential outgoing rays. Accounting for these effects, and the Snell's law divergence associated with them, the modified first Fresnel zone for ANITA is an approximate ellipse with a major axis diameter, transverse to the plane of incidence and reflection along the ice surface, of

$$
D_{F}^{t r}=2 \sqrt{\frac{c}{\langle f\rangle} \frac{d_{2}}{1+n \frac{d_{2}}{d_{1}}}}
$$

and a minor axis along-track diameter of

$$
D_{F}^{\ell}=\frac{2}{\sin \theta_{i}} \sqrt{\frac{c}{\langle f\rangle} \frac{d_{2}}{1+\mu \frac{d_{2}}{d_{1}}}}
$$

where $\mu=n\left(\cos \theta_{i} / \cos \theta_{r}\right)^{2}$ and $\theta_{i}, \theta_{r}$ are the incident and refracted angles with respect to the normal for the underside of the ice surface.

Thus, for example, for a mean frequency of $300 \mathrm{MHz}$, an index of refraction of $n=1.35$ corresponding to the near-surface firn, a near-tangential refracted angle of $85^{\circ}$, an incident angle on the underside of the ice surface of $47.5^{\circ}$, and distances $d_{1}=1400 \mathrm{~m}$, and $d_{2}=300 \mathrm{~km}, D_{F}^{t r} \simeq 50 \mathrm{~m}$ and $D_{F}^{\ell} \simeq 11 \mathrm{~m}$, many wavelengths across in either dimension. Figure 33 shows a simulation result for radio Cherenkov emission from a shower similar to this example. Here only rays that can escape total-internal-reflectance are shown, and the colors show the ray patters for different frequencies. It is evident that the scale of the emission at the surface for a typical shower is much larger than the first Fresnel zone for any of the frequencies of interest to ANITA; thus in almost all cases the Fresnel zone scale is the relevant scale for surface roughness effects.

Variations in the surface over the Fresnel zone scale potentially alter the transmission relative to a smooth surface. Overall surface tilts on scales larger than this size are of order 1 degree and serve only to tip the refracting surface slightly; the Fresnel coefficient for a plane wave still applies. To this end, a distribution of slopes at random angles is employed in the simulations. Since most of our events are close to total-internal-reflection for a horizontal surface, this variation in slope tends to increase ANITA's overall aperture. We distinguish this effect from the optically more complicated effect of roughness on scales within the first Fresnel zone. 
Surface "facets" on scales of order a wavelength or less will affect the tranmission properties, and they must be integrated over the scale of the Fresnel zone to assess their net effect. In the firn ANITA's wavelengths are 0.2 to $1.2 \mathrm{~m}$. As described below, the Antarctic surface has roughness on scales smaller, equal, and larger than this range. We are presented with a problem similar to a frosted glass effect in the optical case where irregularities on the scale of a wavelength can significantly disturb the angular distribution of transmission [53]. In principle the effect could either increase or decrease ANITA's sensitivity. The spreading of the radio Cherenkov cone will serve to increase (worsen) ANITA's energy threshold. However, the spreading of the cone could also greatly improve the solid angle for neutrino detection by allowing ANITA to see events that would have been totally internally reflected by a smooth surface.

\section{Antarctic Surface Roughness Data}

Quantitative studies of the Antarctic surface roughness comes from two sources: Over-snow traverses [54, 55] have characterized surface features in detail, but only in limited areas, mostly in East Antarctica or near the coasts. Satellite altimeter data covers about $80 \%$ of the continent but with limited resolution and scales on the order we care about are difficult to extract. Over-snow traverses identify roughly three types of features. Depositional surface features or "snow dunes" are typically 50-100 m long, 10 - 15 wide and $1 \mathrm{~m}$ high. Redistribution surfaces are usually $20-50 \mathrm{~m}$ long, $2-3 \mathrm{~m}$ wide and $0.3-0.5 \mathrm{~m}$ high. Erosional surfaces are $0.3 \mathrm{~m}$ in mean height. The term sastrugi in the literature can refer either to erosional surfaces exclusively, or to any meter-scale feature. Features larger than 1 meter tend to align along the direction of the prevailing winds. Features on the centimeter scale are micro-roughness.

The reader may have a mental picture of the Antarctic surface as covered with large sastrugi, a picture perhaps biased by the most interesting photography of the continent. However, in reality, such features are rare. The oversnow traverse of Furukawa [54] on the East Antarctic plateau (along a $1000 \mathrm{~km}$ traverse at $40^{\circ} \mathrm{E}$ from $69^{\circ} \mathrm{S}$ to $77^{\circ} \mathrm{S}$ ) encountered features larger than 0.3 meters at most 75 times per kilometer ( 1 per 13 meters) and typically 50 times per kilometer (1 per 20 meters), i.e., 5\% of the surface. The traverse of Goodwin [55] provides complementary data that shows the average feature size only exceeded 0.5 meters during $7 \%$ of their 750 kilometer traverse (traverse at $72^{\circ} \mathrm{S}$ latitude from $112^{\circ}$ to $132^{\circ} \mathrm{E}$ ). Only once on their $750 \mathrm{~km}$ journey do they report sastrugi that are 2 meters high. Hence we may extract that the fraction of the area of the East Antarctic plateau covered by even half-meter features to be less than $5 \%$ of the surface. Although we do not have traverse data from West Antarctica, anecdotal evidence from skiers travelling from Patriot Hills to South Pole indicates that these features are rare there as well. The risetime of pulses transmitted by satellite altimeters (Seasat, ENVISAT, ERS), which is sensitive to the size of surface features, shows the western plateau to be only about $25 \%$ rougher than the eastern plateau.

A compilation of the traverse and altimeter data can be found in Ref. [56]. We summarize the data here as containing features with $30 \mathrm{~cm}$ typical heights and $8 \mathrm{~m}$ spacing or $70 \mathrm{~cm}$ typical heights and $13 \mathrm{~m}$ spacing. Hence the features have vertical scales comparable to a wavelength with separations of many wavelengths. Considered as angles, these roughness features would be of order 2 degrees.

Members of the ANITA group made quantitative measurements of roughness features at one location on the Antarctic Plateau, near Taylor Dome [57]. Hundreds of measurements of angles on a $0.5 \mathrm{~m}$ scale with 0.1 degree precision are shown in Figure 34. The RMS distribution of angles on the $0.5 \mathrm{~m}$ scale had an RMS of 1.7 degrees, consistent with the traverse data above. The ordered angle measurements yield an elevation map, shown in Figure 35 . We were concerned with correlations between angles in orthoganal directions along a traverse. As shown in Figure 36 , data taken in a 2-dimensional surface grid show essentially no correlation of angles between measurements taken in perpendicular directions. We made larger scale $(100 \mathrm{~m})$ measurements at a few points and angles of order 0.3 to 1.1 degrees were measured, again consistent with the traverse data. An overall slope of the area on the scale of 1 degree was measured, consistent with the known glacier flow.

\section{Optical Modeling}

The scale of roughness with horizontal scales of several wavelengths and vertical scales of order a wavelength is a difficult region to model mathematically since few electromagnetic approximations are valid in this regime. We emulated the situation experimentally and scaled to the optical regime [58]. The radio transmission was modeled by a $632 \mathrm{~nm}$ laser and incoherent red light source. The surface roughness was modeled by a range of diffusers: 400-, 1000-, and 1500-grit diffusers. We measured the surface features of the diffuser with a Veeco atomic force microscope and found the characteristics of the 1500 grit diffuser $(200-500 \mathrm{~nm}$ height, $400-4000 \mathrm{~nm}$ transverse feature size) and $632 \mathrm{~nm}$ light to closely emulate the Antarctic surface for the decimeter radiation ANITA detects. 

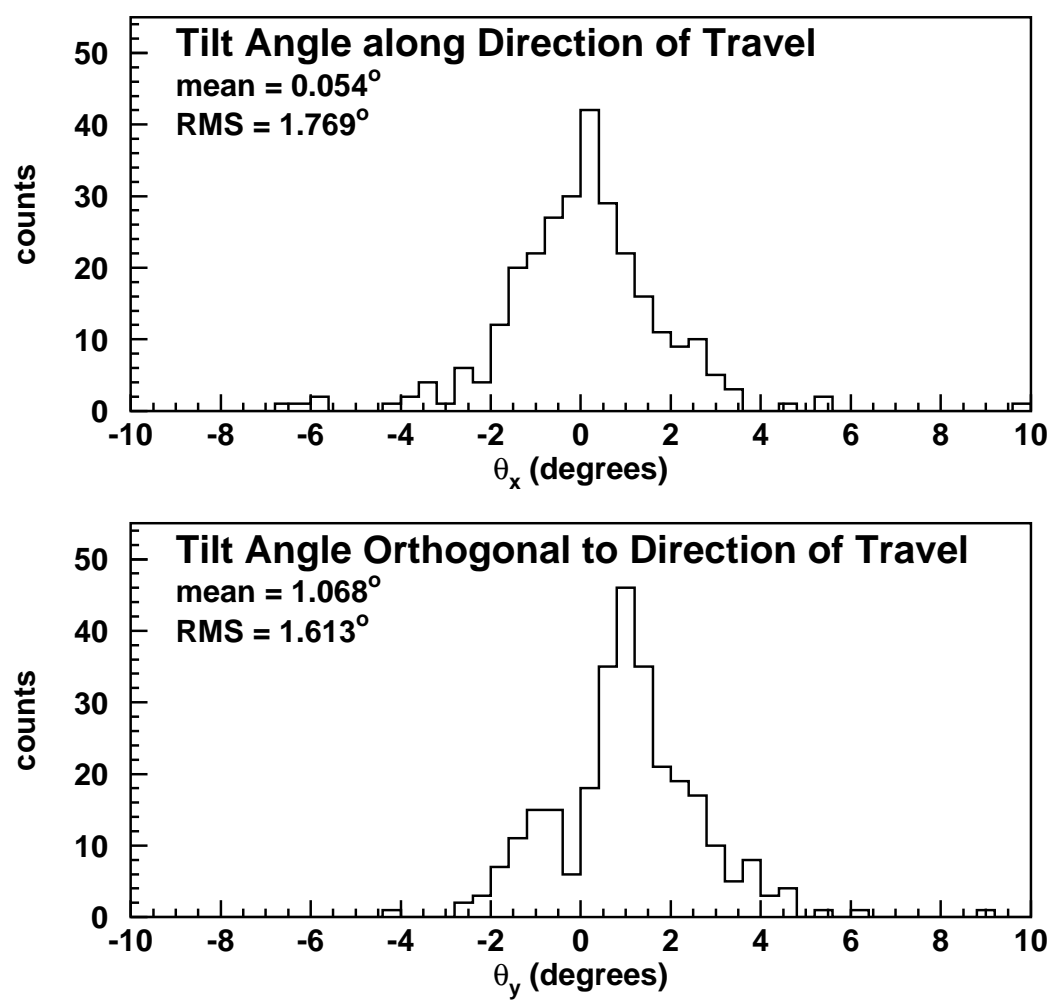

FIG. 34: Left: distribution of angles measured by placing a $0.5 \mathrm{~m}$ plate on the surface of the snow at Taylor Dome. Angles were measured sequentially along a $100 \mathrm{~m}$ traverse in the prevailing wind direction. Right: Orthogonal angle measured along the traverse.

We affixed a half cylinder to the back of the diffuser so that the incident light would always enter the glass perpendicularly and not be refracted on its way into the glass. In this way, all angles could be probed, especially beyond that of total-internal reflection if the surface were smooth. The coherent and incoherent light sources gave the same results. We observed, as expected, that there is a wider distribution of scattered light, but this was still relatively small compared to the natural width of the Cherenkov cone from the Askarayan effect. The total integrated power within a few degrees of the specular result stayed the same so radiation is not lost, only spread. We also observed significant radiation beyond the total internal reflection angle. To our knowledge, this experiment was the first to observe the predicted [59] suppression of Snell's Law relative to the specular case so that rays are less bent than a $n=1.5$ to $n=1$ transition. The effect increased with roughness, as expected, and deviations as large as 20 degrees were observed.

The simulation employs a simple "facet model" [59] of roughness, performing ray tracing through small smooth faces on scales smaller than a wavelength. We saw in the optical case, this simple geometric model reproduces the data extremely well [60]. We input the measured atomic force microscope (AFM) surface data from the same diffusers we used into the electromagnetics simulation code Advanced Simulation Analysis Program (ASAP), developed by the Breault Corporation for simulation of optical systems. To test the facet model, we ran the code with diffractive effects turned off. All the angular distributions, including intensity and the suppression effect of Snell's law could were reproduced to high accuracy using the ASAP simulation. A typical plot of data versus simulation is shown in Figure 37. The vertical bar shows the refraction angle expected by Snell's law, which is suppressed for the rough surface. Even using only geometric optics, ASAP successfully reproduces the widths of the intensity distributions and the observed Snell's Law suppression. (The shoulders in the simulation are due to finite statistics of the surface roughness model employed.) The observed transmission beyond total-internal reflection (TIR) angle was also measured and simulated. For example, the intensity at an angle beyond TIR (70 degrees) was measured to be about $17 \%$ of the peak transmission, while the simulation yielded $20 \%$, demonstrating good agreement. The success of the facet model to reproduce the 


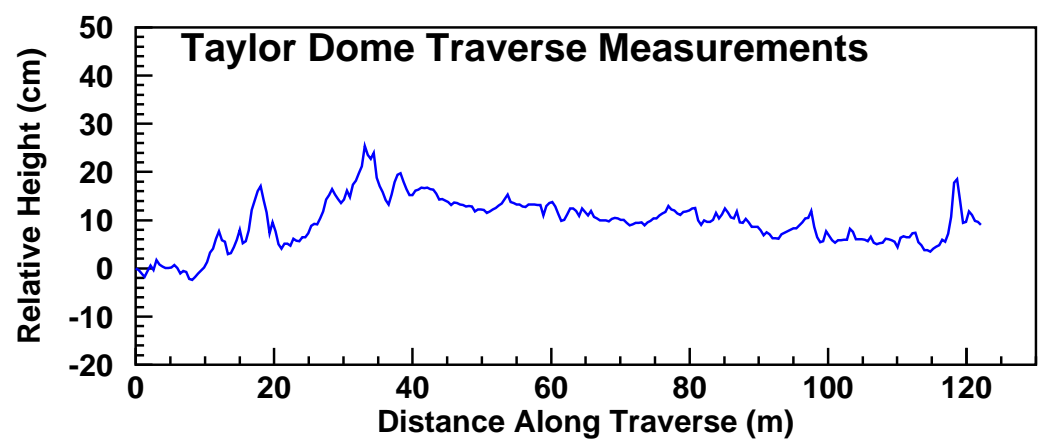

FIG. 35: Elevation map created by integrating the measured angles over the length of the 100 m traverse at Taylor Dome.

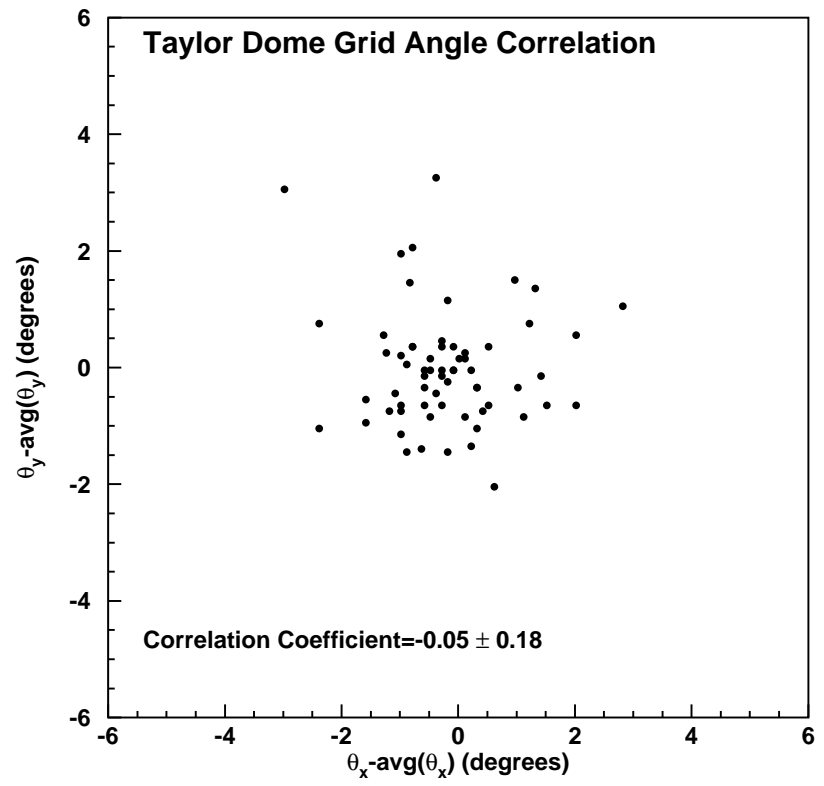

FIG. 36: Scatter plot of angles measured in a grid taken with the $0.5 \mathrm{~m}$ scale at Taylor Dome. No significant correlation between orthogonal directions was observed.

optical data gives us confidence that the Monte Carlo simulations which use the same facet model are essentially correct.

The micro-roughness scale is much less than one-tenth of a wavelength and is ubiquitous. The effect is difficult to quantify either for Antarctica or for the ground glass diffusers. However, by their construction, sandblasting of glass, the diffusers surely do have microscale roughness as well, but the ASAP simulation described the data well without including the effect. So the same is likely true for Antarctica and the decimeter radiation.

In addition to the optical models we performed a laboratory test in the radio-frequency domain as well. During our run using an ice target at the SLAC linear accelerator [21], we took several runs where we placed rows of ice $20 \mathrm{~cm}$ high separated by $50 \mathrm{~cm}$ intervals across the surface of the ice target. This was a deliberate overestimate of the antarctic roughness extracted from traverse and satellite data to look for some effect. We observed an on-axis reduction of recorded voltage of $17 \%$. Off-axis, the effect dropped to $8 \%$. We may infer the effect of roughness on 
GRIT 400.50, $40^{\circ}$ incidence, $n=1.5$

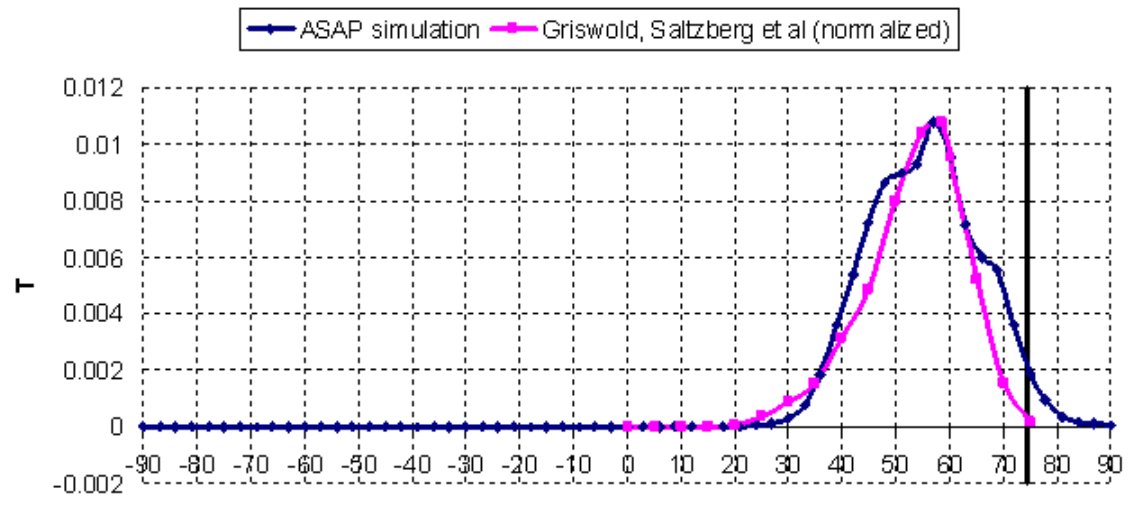

FIG. 37: A representative comparison of the optical diffuser intensity data vs the ASAP simulation. Pink is data and blue is simulation. Details in the text.

ANITA is minimal, hurting the threshold by less than of order $10 \%$.

\section{Time-domain concerns}

The optics test used a continuous-wave source rather than an impulse. It is possible that the emissions are spread out in time as well. Since the pathlengths through the rough surface, even with multipath, are an order of magnitude smaller than our trigger integration time of $7 \mathrm{~ns}$, we do not expect an effect. To search for the effect in data, we used the in-ice disccone placed at $97 \mathrm{~m}$ depth at Taylor Dome during the ANITA flight. At a distance of $200 \mathrm{~km}$, we reconstructed approximately 20 events. As shown in Figure 25 in the main text, no time spreading is observed. When deconvolved with the known instrument response, the signal is consistent with a delta function indicating time domain spreading effects, if there, are much smaller than the sample interval of $400 \mathrm{ps}$ and do not effect the neutrino detection. For ANITA-II we plan to measure this effect right down to the horizon, where the effect may be larger, but most of ANITA events come from a distance already tested here.

\section{Implementation into Simulations}

The UH ANITA simulation is based on multiple ray tracing and could most directly incorporate both the deleterious effect of beam spreading and advantageous effect of being able to see beyond the total-internal reflection (TIR) angle. The net result is that the extended viewing beyond TIR dominates the event rate and boosts the high energy neutrino acceptance by $50 \%$. At lower energies events are lost due to raising the detection threshold. Overall acceptance over a GZK spectrum is affected by about $10 \%$ so the corresponding uncertainty on the neutrino flux limits, after including the effects of surface roughness to first order, are not significant.

\section{APPENDIX C: THERMAL-NOISE ACCIDENTAL RATES FOR ANITA}

\section{The single-antenna trigger}

At the single antenna level (Level 1, or L1), we require three-of-eight of the dual-circular-polarization, fourfrequency-sub-bands to trigger in hardware. Each of these bands involves a coincidence window with an effective time width of $T_{1}=19 \mathrm{~ns}$. However, if the trigger is to survive all software cuts, it must also be coherent across all of the sub-bands, that is, it must be in phase, a much tighter requirement than the hardware trigger.

For thermal noise, the phases are completely uncorrelated, and the probability for thermal noise to produce a coherent impulse in each sub-band which is also coherent across the bands is just given by the ratio of the coherence time 
$\tau_{c o h}$ of the full-band impulse to the total coincidence window, multiplied by the number of independent trials for a coherent signal within the coincidence window to produce the 3 of 8 trigger combination. The number of combinations of $N$ subbands taken $M$ at a time gives a combinatoric factor of $C=N ! /(M !(N-M !))$, and for $N=8, M=3$ we have $C=56$. For any given combination of $M$ sub-bands that gives an L1 trigger, there are

$$
\left(\frac{T_{1}}{\tau_{c o h}}\right)^{M}
$$

combinations of sub-band signals that can trigger, but only those for which the relative locations in the sub-bands are identical (ignoring constant offsets for sub-band differential delays) can yield a coherent full-band pulse. There are thus $T_{1} / \tau_{c o h}$ such coherent combinations. The probability of obtaining one such combination is thus

$$
P_{1}=C\left(\frac{\tau_{c o h}}{T_{1}}\right)^{M-1}
$$

The coherence time $\tau_{c o h}$ is estimated by requiring that the signal be phase-coherent across the full-band, and thus that the sub-bands have less than a $\lambda / 8$ phase offset between them for a wavelength at the mean frequency $\bar{f}$ of the band. For $\bar{f} \simeq 600 \mathrm{MHz}$ as for ANITA, the delay corresponding to a $1 / 8$-wave phase difference is $\tau_{\text {coh }} \simeq 200 \mathrm{ps,} \mathrm{which}$ gives $P_{1}=6.2 \times 10^{-3}$.

\section{The level 2 and 3 trigger: azimuth+elevation constraint}

For the L2 trigger, adjacent antennas must each have an L1 trigger with 5 ns of overlap for a $30 \mathrm{~ns}$ L1 one-shot output, giving an effective coincidence window of about $T_{2} \simeq 40 \mathrm{~ns}$. The number of possible combinations of pairs of L1 impulse arrival times that can produce L2 triggers is $T_{2} / \tau_{a z}$ where $\tau_{a z}$ is the azimuthal time resolution for pair of antennas within an azimuthal ring. Similar analysis applies to the elevation constraint via the L3 trigger, which couples the upper and lower rings. The coincidence window for L3 triggers is the convolution of the L2 windows, and gives $T_{3} \simeq 50 \mathrm{~ns}$.

However, the analysis requires an additional azimuth constraint in the other ring, combined with a pair of elevation constraints for adjacent phi-sector pairs. To estimate the fraction of such combinations of 4-antenna triggers that add coherently to give a proper direction reconstruction, we take the ratio of the geometric mean of the elevation and azimuth time resolutions to the total number of combinations of random impulses that can form in the $4 T_{2}$ coincidence windows required for an L3. This represents the probability that a single direction (the normal incidence plane wave) can be mimicked by thermal noise:

$$
p_{2}=\left(\frac{\sqrt{\tau_{a z} \tau_{e l}}}{T_{2}}\right)^{4}
$$

Multiplying this by the number of independent directions within the antenna beam, and by the number of phi sectors $N_{\phi}$ then gives the total probability at the L3 level.

For a beam with $\Omega=\pi \Delta \theta \Delta \phi \simeq \pi\left(0.3^{\circ}\right)\left(1.0^{\circ}\right)=0.94$ square degrees, and an antenna main beam solid angle of $\Omega_{\text {ant }} \simeq \pi \Phi^{2} / 4$ for a half-power beam width $\Phi \simeq 40^{\circ}$, we finally get

$$
P_{2}=N_{\phi} K \frac{\Omega_{a n t}}{\Omega} p_{2}
$$

where $K$ is the fraction of the main antenna beam that is allowed (eg, the fraction below the horizon). For ANITA, the geometric mean of the timing errors is about $60 \mathrm{ps,} \mathrm{and} \mathrm{thus} P_{2} \simeq 5.4 \times 10^{-8}$, for $K=1$.

\section{Combined probability}

Combining the L1 probability and the L2 probability since they are independent for thermal noise, the total probability for a thermal noise trigger to produce an acceptable direction reconstruction is

$$
P_{\text {tot }}=P_{1} P_{2}=\left(6.2 \times 10^{-3}\right) \times\left(5.4 \times 10^{-8}\right)=3.4 \times 10^{-10}
$$


or about 0.003 events expected during ANITA's flight, based on 8.2M triggers.

[1] K. Greisen, Phys. Rev. Lett. 16, 748 (1966); G. T. Zatsepin and V. A. Kuzmin, JETP Lett. 4, 78 (1966) [Pisma Zh. Eksp. Teor. Fiz. 4, 114 (1966)].

[2] V. S. Beresinsky and G. T. Zatsepin, Phys. Lett. B 28, 423 (1969).

[3] G. A. Askaryan, 1962, JETP 14, 441; 1965, JETP 21, 658.

[4] P. W. Gorham, C. L. Hebert, K. M. Liewer, C. J. Naudet, D. Saltzberg, D. Williams, Phys. Rev. Lett. 93 (2004) 041101.

[5] I. Kravchenko et al., Astropart.Phys. 20 195-213 (2003).

[6] P. W. Gorham, D. Saltzberg, R. C. Field, et al., Phys. Rev. D 72, 023002 (2005).

[7] J. V. Jelley et al., Nature 205, 327 (1965); Nuovo Cimento A46, 649 (1966); N. A. Porter et al., Phys. Lett. 19, 415 (1965); S. N. Vernov et al., Pis'ma v ZhETF 5, 157 (1967) [Sov. Phys.-JETP Letters 5, 126 (1967)]; Can. J. Phys. 46, S241 (1968); P. R. Barker, W. E. Hazen, and A. Z. Hendel, Phys. Rev. Lett. 18, 51 (1967); W. E. Hazen, et al., ibid. 22, 35 (1969); 24, 476 (1970); D. Fegan \& N. A. Porter, Nature, 1968; H. R. Allan, in Progress in Elementary Particles and Cosmic Ray Physics, v. 10, edited by J. G. Wilson and S. G. Wouthuysen (North-Holland, Amsterdam, 1971), p. 171, and references therein.

[8] The IceCube Collaboration: M. Ackermann et al., Nucl. Instrum. Meth. A556 (2006) 169.

[9] V. S. Beresinsky and G. T. Zatsepin, Phys. Lett. B 28, 423 (1969); R. Engel, D. Seckel and T. Stanev, Phys. Rev. D 64, 093010 (2001).

[10] G. A. Gusev, I. M. Zheleznykh, "On the possibility of detection of neutrinos and muons on the basis of radio radiation of cascades in natural dielectric media (antarctic ice sheet and so forth)," SOV PHYS USPEKHI, 1984, 27 (7), $550-552$.

[11] M. A. Markov, I. M. Zheleznykh, Nucl. Instr. Meth. A 248 (1986) 242.

[12] I. M. Zheleznykh, 1988, Proc. Neutrino 88, 528.

[13] R. D. Dagkesamanskii, \& I. M. Zheleznyk, 1989, JETP 50, 233.

[14] E. Zas, F. Halzen, \& T. Stanev, 1992, Phys Rev D 45, 362.

[15] J. Alvarez-Muñiz \& E. Zas, 1997, Phys. Lett. B, 411, 218.

[16] D. Saltzberg, P. Gorham, D. Walz, et al. Phys. Rev. Lett., 86, 2802 (2001).

[17] G. M. Frichter, J. P. Ralston, D. W. McKay, Phys. Rev. D 53 (1996) 1684-1698.

[51] J. Alvarez-Muniz, R.A. Vazquez and E. Zas, "Calculation Methods for Radio Pulses from High Energy Showers," astro$\mathrm{ph} / 0003315$.

[19] “Coherent Radio Pulses From GEANT Generated Electromagnetic Showers In Ice,” S. Razzaque, S. Seunarine, D. Z. Besson, D. W. McKay, J. P. Ralston, D. Seckel, Phys.Rev. D65 (2002) 103002; arXiv:astro-ph/0112505

[20] P. Miočinović, R. C. Field, P. W. Gorham, et al., Phys. Rev. D 74, 043002 (2006).

[21] ANITA Collaboration: P. W. Gorham, et al., Phys. Rev. Lett. 99 (2007), 171101.

[22] A. R. Jacobson, S. O. Knox, R. Franz, \& D. C. Enemark, "FORTE observations of lightning radio-frequency signatures: Capabilities and basic results," Radio Science 34, 337, (1999); D. C. Enemark, \& M. E. Shipley, "The FORTE receiver and sub-band triggering unit," Eighth Ann. Am. Inst. of Aeronautics and Astronautics, Utah State Univ. Conf. on Small Satellites, Logan, UT, 1994; Much information on FORTE can be gathered from http://forte.lanl.gov

[23] N. Lehtinen, P. Gorham, A. Jacobson, \& R. Roussel-Duṕre, Phys. Rev. D 69 (2004) 013008; astro-ph/030965.

[24] The ANITA Collaboration: S. W. Barwick et al., Phys. Rev. Lett. 96 (2006) 171101.

[25] A.R. Jacobson, pers. communication, (2001).

[26] A. R. Jacobson, K. L. Cummins, M. Carter, et al., "FORTE radio-frequency observations of lightning strokes detected by the National Lightning Detection Network," JGR 105, 15, 653 (2000); R. Roussel-Dupré, \& A. V. Gurevich, "On runaway breakdown and upward propagating discharges," JGR 101, 2297 (1996); A. V. Gurevich, K.P. Zybin, and R. Roussel-Dupré, "Lightning Initiation by simultaneous effect of runaway breakdown and cosmic ray showers," submitted to JGR (2001).

[27] T. Huege and H. Falcke, Astropart.Phys. 24 (2005) 116-136; astro-ph/0501580.

[28] D. Fegan \& N. A. Porter, Nature, 1968.

[29] S. Barwick, D. Besson, P. Gorham, D. Saltzberg, J. Glaciol. 51 (2005) 231.

[30] http://msp.gsfc.nasa.gov/tdrss/oview.html

[31] NOAA NGDC: http://www.ngdc.noaa.gov/geomagmodels/struts/calcGridIGRF

[32] G.S. Varner, "The Modern FPGA as Discriminator, TDC and ADC", Journal of Instrumentation, Vol. 1, P07001 (2006).

[33] R. Engel, D. Seckel, T. Stanev, "Neutrinos from Propagation of Ultrahigh Energy Protons, Phys.Rev. D64:093010

[34] S. Kleinfelder, "GHz Waveform Sampling and Digitization Circuit Design and Implementation”, IEEE Trans. Nucl. Sci. 50, No. 4, pp. 955-962, August 2003.

[35] S. Ritt, “The DRS chip: Cheap waveform digitizing in the GHz range.”, Nucl. Instrm. Meth. A 518:470-471 (2004).

[36] G. Varner et al., "Monolithic Multi-channel GSa/s Transient Waveform Recorder for Measuring Radio Emissions from High Energy Particle Cascades", Proc. SPIE Int. Soc. Opt. Eng. 4858-31, 2003.

[37] G.S. Varner, L.L. Ruckman, R.J. Nichol, J. Nam, J. Cao, M. Wilcox and P. Gorham, "Large Analog Bandwidth Recorder and Digitizer with Ordered Readout (LABRADOR) ASIC,” Nucl. Instr. Meth. A483:447-460 (2007). 
[38] H. Falcke \& P. Gorham 2003, Astropart.Phys. 19 (2003) 477-494.

[39] R. E. Spencer, "Radio Pulses from Cosmic Ray Air Showers at 44, 105, 239, and 408 MHz," Nature 222, 460 (1969).

[40] D. J. Fegan and P. J. Slevin, "Radio Pulses from Extensive Air Showers at 520 MHz," Nature 217, 440 (1968).

[41] D. J. Fegan and D. M. Jennings, "UHF Radio Pulses from the Zenith Associated with Extensive Air Showers," Nature 223, 722 (1969).

[42] Auger references, ICRCs 2005, 2007: arXiv:0707.2638.

[43] Lythe, M. B., D. G. Vaughan, and the BEDMAP Consortium (2001), BEDMAP: A new ice thickness and subglacial topographic model of Antarctica, J. Geophys. Res., 106(B6), 11,33511,351

[44] Bassin, C., Laske, G. and Masters, G., The Current Limits of Resolution for Surface Wave Tomography in North America, EOS Trans AGU, 81, F897, 2000.

[45] Ghandhi,Reno,Quigg,Sarcevic, hep-ph/9512364.

[46] SunCat Solar, LLC, 9121 E. Tanque Verde Rd., Tucson AZ 85749.

[47] http://www.pol.ac.uk/psmsl/puscience/

[48] H. Nyquist, "Thermal Agitation of Electric Charge in Conductors,," Phys. Rev. 32, 110, (1928).

[49] J. Alvarez-Muniz, Phys.Lett.B411:218-224 (1997) astro-ph/9706064.

[50] J. Alvarez-Muniz, Phys.Lett.B434:396 (1998) astro-ph/9806098.

[51] J. Alvarez-Muniz, et al., Phys. Rev. D 74, 023007 (2006) astro-ph/0512337.

[52] D.Z. Besson et al. (for the ANITA collaboration) "In situ radioglaciological measurements near Taylor Dome, Antarctica and implications for ultra-high energy (UHE) neutrino astronomy" Astropart. Phys., Volume 29, Issue 2, March 2008, 130-157

[53] M. Nieto-Vesperinas et al., "Light transmission from a randomly rough dielectric diffuser: theoretical and experimental results," Optics Letters 15, 1261 (1990).

[54] T. Furukawa, K. Kamiyama and H. Maeno, "Snow Surface Features along the Traverse Route from the Coast to Dome Fuji Station, Queen Maud Land, Antarctica," Proc. NIPR Symp. Polar Meteorol. Glaciol., 10, 13-24 (1996).

[55] I. Goodwin, "Snow Accumulation and Surface Topology in the Katabatic Zone of Eastern Wilkes Land, Antarctica", Antarctic Science 2, 3, 235-242 (1990).

[56] M. Griswold and D. Saltzberg, "Review of Literature Regarding Small-Scale Surface Roughness in Antarctica" ANITA-note \#108 http://www.phys.hawaii.edu:8080/anita_notes/108.

[57] A. Romero-Wolf and D. Saltzberg, "Taylor Dome Snow Surface Roughness," ANITA-note \#330. http://www.phys.hawaii.edu:8080/anita_notes/330.

[58] M. Griswold, M. Harrison and D. Saltzberg, "Observation of Light Transmission Through Random Rough Glass Surfaces Beyond the critical Angle, J. Op. Soc. Am. A. 24, 3207 (2007); More detail is given in the following: B. Daub et al. ANITA-note \#77, http://www.phys.hawaii.edu:8080/anita_notes/77 and M. Harrison et al. ANITA-note \#101, http://www.phys.hawaii.edu:8080/anita_notes/101.

[59] A. Dogariu and G. Boreman, "Facet Model for Photon-Flux Transmission through Rough Dielectric Interfaces", Optics Letters 21, 701 (1996).

[60] Dookayka, K., Ph.D. dissertation, in preparation, University of California, Irvine (2009).

[61] $P_{N}=k_{B} T_{s y s} \Delta f$, for $k_{B}=1.38 \times 10^{-23} \mathrm{~J} / \mathrm{K}, T_{s y s}=$ the system noise temperature in Kelvin, and $\Delta f=$ the bandwidth in Hz. 